\title{
Irreducible Modules of Quantized Enveloping Algebras at Roots of 1
}

\author{
By \\ Nanhua $\mathrm{XI}^{*}$ \\ Introduction
}

Let $\mathbf{A}$ be an associative algebra over a field. An interesting problem is to understand the structure of irreducible modules of $\mathbf{A}$ (of finite dimensions). More or less, this is equivalent to understand the structure of maximal left ideals of $\mathbf{A}$ (of finite codimensions). For the latter, it would be helpful if we know the generators of the maximal left ideals.

In Lie theory, there are some infinite dimensional algebras associated to a semisimple Lie algebra $\mathfrak{g}$ over $\mathbf{C}$. We shall be only concerned with the following four of them.

(i) The universal enveloping algebra $\mathfrak{U}$ of $\mathfrak{g}$.

(ii) The hyperalgebra $\mathfrak{U}_{\mathfrak{f}}:=\mathfrak{U}_{\mathbf{z}} \otimes_{\mathbf{z}} \mathfrak{f}$, where $\mathfrak{U}_{\mathbf{z}}$ is the Kostant $\mathbf{Z}$-form of $\mathfrak{U}$ and $\mathfrak{f}$ is an algebraically closed field of prime characteristic.

(iii) The quantized enveloping algebra $U$ (over $\mathbf{Q}(v), v$ is an indeterminate) of $\mathfrak{g}$.

(iv) The quantized hyperalgebra $U_{\xi}:=U_{\mathbf{Q}\left[v, v^{-1}\right]} \otimes_{\mathbf{Q}\left[v, v^{-1}\right]} \mathbf{Q}(\xi)$, where $\xi \in \mathbf{C}^{*}$ and $U_{\mathbf{Q}\left[v, v^{-1}\right]}$ is a $\mathbf{Q}\left[v, v^{-1}\right]$-form of $U$ [L1, Section 4.1, p. 243], and $\mathbf{Q}(\xi)$ is regarded as a $\mathbf{Q}\left[v, v^{-1}\right]$-algebra through the $\mathbf{Q}$-algebra homomorphism $\mathbf{Q}\left[v, v^{-1}\right] \rightarrow \mathbf{Q}(\xi), v \rightarrow \xi$.

We are mainly interested in finite dimensional irreducible modules of these algebras, or equivalently, in maximal left ideals of the algebras of finite codimensions. The generators of maximal left ideals of $\mathfrak{U}$ with finite codimensions are known more than forty years ago [HC, Lemma 15, p.42]. Thanks to the works [L1, Theorem 4.12, p. 247] and [APW, Corollary 7.7, p. 40], a similar result holds for maximal left ideals of $U$ and of $U_{\xi}$ with finite codimensions provided that $\xi$ is not a root of 1 or $\xi^{2}=1$. We will review these results in Section 1.2.

The purpose of the paper is to find out the counterparts of the above

Communicated by M. Kashiwara, March 13, 1995. Revised August 11, 1995.

1991 Mathematics Subject Classification(s): 17B37, 17B10.

* Institute of Mathematics, Academia Sinica, Beijing 100080, China. 
results for the hyperalgebra $\mathfrak{U}_{\mathfrak{f}}$ and for the quantized hyperalgebra $U_{\xi}$ when $\xi$ is a root of 1 of order $\geq 3$. The main results might lead to a way to compute the characters of finite dimensional irreducible modules of $\mathfrak{U}_{\mathfrak{i}}$ and of $U_{\xi}$.

The basic idea is simple. When $\xi$ is a root of 1 of order $\geq 3$, the algebra $U_{\xi}$ has a Frobenius kernel $\mathbf{u}_{\xi}$ [L4, Theorem 8.3, p. 107]. The Frobenius kernel $\mathbf{u}_{\xi}$ is a $\mathbf{Q}(\xi)$-algebra of finite dimension. Moreover, the algebra $\mathbf{u}_{\xi}$ has a triangular decomposition $\mathbf{u}_{\xi}=\mathbf{u}_{\xi}^{-} \mathbf{u}_{\xi}^{0} \mathbf{u}_{\xi}^{+}$. Each Verma module of $\mathbf{u}_{\xi}$ has a unique irreducible submodule, and each irreducible $\mathbf{a}_{\xi}$-module $L$ is an irreducible submodule of certain Verma module $Z$ of $\mathbf{u}_{\xi}$. As a $\mathbf{a}_{\xi}^{-}$-modules, $Z$ is isomorphic to $\mathbf{u}_{\xi}^{-}$. Therefore there exists an element $x$ in $\mathbf{u}_{\xi}^{-}$such that as $\mathbf{u}_{\xi}^{-}$-modules $L$ is isomorphic to $\mathbf{u}_{\xi}^{-} x$. It turns out that the element $x$ is a monomial of the generators of $U_{\xi}^{-}$(the negative part of $U_{\xi}$ ). So the generators of the maximal left ideal of $\mathbf{u}_{\xi}$ corresponding to $L$ can be described explicitly (Theorem 5.3). But $L$ is a restriction to $\mathbf{u}_{\xi}$ of certain irreducible $U_{\xi}$-module [L2, Prop. 7.1 (c), p. 70]. Using tensor product theorem [L2, Theorem 7.4, p.73], we can give the generators of maximal left ideals of $U_{\xi}$ of finite codimensions (Theorem 5.4). The same idea is valid to the hyperalgebra $\mathfrak{U}_{\mathfrak{f}}$.

The paper is organized as follows. In Section 1 we recall some basic definitions and review some results in [APW, HC, L1-L4]. In Section 2 we consider the Frobenius kernel $\mathbf{u}_{\xi}$. In Section 3 we consider the category of finite dimensional $U_{\xi}$-modules of type 1 . In Section 4 we prove that certain monomials in $U_{\xi}^{-}$are actually in $\mathbf{u}_{\xi}^{-}$. For a technical reason we require that every simple component of $g$ is not of type $G_{2}$. In Section 5 we give the main theorems of the paper. In Section 6 we consider the hyperalgebra $\mathfrak{U}_{\mathfrak{f}}$. In Section 7 we give some questions.

\section{Acknowledgement}

I would like to express my hearty thanks to H.H. Andersen, F. Hirzebruch, G. Lusztig and B. Srinivasan for their kind encouragements. I wish to thank G. Lusztig for helpful comments.

I am grateful to Max-Planck-Institut für Mathematik for financial support and hospitality. Part of the work was done during my visit at IHES, Bures-sur-Yvette, 1993 Fall. I am grateful to IHES for financial support and hospitality.

I am very grateful to the referee for helpful comments.

\section{§1. Quantized Hyperalgebra}

1.1. Let $\mathfrak{g}$ be a semisimple Lie algebra over $\mathbb{C}$ with rank $n$ and let $\left(a_{i j}\right)$ be the Cartan matrix associated to $g$. We can find integers $d_{i}$ in $\{1,2,3\}$ such 
that $\left(d_{i} a_{i j}\right)$ is a symmetric matrix. Assume that the sum of all $d_{i}$ is as small as possible.

Let $U$ be the quantized enveloping algebra of $\mathfrak{g}$ over $\mathbf{Q}(v)$ with parameter $v$ ( $v$ an indeterminate). By definition, $U$ is an associative $\mathbf{Q}(v)$-algebra and has generators $E_{i}, F_{i}, K_{i}, K_{i}^{-1}(i=1,2, \ldots, n)$, which satisfy certain relations (see for example, $[\mathrm{L} 4,1.1, \mathrm{p} .90]$ ). The algebra $U$ is in fact a Hopf algebra, the coproduct $\Delta$, antipode $S$, counit $\varepsilon$ are defined as follows:

$$
\begin{gathered}
\Delta\left(E_{i}\right)=E_{i} \otimes 1+K_{i} \otimes E_{i}, \quad \Delta\left(F_{i}\right)=F_{i} \otimes K_{i}^{-1}+1 \otimes F_{i}, \quad \Delta\left(K_{i}\right)=K_{i} \otimes K_{i}, \\
S\left(E_{i}\right)=-K_{i}^{-1} E_{i}, \quad S\left(F_{i}\right)=-F_{i} K_{i}, \quad S\left(K_{i}\right)=K_{i}^{-1}, \\
\varepsilon\left(E_{i}\right)=\varepsilon\left(F_{i}\right)=0, \quad \varepsilon\left(K_{i}\right)=1 .
\end{gathered}
$$

We need some notations to introduce quantized hyperalgebras and for later uses. Given an integer $a$ and positive integers $b, d$, set

$$
\begin{gathered}
{[a]_{d}:=\frac{v^{a d}-v^{-a d}}{v^{d}-v^{-d}}, \quad[b]_{d}^{!}:=\prod_{h=1}^{b} \frac{v^{h d}-v^{-h d}}{v^{d}-v^{-d}}, \quad[0]_{d}^{!}:=1, \quad[-b]_{d}^{!}=(-1)^{b}[b]_{d}^{!} ;} \\
{\left[\begin{array}{l}
a \\
b
\end{array}\right]_{d}:=\prod_{h=1}^{b} \frac{v^{(a-h+1) d}-v^{-(a-h+1) d}}{v^{h d}-v^{-h d}}, \quad\left[\begin{array}{c}
a \\
0
\end{array}\right]_{d}:=1, \quad\left[\begin{array}{c}
a \\
-b
\end{array}\right]_{d}:=0 .}
\end{gathered}
$$

Note that $\left[\begin{array}{l}a \\ b\end{array}\right]_{d}$ is in $\mathbf{Q}\left[v, v^{-1}\right]$, we shall denote $\left[\begin{array}{l}a \\ b\end{array}\right]_{d, \xi}$ the evaluation of $\left[\begin{array}{l}a \\ b\end{array}\right]_{d}$ at $\xi$ for any $\xi$ in $\mathbf{C}^{*} \cup\{v\}$. Of course, we have $\left[\begin{array}{l}a \\ b\end{array}\right]_{d, v}=\left[\begin{array}{l}a \\ b\end{array}\right]_{d}$.

The quantized hyperalgebra $U_{\xi}\left(\xi \in \mathbf{C}^{*}\right)$ is defined as follows. Let $U_{\mathbf{Q}\left[v, v^{-1}\right]}$ be the $\mathbf{Q}\left[v, v^{-1}\right]$-subalgebra of $U$ generated by the elements $E_{i}^{(a)}:=E_{i}^{a} /[a]_{d_{i}}^{!}$, $F_{i}^{(a)}:=F_{i}^{a} /[a]_{d_{i}}^{!}, K_{i}, K_{i}^{-1}$ for $i=1,2, \ldots, n, a \geq 0$. Regard $\mathbf{Q}(\xi)$ as a $\mathbf{Q}\left[v, v^{-1}\right]-$ algebra through the $\mathbf{Q}$-algebra homomorphism $\mathbf{Q}\left[v, v^{-1}\right] \rightarrow \mathbf{Q}(\xi), v \rightarrow \xi$. Define $U_{\xi}:=U_{\mathbf{Q}\left[v, v^{-1}\right]} \otimes_{\mathbf{Q}\left[v, v^{-1}\right]} \mathbf{Q}(\xi)$ and call $U_{\xi}$ a quantized hyperalgebra (associated to $\left(a_{i j}\right)$ with parameter $\xi$ ). For convenience, set $U_{v}:=U$. The algebra $U_{\xi}$ inherits a Hopf algebra structure from that of $U_{\mathbf{Q}\left[v, v^{-1}\right]}$, denote again by $\Delta$ the coproduct, by $S$ the antipode and by $\varepsilon$ the counit. The tensor product of two $U_{\xi}$-modules then has a natural $U_{\xi}$-module structure by means of the coproduct, and the antipode can be used to define the dual module of a $U_{\xi}$-module.

For an integer $c$ and a positive integer $a$ we set

$$
\left[\begin{array}{c}
K_{i}, c \\
a
\end{array}\right]:=\prod_{h=1}^{a} \frac{K_{i} v^{(c-h+1) d_{l}}-K_{i}^{-1} v^{-(c-h+1) d_{2}}}{v^{h d_{2}}-v^{-h d_{i}}} \text { and }\left[\begin{array}{c}
K_{i}, c \\
0
\end{array}\right]:=1 .
$$

We have $\left[\begin{array}{c}K_{i}, c \\ a\end{array}\right] \in U_{\mathbf{Q}\left[v, v^{-1}\right]}[\mathrm{L} 1$, Lemma 4.4, p. 244]. For simplicity, the 
images in $U_{\xi}$ of $E_{i}^{(a)}, F_{i}^{(a)}, K_{i}, K_{i}^{-1},\left[\begin{array}{c}K_{i}, c \\ a\end{array}\right]$, etc. will be denoted by the same notations respectively. For convenience, we set $E_{i}^{(a)}:=0, F_{i}^{(a)}:=0$ for all $i$ and $a<0$.

The algebra $U_{\xi}$ has a triangular decomposition. Let $U_{\xi}^{+}\left(\right.$resp. $\left.U_{\xi}^{-} ; U_{\xi}^{0}\right)$ be the subalgerba of $U_{\xi}$ generated by the elements $E_{i}^{(a)}\left(\operatorname{resp} . F_{i}^{(a)} ; K_{i}, K_{i}^{-1}\right.$, $\left.\left[\begin{array}{c}K_{i}, c \\ a\end{array}\right], c \in \mathbb{Z}\right)$ for $i=1,2, \ldots, n, a \geq 0$. The multiplication in $U_{\xi}$ defines a $\mathbf{Q}(\xi)$-space isomorphism between $U_{\xi}^{-} \otimes U_{\xi}^{0} \otimes U_{\xi}^{+}$and $U_{\xi}$.

1.2. Given $\lambda=\left(\lambda_{1}, \lambda_{2}, \ldots, \lambda_{n}\right) \in \mathbb{Z}_{+}^{n}, \sigma=\left(\sigma_{1}, \sigma_{2}, \ldots, \sigma_{n}\right) \in\{ \pm 1\}^{n}$, let $I_{\lambda, \sigma}^{+}$be the left ideal of $U_{\xi}$ generated by the elements $E_{i}^{(a)}, K_{i}-\sigma_{i} \xi^{\lambda_{2} d_{2}},\left[\begin{array}{c}K_{i}, c \\ a\end{array}\right]-$ $\sigma_{i}^{a}\left[\begin{array}{c}\lambda_{i}+c \\ a\end{array}\right]_{d_{i}, \xi}$ for $i=1,2, \ldots, n, a \geq 1, c \in \mathbf{Z}$, and let $I_{\lambda}^{-}$be the left ideal of $U_{\xi}^{-}$generated by the elements $F_{i}^{\left(a_{l}\right)}$ for all $i$ and $a_{i} \geq \lambda_{i}+1$. Then let $I_{\lambda, \sigma}$ be the left ideal of $U_{\xi}$ generated by all elements in $I_{\lambda, \sigma}^{+} \cup I_{\lambda}^{-}$. Then

(i) The $U_{\xi}$-module $V_{\xi}(\lambda, \sigma):=U_{\xi} / I_{\lambda, \sigma}$ is of finite dimension and has a unique irreducible quotient module, denoted by $L_{\xi}(\lambda, \sigma)$. The dimension of $V_{\xi}(\lambda, \sigma)$ is given by Weyl's character formula. [L1, Theorem 4.12, p. 247]. We shall denote $v_{\lambda, \sigma}$ the image in $V_{\xi}(\lambda, \sigma)$ of the neutral element $1 \in U_{\xi}$, and denote $\bar{v}_{\lambda, \sigma}$ the image in $L_{\xi}(\lambda, \sigma)$ of $v_{\lambda, \sigma}$.

Sometimes we call $V_{\xi}(\lambda, \sigma)$ a Weyl module of $U_{\xi}$.

(ii) The map $(\lambda, \sigma) \rightarrow L_{\xi}(\lambda, \sigma)$ defines a bijection between the set $\mathbb{Z}_{+}^{n} \times\{ \pm 1\}^{n}$ and the set of isomorphism classes of irreducible $U_{\xi}$-modules of finite dimensions. [L1, Prop. 2.6 and Prop. 3.2, p. 241] and [L2, Prop. 6.4, p. 69].

(iii) One has

$$
V_{\xi}(\lambda, \sigma) \simeq V_{\xi}(\lambda, \mathbb{1}) \otimes \mathbf{Q}(\xi)_{\sigma}, \quad L_{\xi}(\lambda, \sigma) \simeq L_{\xi}(\lambda, \mathbb{1}) \otimes \mathbf{Q}(\xi)_{\sigma},
$$

where $\mathbb{1}=(1,1, \ldots, 1) \in\{ \pm 1\}^{n}$ and $\mathbf{Q}(\xi)_{\sigma}$ is the one dimensional $U_{\xi}$-module on which all $E_{i}^{(a)}, F_{i}^{(a)}(i=1,2, \ldots, n, a \geq 1)$ act by scalar zero and $K_{i},\left[\begin{array}{c}K_{i}, c \\ a\end{array}\right]$ $(i=1,2, \ldots, n, c \in \mathbb{Z}, a \in \mathbf{N})$ act by scalar $\sigma_{i}, \sigma_{i}^{a}\left[\begin{array}{l}c \\ a\end{array}\right]_{d_{i}, \xi}$ respectively. [APW,
1.6 , pp. 6-7]. (iv) Provided that $\xi$ is not a root of 1 or $\xi^{2}=1$, then $V_{\xi}(\lambda, \sigma)$ is irreducible, i.e. $V_{\xi}(\lambda, \sigma) \simeq L_{\xi}(\lambda, \sigma)$. And every finite dimensional $U_{\xi}$-module is completely reducible. [L4, 7.2, pp. 105-106; APW, Corollary 7.7, p. 40].

Therefore, the theory of finite dimensional $U_{\xi}$-modules is well understood when $\xi$ is not a root of 1 or $\xi^{2}=1$. When $\xi$ is a root of 1 of order $\geq 3$ we do not know much about the irreducible module $L_{\xi}(\lambda, \sigma)$. In Section 5 
we shall describe the generators of the maximal left ideal $J_{\lambda, \sigma}$ of $U_{\xi}$ corresponding to $L_{\xi}(\lambda, \sigma)$. To have a look what the generators are we introduce some monomials of $F_{i}^{(a)}(i=1,2, \ldots, n, a \geq 0)$. These monomials play a central role in the paper.

1.3. Set $\alpha_{i}=\left(a_{1 i}, a_{2 i}, \ldots, a_{n i}\right) \in \mathbf{Z}^{n}$. For every $\mu=\left(\mu_{1}, \mu_{2}, \ldots, \mu_{n}\right) \in \mathbf{Z}^{n}$, we also write $\left\langle\mu, \alpha_{i}^{v}\right\rangle$ for $\mu_{i}$. Define $s_{i}: \mathbf{Z}^{n} \rightarrow \mathbf{Z}^{n}$ by $s_{i} \mu=\mu-\left\langle\mu, \alpha_{i}^{v}\right\rangle \alpha_{i}$. The reflections $s_{1}, s_{2}, \ldots, s_{n}$ generate the Weyl group $W$ of the Cartan matrix $\left(a_{i j}\right)$.

Let $\lambda=\left(\lambda_{1}, \lambda_{2}, \ldots, \lambda_{n}\right) \in \mathbf{Z}_{+}^{n}$. Assume that $s_{i_{1}} s_{i_{2}} \cdots s_{i_{k}}$ is a reduced expression of an element $w$ in $W$. Set $\lambda_{\mathbf{i}, 1}=\lambda_{i_{1}}, \lambda_{\mathbf{i}, 2}=\left\langle s_{i_{1}} \lambda, \alpha_{i_{2}}^{v}\right\rangle, \ldots, \lambda_{\mathbf{i}, k}=\left\langle s_{i_{k-1}} \ldots\right.$ $\left.s_{i_{1}} \lambda, \alpha_{i_{k}}^{v}\right\rangle$, where $\mathbf{i}=\left(i_{1}, i_{2}, \ldots, i_{k}\right)$. Define

$$
x_{\lambda, w, \mathbf{i}}:=F_{i_{1}}^{\left(\lambda_{\mathbf{i}, 1}\right)} F_{i_{2}}^{\left(\lambda_{\mathbf{i}, 2)}\right)} \cdots F_{i_{k}}^{\left(\lambda_{\mathbf{i}, k}\right)}, \quad \text { and } \quad x_{\lambda, w^{-1}, \mathbf{i}}^{\prime}:=F_{i_{k}}^{\left(\lambda_{\mathbf{i}, k)}\right)} F_{i_{k-1}}^{\left(\lambda_{\mathbf{i}, k-1}\right)} \cdots F_{i_{1}}^{\left(\lambda_{\mathbf{i}, 1}\right)} .
$$

Depending on the contexts, the monomials will be regarded as elements in $U_{\xi}\left(\xi \in \mathbf{C}^{*}\right)$ or elements in $U$. Note that in the universal enveloping algebra $\mathfrak{U}$ of $\mathfrak{g}$ similar elements are defined by Verma [V, Theorem 4, p. 162].

Lemma 1.4. The elements $x_{\lambda, w, \mathbf{i}}$ and $x_{\lambda, w^{-1, i}}^{\prime}$ are independent of the choice of the reduced expression of $w$, only depend on $\lambda$ and $w$. We shall denote them $x_{\lambda, w}$ and $x_{\lambda, w^{-1}}^{\prime}$ respectively. When $w$ is the longest element $w_{0}$ of $W$, we simply write $x_{\lambda}$ and $x_{\lambda}^{\prime}$ for $x_{\lambda, w}$ and $x_{\lambda, w}^{\prime}$ respectively.

Proof. Use the quantum Verma identity [L7, Prop. 39.3.7, p. 313].

1.5. From now on $\xi$ will be a root of 1 with order $l \geq 3$. Let $l_{i}$ be the order of $\xi^{2 d_{i}}$ and set $\kappa:=\left(l_{1}-1, l_{2}-1, \ldots, l_{n}-1\right)$. We say that an element $\lambda=\left(\lambda_{1}, \lambda_{2}, \ldots, \lambda_{n}\right) \in \mathbf{Z}_{+}^{n}$ is $\mathbf{l}$-restricted if $\lambda_{1} \leq l_{1}-1, \ldots, \lambda_{n} \leq l_{n}-1$. For each $\mu=\left(\mu_{1}, \mu_{2}, \ldots, \mu_{n}\right) \in \mathbf{Z}^{n}$ we set $\mathbf{l} \mu:=\left(l_{1} \mu_{1}, l_{2} \mu_{2}, \ldots, l_{n} \mu_{n}\right)$.

Let $\lambda, \mu \in \mathbf{Z}_{+}^{n}, \sigma \in\{ \pm 1\}^{n}$ and assume that $\lambda$ is l-restricted. Let $J_{\mathbf{1} \mu+\lambda, \sigma}$ be the left ideal of $U_{\xi}$ generated by all elements in $I_{1 \mu+\lambda, \sigma}$ and elements $F$ in $U_{\xi}^{-}$such that $F x_{\kappa-\lambda}=0$, one main result of the paper says that $U_{\xi} / J_{1 \mu+\lambda, \sigma} \simeq$ $L_{\xi}(1 \mu+\lambda, \sigma)$ (Theorem 5.4) provided that every indecomposable component of the Cartan matrix $\left(a_{i j}\right)$ is not of type $G_{2}$. One key step to reach the result is the assertion that $x_{\kappa+w_{0} \lambda}^{\prime}\left(=x_{\kappa-\lambda}\right)$ belongs to the Frobenius kernel (Theorem 4.2).

1.6. Remark. Some results in [L1-L4] are stated and proved in full generality in [L7]. The other results in [L1-L4] can be stated and proved in full generality along the same ways in [L1-L4]. Therefore the author feels free to quote the results in [L1-L4] in full generality forms.

\section{§2. Frobenius Kernel}

2.1. Recall that $\xi$ is a root of 1 with order $l \geq 3$ and $l_{i}$ is the order of $\xi^{2 d_{2}}$. Let $R^{+}$be the set of positive roots of the root system $R:=W\left\{\alpha_{1}, \alpha_{2}, \ldots\right.$, 
$\left.\alpha_{n}\right\} \subset \mathbb{Z}^{n}$. Set $l_{\alpha}:=l_{i}, d_{\alpha}:=d_{i}$ if $\alpha=w\left(\alpha_{i}\right)$ for some $w$ in $W$. For each positive root $\alpha$ in $R^{+}$, let $E_{\alpha}, F_{\alpha}$ be the root vectors defined in [L4, Theorem 6.6 (iii), p. 104]. For a non-negative integer $a$, we also write $E_{\alpha}^{(a)}, F_{\alpha}^{(a)}$ for $E_{\alpha}^{a} /[a]_{d_{\alpha}}^{!}$, $F_{\alpha}^{a} /[a]_{d_{\alpha}}^{!}$respectively.

Let $U_{\xi, l}$ be the subalgebra of $U_{\xi}$ generated by the elements $E_{i}^{\left(a l_{2}\right)}, F_{i}^{\left(a l_{l}\right)}$, $K_{i}^{ \pm l_{2}},\left[\begin{array}{c}K_{i}, c \\ a l_{i}\end{array}\right]$ for $i=1,2, \ldots, n, c \in \mathbb{Z}, a \in \mathbb{N}$. The positive part $U_{\xi, l}^{+}$, the negative part $U_{\xi, l}^{-}$and the zero part $U_{\xi, l}^{0}$ of $U_{\xi, l}$ are defined in an obvious way. Let $\mathbf{u}_{\xi}$ be the subalgebra of $U_{\xi}$ generated by the elements $E_{\alpha}, F_{\alpha}, K_{i}$, $K_{i}^{-1}$ for $\alpha \in R_{l}^{+}:=\left\{\alpha \in R^{+} \mid l_{\alpha} \geq 2\right\}$ and $i=1,2, \ldots, n$. The algebra is called the Frobenius kernel of $U_{\xi}$. The Frobenius kernel $\mathbf{u}_{\xi}$ is a Hopf algebra and $\operatorname{dim}_{\mathbf{Q}(\xi)} \mathbf{u}_{\xi}=2^{n} \prod_{i=1}^{n} l_{i} \prod_{\alpha \in R^{+}} l_{\alpha}^{2}[\mathrm{~L} 4,8.11$, p. 111, and Theorem 8.3, p. 107]. We define the positive part $\mathbf{u}_{\xi}^{+}$, the negative part $\mathbf{u}_{\xi}^{-}$and the zero part $\mathbf{u}_{\xi}^{0}$ of $\mathbf{u}_{\xi}$ in an obvious manner.

2.2. The following are some properties concerned with the algebras $U_{\xi, l}$ and $\mathbf{u}_{\xi}$, which are due to Lusztig.

(i) Assume that $\left(a_{i j}\right)$ is indecomposable. Then there exists a unique $\mathbf{Q}(\xi)$-algebra homomorphism $U_{\xi, l} \rightarrow U_{\xi^{*}}^{*} \otimes_{\mathbf{Q}} \mathbf{Q}(\xi)$ such that $E_{i}^{\left(a l_{l}\right)} \rightarrow E_{i}^{(a)}, F_{i}^{\left(a l_{2}\right)} \rightarrow$ $F_{i}^{(a)}, K_{i}^{ \pm l_{2}} \rightarrow K_{i}^{ \pm 1},\left[\begin{array}{c}K_{i}, 0 \\ a l_{i}\end{array}\right] \rightarrow\left[\begin{array}{c}K_{i}, 0 \\ a\end{array}\right]$, and $E_{i}^{\left(b_{t}\right)} \rightarrow 0, F_{i}^{\left(b_{t}\right)} \rightarrow 0,\left[\begin{array}{c}K_{i}, 0 \\ b_{i}\end{array}\right] \rightarrow 0$, for $i=1,2, \ldots, n, a \in \mathbf{N}, b_{i} \in \mathbf{N}-l_{i} \mathbf{N}$, where $U_{\xi^{*}}^{*}=U_{\xi^{*}}$ when $l_{1}=l_{2}=\cdots=l_{n}$ and $U_{\xi^{*}}^{*}$ is the quantized hyperalgebra associated to the transpose matrix of $\left(a_{i j}\right)$ with parameter $\xi^{*}$ when $l_{k}=d_{m} l_{m}$ for some $k, m$ with $1=d_{k}<d_{m} \in\{2,3\}$, and $\xi^{*}=\xi^{l_{1}^{2}}$ when $l_{1}=l_{2}=\cdots=l_{n}$ and $\xi^{*}=\xi^{d_{m} l_{m}^{2}}$ when $l_{k}=d_{m} l_{m}$ for some $k, m$ with $1=d_{k}<d_{m} \in\{2,3\}$. (Note that $l_{i}=l_{j}$ if $\alpha_{i}, \alpha_{j}$ are conjugate under $W$. So $\xi^{*}$ does not depend on the choice of $m$ and is well defined.) [L7, Theorems 35.1 .9 , p. $270 ; 35.5 .2$, p. 279; L4, Theorem 8.10, p. 110].

We always have $\xi^{*}= \pm 1$. Actually, if $l_{1}=l_{2}=\cdots=l_{n}$, choose $i$ such that $d_{i}=1$, then $\xi^{*}=\xi^{l^{2}}=( \pm 1)^{l_{l}}= \pm 1$, if $l_{k}=d_{m} l_{m}$ for some $k, m$ with $1=d_{k}<d_{m} \in\{2,3\}$, then $\xi^{*}=\xi^{d_{m} l_{m}^{2}}=( \pm 1)^{l_{m}}= \pm 1$.

(ii) Let $\left\{x_{a}\right\}$ be a $\mathbf{Q}(\xi)$-basis of $\mathbf{u}_{\xi}^{-}$and $\left\{y_{b}\right\}$ be a $\mathbf{Q}(\xi)$-basis of $U_{\xi, l}^{-}$, then $\left\{x_{a} y_{b}\right\}$ is a $\mathbf{Q}(\xi)$-basis of $U_{\xi}^{-}$, so is $\left\{y_{b} x_{a}\right\}$ [L4, Lemma 8.8, p. 109; L7, Theorem 35.4.2 (b), p. 276, 35.5.2, p. 279].

(iii) The elements $\prod_{\alpha \in R_{l}^{+}} F_{\alpha}^{\left(a_{\alpha}\right)} \prod_{i=1}^{n} K_{i}^{b_{i}} \prod_{\alpha \in R_{l}^{+}} E_{\alpha}^{\left(a_{\alpha}^{\prime}\right)}\left(0 \leq a_{\alpha}, a_{\alpha}^{\prime} \leq l_{\alpha}-1,0 \leq b_{i} \leq\right.$ $\left.2 l_{i}-1\right)$ form a $\mathbf{Q}(\xi)$-basis of $\mathbf{u}_{\xi}$; the elements $\prod_{\alpha \in R_{l}^{+}} F_{\alpha}^{\left(a_{\alpha}\right)}\left(0 \leq a_{\alpha} \leq l_{\alpha}-1\right)$ form a $\mathbf{Q}(\xi)$-basis of $\mathbf{u}_{\xi}^{-}$; the elements $\prod_{i=1}^{n} K_{i}^{b_{1}}\left(0 \leq b_{i} \leq 2 l_{i}-1\right)$ form a $\mathbf{Q}(\xi)$-basis of $\mathbf{u}_{\xi}^{0}$; the elements $\prod_{\alpha \in R_{l}^{+}} E_{\alpha}^{\left(a_{\alpha}^{\prime}\right)}\left(0 \leq a_{\alpha}^{\prime} \leq l_{\alpha}-1\right)$ form a $\mathbb{Q}(\xi)$-basis of $\mathbf{u}_{\xi}^{+}$. [L4, Theorem 8.3, p. 107].

(iv) Let $\lambda, \mu \in \mathbb{Z}_{+}^{n}$ and $\sigma \in\{ \pm 1\}^{n}$. Assume that $\lambda$ is l-restricted. Then [L2, Theorem 7.4, p. 73] 


$$
L_{\xi}(\mathbf{l} \mu+\lambda, \sigma) \simeq L_{\xi}(\mathbf{l} \mu, \sigma) \otimes L_{\xi}(\lambda, \mathbf{1}) \simeq L_{\xi}(\mathbf{l} \mu, \mathbf{1}) \otimes L_{\xi}(\lambda, \sigma) .
$$

(v) Assume that $\left(a_{i j}\right)$ is indecomposable. The restriction to $U_{\xi, l}$ of $L_{\xi}(1 \mu, \sigma)$ is an irreducible $U_{\xi, l}$-module, and $E_{\alpha} L_{\xi}(\mathbf{l} \mu, \sigma)=F_{\alpha}\left(L_{\xi}(\mathbf{l} \mu, \sigma)=0\right.$ for all $\alpha \in R_{l}^{+}$. By this and (i) we see there is a $U_{\xi^{*}}^{*} \otimes_{\mathbf{Q}} \mathbf{Q}(\xi)$-module structure on $L_{\xi}(l \mu, \sigma)$ which is compatible with the homomorphism in (i). Moreover, $L_{\xi}(\mathbf{l} \mu, \sigma)$ is an irreducible $U_{\xi^{*}}^{*} \otimes_{\mathbf{Q}} \mathbf{Q}(\xi)$-module corresponding to $\left(\mu, \sigma^{\prime}\right)$ for a suitable $\sigma^{\prime} \in\{ \pm 1\}^{n}$. So dimension of $L_{\xi}(\mathbf{l} \mu, \sigma)$ can be computed through Weyl's character formula. [L7, Prop. 35.3.2, p. 273, Corollary 35.3.4, p. 275; L2, Prop. 7.5 (b), p. 74].

(vi) As a $\mathbf{u}_{\xi}$-module, $L_{\xi}(\lambda, \sigma)$ is irreducible if $\lambda$ is $l$-restricted. The map $(\lambda, \sigma) \rightarrow L_{\xi}(\lambda, \sigma)$ defines a bijections between the set $\mathbf{Z}_{+, 1}^{n} \times\{ \pm 1\}^{n}$ and the set of isomorphism classes of irreducible $\mathbf{u}_{\xi}$-modules, where $\mathbf{Z}_{+, 1}^{n}$ is the set of all l-restricted elements in $\mathbf{Z}_{+}^{n}$ [L3, Prop. 5.11, p. 291].

According to (i-vi), the algebra $\mathbf{u}_{\xi}$ is a key to understand $U_{\xi}$. For convenience, we consider the subalgebra $\tilde{\mathbf{u}}_{\xi}$ of $U_{\xi}$ generated by all elements in $\mathbf{u}_{\xi} \cup U_{\xi}^{0}$. One has $\tilde{\mathbf{u}}_{\xi}=\mathbf{u}_{\xi}^{-} U_{\xi}^{0} \mathbf{u}_{\xi}^{+}$. By (vi) we see

(vii) Assume that $(\lambda, \sigma) \in \mathbf{Z}_{+1}^{n} \times\{ \pm 1\}^{n}$, then the restriction to $\tilde{\mathbf{u}}_{\xi}$ of the irreducible $U_{\xi}$-module $L_{\xi}(\lambda, \sigma)$ is an irreducible $\tilde{\mathbf{u}}_{\xi}$-module, denoted by $\widetilde{L}_{\xi}(\lambda, \sigma)$.

2.3. To go further we need some notions. Let $\gamma \in \mathbf{Z R}$. An element $x$ in $U_{\xi}$ is said to have degree $\gamma$ if $K_{i} x K_{i}^{-1}=\xi^{-\left\langle\gamma, \alpha_{v}^{v}\right\rangle d_{2}} x$ and $\left[\begin{array}{c}K_{i}, c \\ a\end{array}\right] x=$ $x\left[\begin{array}{c}K_{i}, c-\left\langle\gamma, \alpha_{i}^{\vee}\right\rangle \\ a\end{array}\right]$ for $i=1,2, \ldots, n, \quad c \in \mathbf{Z}, \quad a \in \mathbf{N}$. We also call $x$ a homogenous element (of degree $\gamma$ ) and write $\operatorname{deg}(x)=\gamma$.

Let $U_{\xi}^{\prime}$ be a subalgebra of $U_{\xi}$ containing $U_{\xi}^{0}$ and let $M$ be a $U_{\xi}^{\prime}$-module. Let $\lambda=\left(\lambda_{1}, \lambda_{2}, \ldots, \lambda_{n}\right) \in \mathbb{Z}^{n}, \sigma=\left(\sigma_{1}, \sigma_{2}, \ldots, \sigma_{n}\right) \in\{ \pm 1\}^{n}$. An element $m \in M$ is called to have weight $(\lambda, \sigma)$ if

$$
K_{i} m=\sigma_{i} \xi^{\lambda_{2} d_{2}} m, \quad\left[\begin{array}{c}
K_{i}, c \\
a
\end{array}\right] m=\sigma_{i}^{a}\left[\begin{array}{c}
\lambda_{i}+c \\
a
\end{array}\right]_{d_{l}, \xi} m
$$

for $i=1,2, \ldots, n, c \in \mathbb{Z}, a \in \mathbf{N}$. Denote by $M_{\lambda, \sigma}$ the set of all elements in $M$ of weight $(\lambda, \sigma)$. We call $(\lambda, \sigma)$ a weight of $M$ if $M_{\lambda, \sigma}$ is not zero. If an element $x$ in $U_{\xi}^{\prime}$ has degree $\gamma$, then obviously $x M_{\lambda, \sigma} \subseteq M_{\lambda-\gamma, \sigma}$.

As usual, for $(\lambda, \sigma),(\mu, \tau) \in \mathbf{Z}^{n} \times\{ \pm 1\}^{n}$, we write $(\lambda, \sigma) \leq(\mu, \tau)$ as well as $\lambda \leq \mu$ if $\mu-\lambda \in \mathbf{N} R^{+}$and $\sigma=\tau$. This defines a partial order in $\mathbb{Z}^{n} \times\{ \pm 1\}^{n}$ as well as in $\mathbb{Z}^{n}$.

2.4. Now we return to the algebra $\tilde{\mathbf{u}}_{\xi}$. Assume that $\mu=\left(\mu_{1}, \mu_{2}, \ldots, \mu_{n}\right) \in \mathbf{Z}^{n}$ and $\tau=\left(\tau_{1}, \tau_{2}, \ldots, \tau_{n}\right) \in\{ \pm 1\}^{n}$. Let $\tilde{I}_{\mu, \tau}^{+}$be the left ideal of $\tilde{\mathbf{u}}_{\xi}$ generated by 
the elements $E_{\alpha}, K_{i}-\tau_{i} \xi^{\mu_{i} d_{2}},\left[\begin{array}{c}K_{i}, c \\ a\end{array}\right]-\tau_{i}^{a}\left[\begin{array}{c}\mu_{i}+c \\ a\end{array}\right]_{d_{l}, \xi} \quad$ for $\alpha \in R_{l}^{+} \quad$ and $i=1,2, \ldots, n, c \in \mathbf{Z}, a \in \mathbf{N}$. Denote by $\tilde{Z}_{\xi}(\mu, \tau)$ the Verma module $\tilde{\mathbf{u}}_{\xi} / \tilde{I}_{\mu, \tau}^{+}$of $\tilde{\mathbf{u}}_{\xi}$ with highest weight $(\mu, \tau)$. We shall write $\tilde{1}_{\mu, \tau}$ for the image in $\tilde{Z}_{\xi}(\mu, \tau)$ of the neutral element $1 \in \tilde{\mathbf{u}}_{\xi}$. By 2.2 (iii), $\tilde{Z}_{\xi}(\mu, \tau)$ has dimension $\prod_{\alpha \in R^{+}} l_{\alpha}$. We denote by $\mathbf{Q}(\xi)_{1 \mu, \tau}$ the one dimensional $\tilde{\mathbf{u}}_{\xi}$-module on which all $E_{\alpha}, F_{\alpha}$ $\left(\alpha \in R_{l}^{+}\right)$act by scalar zero and $K_{i},\left[\begin{array}{c}K_{i}, c \\ a\end{array}\right](i=1,2, \ldots, n, c \in \mathbb{Z}, a \in \mathbf{N})$ act by scalar $\tau_{i}, \tau_{i}^{a}\left[\begin{array}{c}l_{i} \mu_{i}+c \\ a\end{array}\right]_{d_{i}, \xi}$, respectively.

Let $(\lambda, \sigma),(\mu, \tau) \in \mathbb{Z}^{n} \times\{ \pm 1\}^{n}$. Then (cf. [X1, Prop. 2.4, Prop. 2.9])

(i) The Verma module $\tilde{Z}_{\xi}(\lambda, \sigma)$ has a unique irreducible quotient module, denoted by $\tilde{L}_{\xi}(\lambda, \sigma)$ (this notation would cause no confusion with that in 2.2 (vii) since both are isomorphic when $\left.\lambda \in \mathbb{Z}_{+, 1}^{n}\right)$. Moreover $\tilde{L}_{\xi}(\mathbb{l} \mu, \tau) \simeq \mathbf{Q}(\xi)_{\mathbb{1} \mu, \tau}$.

(ii) We have

$$
\tilde{Z}_{\xi}(\lambda+\mathbf{l} \mu, \sigma \tau) \simeq \tilde{Z}_{\xi}(\lambda, \sigma) \otimes \tilde{L}_{\xi}(\mathbf{l} \mu, \tau) \simeq \tilde{Z}_{\xi}(\lambda, \tau) \otimes \tilde{L}_{\xi}(\mathbf{l} \mu, \sigma),
$$

where the meaning of $\sigma \tau \in\{ \pm 1\}^{n}$ is obvious.

(iii) We have

$$
\tilde{L}_{\xi}(\lambda+\mathbf{l} \mu, \sigma \tau) \simeq \tilde{L}_{\xi}(\lambda, \sigma) \otimes \tilde{L}_{\xi}(\mathbf{l} \mu, \tau) \simeq \tilde{L}_{\xi}(\lambda, \tau) \otimes \tilde{L}_{\xi}(\mathbb{l} \mu, \sigma) .
$$

(iv) Let $L$ be an irreducible $\tilde{\mathbf{u}}_{\xi}$-module such that $L$ is the direct sum of its weight spaces, then $L$ is isomorphic to certain $\tilde{L}_{\xi}(\lambda, \sigma)$. Two irreducible $\tilde{\mathbf{u}}_{\xi}$-modules $\tilde{L}_{\xi}(\lambda, \sigma)$ and $\tilde{L}_{\xi}(\mu, \tau)$ are isomorphic if and only if $(\lambda, \sigma)=(\mu, \tau)$.

(v) Remark. There is a natural bijection between the set of isomorphism classes of irreducible $\tilde{\mathbf{u}}_{\xi}$-modules and the set of isomorphism classes of irreducible $U_{\xi}^{0}$-modules (or equivalently, the set of maximal ideals of $U_{\xi}^{0}$ since $U_{\xi}^{0}$ is commutative). Note that the subalgebra $U_{\xi, l}^{\prime 0}$ of $U_{\xi}$ generated by $\left[\begin{array}{c}K_{i}, 0 \\ l_{i}\end{array}\right](i=1,2, \ldots, n)$ is isomorphic to a polynomial ring over $\mathbf{Q}(\xi)$ in $n$ variables, and $U_{\xi}^{0}$ is generated by all elements in $U_{\xi, l}^{\prime 0} \cup \mathbf{u}_{\xi}^{0}$.

Proof. Let $P$ be an irreducible $U_{\xi}^{0}$-module. We regard $P$ as a $\tilde{\mathbf{u}}_{\xi}^{b}=\mathbf{u}_{\xi}^{+} U_{\xi}^{0}$-module by defining $E_{\alpha} P=0$ for all $\alpha \in R_{l}^{+}$. Let $\tilde{Z}(P)=\tilde{\mathbf{u}}_{\xi} \otimes_{\tilde{\mathbf{u}}_{\xi}^{b}} P$. Then $\tilde{Z}(P)$ is a $\tilde{\mathbf{u}}_{\xi}$-module. Denote again by $P$ the image in $\tilde{Z}(P)$ of $P$. Then $\tilde{Z}(P)=\mathbf{u}_{\xi}^{-} P$. Let $M$ be a submodule of $\tilde{Z}(P)$. If $M \cap P \neq 0$, then $P \subseteq M$ since $P$ is an irreducible $U_{\xi}^{0}$-module. Thus $\tilde{Z}(P)=\mathbf{u}_{\xi}^{-} P \subseteq M$. Therefore, if $M$ is a proper submodule of $\tilde{Z}(P)$, then $M \cap P=0$. Thus $\tilde{Z}(P)$ has a unique maximal submodule, which is the sum of all proper submodules of $\tilde{Z}(P)$. Denote by $M(P)$ the maximal submodule of $\tilde{Z}(P)$ and denote by $\tilde{L}(P)$ the irreducible $\tilde{\mathbf{u}}_{\xi}$-module $\tilde{Z}(P) / M(P)$. 
Let $P, Q$ be irreducible $U_{\xi}^{0}$-modules. It is easy to see that $\tilde{L}(P) \simeq \tilde{L}(Q)$ if and only if $P \simeq Q$.

Now let $\tilde{L}$ be an irreducible $\tilde{\mathbf{u}}_{\xi}$-module. Let $P=\left\{x \in \tilde{L} \mid E_{\alpha} x=0\right.$ for all $\left.\alpha \in R_{l}^{+}\right\}$. Let $y$ be a nonzero element in $\tilde{L}$. Then $\mathbf{u}_{\xi}^{+} y$ is of finite dimension and we can find a nonzero element $x$ in $\mathbf{u}_{\xi}^{+} y$ such that $E_{\alpha} x=0$ for all $\alpha \in R_{l}^{+}$. Therefore $P$ is a nonzero space. Obviously $P$ is stable under $U_{\xi}^{0}$. Let $P^{\prime}$ be a proper submodule of $P$. Then $\tilde{\mathbf{u}}_{\xi} P^{\prime} \cap P=P^{\prime}$. So $\tilde{\mathbf{u}}_{\xi} P^{\prime}$ is a proper submodule of $\tilde{L}$. But $\tilde{L}$ is an irreducible $\tilde{\mathbf{u}}_{\xi}$-module, so $\tilde{\mathbf{u}}_{\xi} P^{\prime}=0$. In particular, $P^{\prime}=0$. Hence $P$ is an irreducible $U_{\xi}^{0}$-module. We have a natural $\tilde{\mathbf{u}}_{\xi}$-homomorphism $\tilde{Z}(P)=\tilde{\mathbf{u}}_{\xi} \otimes \tilde{\mathbf{u}}_{\xi}^{b} P \rightarrow \tilde{L}, u \otimes x \rightarrow u x$, which gives rise to an isomorphism between $\tilde{L}(P)$ and $\tilde{L}$.

The assertion is proved.

We need the following result to see that $\tilde{Z}_{\xi}(\lambda, \sigma)$ has a unique irreducible submodule.

Lemma 2.5. Given a nonzero element $y$ in $\mathbf{u}_{\xi}^{-}$we can find an element $x$ in $\mathbf{u}_{\xi}^{-}$such that $x y=F_{\kappa}$, where $F_{\kappa}=\prod_{\alpha \in R_{l}^{+}} F_{\alpha}^{\left(l_{\alpha}-1\right)}$, the product takes the order opposite to that in [L4, 4.3, pp.93-94].

Proof. Set $r:=\left|R^{+}\right|$. Let $\beta_{r-q+1}$ be the $q$-th root in the total order on $R^{+}$arranged in [loc. cit]. Then $\beta_{1}, \beta_{2}, \ldots, \beta_{r}$ give rise to a total order on $R^{+}$opposite to that in [loc. cit]. By 2.2 (iii),

$$
y=\sum_{\substack{0 \leq a_{m} \leq l_{\beta_{m}}-1 \\ 1 \leq m \leq r}} A\left(a_{1}, a_{2}, \ldots, a_{r}\right) F_{\beta_{1}}^{\left(a_{1}\right)} F_{\beta_{2}}^{\left(a_{2}\right)} \ldots F_{\beta_{r}}^{\left(a_{r}\right)}, \quad A\left(a_{1}, a_{2}, \ldots, a_{r}\right) \in \mathbf{Q}(\xi) .
$$

Let $\left(b_{1}, b_{2}, \ldots, b_{r}\right)$ be the minimal element in $\left\{\left(a_{1}, a_{2}, \ldots, a_{r}\right) \in \mathbf{Z}_{+}^{r} \mid A\left(a_{1}, a_{2}, \ldots, a_{r}\right)\right.$ $\neq 0\}$. (Here we use the lexicographical order in $\mathbf{Z}_{+}^{r}$ such that $(0,0, \ldots, 0,1)<$ $(0,0, \ldots, 1,0)<\cdots<(0,1, \ldots, 0,0)<(1,0, \ldots, 0,0)$.) Set $c_{1}=l_{\beta_{1}}-1-b_{1}, \ldots, c_{r}$ $=l_{\beta_{r}}-1-b_{r}$ and let $x^{\prime}=F_{\beta_{r}}^{\left(c_{r}\right)} \cdots F_{\beta_{2}}^{\left(c_{2}\right)} F_{\beta_{1}}^{\left(c_{1}\right)}$. Using commutation relations in [L4, 5.3-4, pp. 95-97] and [L4, Theorem 6.6 (iii), p. 104], we see $x^{\prime} y=$ $A\left(b_{1}, b_{2}, \ldots, b_{r}\right) x^{\prime} F_{\beta_{1}}^{\left(b_{1}\right)} F_{\beta_{2}}^{\left(b_{2}\right)} \cdots F_{\beta_{r}}^{\left(b_{r}\right)}=\theta F_{\kappa}$ for some nonzero number $\theta$ in $\mathbf{Q}(\xi)$. Then the element $x:=\theta^{-1} x^{\prime}$ satisfies our requirements.

Proposition 2.6. Let $(\lambda, \sigma) \in \mathbf{Z}^{n} \times\{ \pm 1\}^{n}$, then

(i) The Verma module $\tilde{Z}_{\xi}(\lambda, \sigma)$ has a unique irreducible submodule.

(ii) Assume that $\lambda$ is l-restricted. Then the unique irreducible submodule of $\tilde{Z}_{\xi}\left(2 \kappa+w_{0} \lambda, \sigma\right)$ is isomorphic to $\tilde{L}_{\xi}(\lambda, \sigma)$, where $w_{0}$ is the longest element of $W$.

Proof. (i) By Lemma 2.5, each submodule of $\tilde{Z}_{\xi}(\lambda, \sigma)$ contains the element $F_{\kappa} \tilde{1}_{\lambda, \sigma}$. So $\tilde{Z}_{\xi}(\lambda, \sigma)$ has a unique irreducible submodule which is generated by $F_{\kappa} \tilde{1}_{\lambda, \sigma}$.

(ii) Since $F_{\kappa}$ has degree $2 \kappa$, so $F_{\kappa} \tilde{1}_{2 \kappa+w_{0} \lambda, \sigma}$ has weight $\left(w_{0} \lambda, \sigma\right)$. 
According to the symmetries [L7, Prop. 5.2.7, p.45], the lowest weight of $L_{\xi}(\lambda, \sigma)$ is $\left(w_{0} \lambda, \sigma\right)$. According to 2.2 (vii), 2.4 (iii-iv) and the proof of (i) we see that the unique irreducible submodule of $\tilde{Z}_{\xi}\left(2 \kappa+w_{0} \lambda, \sigma\right)$ is isomorphic to $\tilde{L}_{\xi}(\lambda, \sigma)$.

\section{Corollary 2.7. Assume that $\lambda$ is 1-restricted. Then}

(i) There exists a nonzero element $y_{\lambda}$ in $\mathbf{u}_{\xi}^{-}$(unique up to a scalar) such that $y_{\lambda} \tilde{1}_{2 \kappa+w_{0} \lambda, \sigma}$ has weight $(\lambda, \sigma)$ and $E_{\alpha} y_{\lambda} \tilde{1}_{2 \kappa+w_{0} \lambda, \sigma}=0$ for all $\alpha \in R_{l}^{+}$. Necessarily $y_{\lambda} \tilde{1}_{2 \kappa+w_{0} \lambda, \sigma}$ generates the unique irreducible submodule of $\tilde{Z}_{\xi}\left(2 \kappa+w_{0} \lambda, \sigma\right)$.

(ii) There exists a nonzero element $y_{\lambda}^{\prime}$ in $\mathbf{⿴ 囗}_{\xi}^{-}$(unique up to a scalar) such that $y_{\lambda}^{\prime} \tilde{1}_{\kappa+\lambda, \sigma}$ has weight $\left(\kappa+w_{0} \lambda, \sigma\right)$ and $E_{\alpha} y_{\lambda}^{\prime} \tilde{1}_{\kappa+\lambda, \sigma}=0$ for all $\alpha \in R_{l}^{+}$. Necessarily $y_{\lambda}^{\prime} \tilde{1}_{\kappa+\lambda, \sigma}$ generates the unique irreducible submodule of $\tilde{Z}_{\xi}(\kappa+\lambda, \sigma)$.

We shall see that $y_{\lambda}^{\prime}=\eta x_{\lambda}^{\prime}$ for some non-zero number $\eta$ in $\mathbb{Q}(\xi)$ provided that every indecomposable component of the Cartan matrix $\left(a_{i j}\right)$ is not of type $G_{2}$ (Theorem 4.2 (ii), see 1.4 for the definition of $x_{\lambda}^{\prime}$ ).

Proposition 2.8. Let $\sigma \in\{ \pm 1\}^{n}$. Then

(i) The Verma module $\tilde{Z}_{\xi}(\kappa, \sigma)$ is an irreducible $\tilde{\mathbf{u}}_{\xi}$-module, i.e. $\tilde{Z}_{\xi}(\kappa, \sigma) \simeq$ $\tilde{L}_{\xi}(\kappa, \sigma)$.

(ii) As a $\tilde{\mathbf{u}}_{\xi}$-module, $V_{\xi}(\kappa, \sigma)$ is isomorphic to $\tilde{Z}_{\xi}(\kappa, \sigma)$. In particular, $V_{\xi}(\kappa, \sigma)$ is an irreducible $U_{\xi}$-module (cf. [L7, Prop. 35.4.4, p. 277].)

(iii) For every $\mu \in \mathbb{Z}_{+}^{n}$, the module $V_{\xi}(1 \mu+\kappa, \sigma)$ is an irreducible $U_{\xi}$-module.

Proof. (i) Note that $w_{0} \kappa=-\kappa$. By Prop. 2.6 (ii), the unique irreducible submodule of $\tilde{Z}_{\xi}(\kappa, \sigma)$ is isomorphic to $\tilde{L}_{\xi}(\kappa, \sigma)$. But $\tilde{Z}_{\xi}(\kappa, \sigma)_{\kappa, \sigma}$ is of one dimension, so the irreducible submodule of $\widetilde{Z}_{\xi}(\kappa, \sigma)$ is generated by $\tilde{1}_{\kappa, \sigma}$. Hence $\tilde{Z}_{\xi}(\kappa, \sigma)$ is irreducible and isomorphic to $\tilde{L}_{\xi}(\kappa, \sigma)$.

(ii) By the definitions of $\tilde{Z}_{\xi}(\kappa, \sigma)$ and of $V_{\xi}(\kappa, \sigma)$, we have a natural $\tilde{\mathbf{u}}_{\xi}$-module homomorphism $\tilde{Z}_{\xi}(\kappa, \sigma) \rightarrow V_{\xi}(\kappa, \sigma), \tilde{1}_{\kappa, \sigma} \rightarrow v_{\kappa, \sigma}$. The homomorphism is surjective according to 2.2 (ii) and to the definition of $V_{\xi}(\kappa, \sigma)$. Weyl's character formula tells us that the dimension of $V_{\xi}(\kappa, \sigma)$ is $\prod_{\alpha \in R^{+}} l_{\alpha}$. So the homomorphism is a $\tilde{\mathbf{u}}_{\xi}$-module isomorphism. This proves (ii).

(iii) By 1.2 (i), $L_{\xi}(\mathbb{1} \mu+\kappa, \sigma)$ is the unique irreducible quotient module of $V_{\xi}(\mathbb{1} \mu+\kappa, \sigma)$. Using (ii) and 2.2 (iv) we see that $L_{\xi}(\mathbb{1} \mu+\kappa, \sigma)$ is isomorphic to $L_{\xi}(\mathbb{l} \mu, \sigma) \otimes V_{\xi}(\kappa, \mathbb{1})$. Combining 2.2 (v), 1.2 (i) and 1.2 (iv), we know that the dimensions of $V_{\xi}(\mathbb{l} \mu+\kappa, \sigma)$ and $L_{\xi}(\mathbb{l} \mu, \sigma) \otimes V_{\xi}(\kappa, \mathbb{1})$ can be calculated by means of Weyl's character formula, they are equal. Hence $V_{\xi}(l \mu+\kappa, \sigma)$ is an irreducible $U_{\xi}$-module.

The proposition is proved.

The following result will not be used in the sequel of the paper. 
Theorem 2.9. (i) The algebra $\mathbf{u}_{\xi}$ is symmetric.

(ii) Let $\mathbf{k}$ be the two sided ideal of $\mathbf{u}_{\xi}$ generated by $K_{1}^{l_{1}}-1, K_{2}^{l_{2}}-1, \ldots, K_{n}^{l_{n}}-1$. Then the algebra $\mathbf{u}_{\xi}^{\prime}:=\mathbf{u}_{\xi} / \mathbf{k}$ is symmetric provided that all $K_{i}^{l_{2}}$ are central in $\mathbf{u}_{\xi}$.

Remark. The theorem was proved in [X1, Theorem 3.5] with some restrictions on $l$. Since [X1] is unpublished and Theorem 3.5 in [X1] was quoted in some papers, it might be good to represent here a version without restrictions on $l$. The proof is the same as in [X1].

Proof. (i) We need to construct a $\mathbf{Q}(\xi)$-bilinear form $\varphi$ on $\mathbf{u}_{\xi}$ such that

(a) $\varphi$ is associative, that is, $\varphi(x y, z)=\varphi(x, y z)$ for any $x, y, z \in \mathbf{u}_{\xi}$;

(b) $\varphi$ is no-degenerate, i.e. if $\varphi\left(x, x^{\prime}\right)=0$ (resp. $\varphi\left(x^{\prime}, x\right)=0$ ) for all $x^{\prime} \in \mathbf{u}_{\xi}$, then $x=0$;

(c) $\varphi$ is symmetric, that is, $\varphi(x, y)=\varphi(y, x)$.

Let $\beta_{1}, \beta_{2}, \ldots, \beta_{r}$ be as in the proof of Lemma 2.5. Set

$$
\begin{aligned}
\mathbf{Z}_{+, 1}^{r} & :=\left\{\left(a_{1}, a_{2}, \ldots, a_{r}\right) \in \mathbf{Z}^{r} \mid 0 \leq a_{1} \leq l_{\beta_{1}}-1, \ldots, 0 \leq a_{r} \leq l_{\beta_{r}}-1\right\}, \\
\mathbb{Z}_{+.21}^{n} & :=\left\{\left(h_{1}, h_{2}, \ldots, h_{n}\right) \in \mathbf{Z}^{n} \mid 0 \leq h_{1} \leq 2 l_{1}-1, \ldots, 0 \leq h_{n} \leq 2 l_{n}-1\right\} .
\end{aligned}
$$

For $A=\left(a_{1}, a_{2}, \ldots, a_{r}\right) \in \mathbf{Z}_{+, 1}^{r}$ and $H=\left(h_{1}, h_{2}, \ldots, h_{r}\right) \in \mathbf{Z}_{+, 21}^{n}$, we shall write $F_{A}, F_{A}^{\prime} ; E_{A}, E_{A}^{\prime} ; K_{H}$ for $F_{\beta_{1}}^{\left(a_{1}\right)} F_{\beta_{2}}^{\left(a_{2}\right)} \cdots F_{\beta_{r}}^{\left(a_{r}\right)}, F_{\beta_{r}}^{\left(a_{r}\right)} \cdots F_{\beta_{2}}^{\left(a_{2}\right)} F_{\beta_{1}}^{\left(a_{1}\right)} ; E_{\beta_{r}}^{\left(a_{r}\right)} E_{\beta_{r}-1}^{\left(a_{r}-1\right)} \cdots E_{\beta_{1}}^{\left(a_{1}\right)}$, $E_{\beta_{1}}^{\left(a_{1}\right)} \cdots E_{\beta_{r-1}}^{\left(a_{r-1}\right)} E_{\beta_{r}}^{\left(a_{r}\right)} ; K_{1}^{h_{1}} K_{2}^{h_{2}} \cdots K_{n}^{h_{n}}$, respectively. Let $\varphi_{0}$ be the $\mathbf{Q}(\xi)$-linear function of $\mathbf{u}_{\xi}$ defined by

$$
\varphi_{0}\left(F_{A} K_{H} E_{A^{\prime}}\right)= \begin{cases}1, & \text { if } F_{A} K_{H} E_{A^{\prime}}=F_{l} E_{l}, \\ 0, & \text { otherwise }\end{cases}
$$

where $l=\left(l_{\beta_{1}}-1, l_{\beta_{2}}-1, \ldots, l_{\beta_{r}}-1\right) \in \mathbf{Z}_{+, 1}^{r}$. Set $\varphi(x, y):=\varphi_{0}(x y)$. Obviously $\varphi$ is an associative $\mathbf{Q}(\xi)$-bilinear form on $\mathbf{u}_{\xi}$. We now show that $\varphi$ is nondegenerate on $\mathbf{u}_{\xi}$.

Let

$$
x=\sum_{\substack{A, A^{\prime} \in \mathbf{Z}_{+}^{r}, 1 \\ \boldsymbol{H} \in \mathbf{Z}_{+, 2 i}^{n}}} \theta\left(A, H, A^{\prime}\right) F_{A} K_{H} E_{A^{\prime}} \neq 0, \quad \theta\left(A, H, A^{\prime}\right) \in \mathbf{Q}(\xi)
$$

Let $B=\left(b_{1}, b_{2}, \ldots, b_{r}\right)$ be the minimal element in $\left\{A \in \mathbf{Z}_{+, 1}^{r} \mid \theta\left(A, H, A^{\prime}\right) \neq 0\right.$ for some $\left.H, A^{\prime}\right\}$ (for the lexicographical order on $\mathbf{Z}_{+}^{r}$ defined in the proof of Lemma 2.5), and let $B^{\prime}=\left(b_{1}^{\prime}, b_{2}^{\prime}, \ldots, b_{r}^{\prime}\right)$ be the minimal element in $\left\{A^{\prime} \in \mathbf{Z}_{+, 1}^{r} \mid\right.$ $\theta\left(B, H, A^{\prime}\right) \neq 0$ for some $\left.H\right\}$ (for the lexicographical order on $\mathbf{Z}_{+}^{r}$ opposite to that defined in the proof of Lemma 2.5). Set

$$
y_{1}=F_{\beta_{r}}^{\left(c_{r}\right)} \cdots F_{\beta_{2}}^{\left(c_{2}\right)} F_{\beta_{1}}^{\left(c_{1}\right)}, \quad y_{2}=E_{\beta_{1}}^{\left(c_{1}^{\prime}\right)} \cdots E_{\beta_{r-1}}^{\left(c_{r-1}^{\prime}\right)} E_{\beta_{r}}^{\left(c_{r}^{\prime}\right)},
$$

where $c_{1}=l_{\beta_{1}}-1-b_{1}, \ldots, c_{r}=l_{\beta_{r}}-1-b_{r}$, and $c_{1}^{\prime}=l_{\beta_{1}}-1-b_{1}^{\prime}, \ldots, c_{r}^{\prime}=$ $l_{\beta_{r}}-1-b_{r}^{\prime}$. By the proof of Lemma 2.5 we have 


$$
y_{2} y_{1} x=\theta_{1} y_{2} \sum_{\substack{A^{\prime} \in \mathbf{Z}^{r} \\ \boldsymbol{H} \in \mathbf{Z}_{+}^{++1}}} \theta\left(B, H, A^{\prime}\right) F_{l} K_{H} E_{A^{\prime}}
$$

for some nonzero number $\theta_{1} \in \mathbf{Q}(\xi)$.

Recall that in $U$ we have

$$
E_{i} F_{j}-F_{j} E_{i}=\delta_{i j} \frac{K_{i}-K_{i}^{-1}}{v^{d_{i}}-v^{-d_{i}}} .
$$

This implies the following

(*) Let $\alpha, \beta \in R^{+}$. Then in $U$ we have

$$
E_{\alpha} F_{\beta}=F_{\beta} E_{\alpha}+\sum \sigma(F, K, E) F K E, \quad \sigma(F, K, E) \in \mathbf{Q}\left[v, v^{-1}\right],
$$

where in the sum $K$ runs through a finite subset of $U^{0} \cap U_{\mathbb{Q}\left[v, v^{-1}\right]}, F$ (resp. $E$ ) runs through a finite subset of homogenous elemets of $U^{-} \cap U_{\mathbb{Q}\left[v, v^{-1}\right]}$ (resp. $\left.U^{+} \cap U_{\mathbf{Q}\left[v, v^{-1}\right]}\right)$, and $\sigma(F, K, E)=0$ if $\operatorname{deg}(F) \geq \beta$ or $\operatorname{deg}(E) \leq-\alpha$.

From (*) and 2.2 (iii) we get

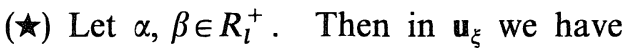

$$
E_{\alpha} F_{\beta}=F_{\beta} E_{\alpha}+\sum_{\substack{A, A^{\prime} \in \mathbb{Z}_{+, 1}^{r} \\ \boldsymbol{H} \in \mathbf{Z}_{+, 2 i}^{n}}} \sigma\left(A, H, A^{\prime}\right) F_{A} K_{H} E_{A^{\prime}}, \quad \sigma\left(A, H, A^{\prime}\right) \in \mathbb{Q}(\xi),
$$

where $\sigma\left(A, H, A^{\prime}\right)=0$ if $\operatorname{deg}\left(F_{A}\right) \geq \beta$ or $\operatorname{deg}\left(E_{A^{\prime}}\right) \leq-\alpha$.

Repeatedly using ( $\star$ ) we get

(†) Let $B, C \in \mathbf{Z}_{+, 1}^{r}$ and let $y=E_{B}$ or $E_{B}^{\prime}, z=F_{C}$ or $F_{C}^{\prime}$. Then in $\mathbf{u}_{\xi}$ we have

$$
y z=z y+\sum_{\substack{A, A^{\prime} \in \mathbb{Z}^{r}, 1 \\ H \in \mathbf{Z}_{+}^{n}, 21}} \sigma\left(A, H, A^{\prime}\right) F_{A} K_{H} E_{A^{\prime}}, \quad \sigma\left(A, H, A^{\prime}\right) \in \mathbb{Q}(\xi),
$$

where $\sigma\left(A, H, A^{\prime}\right)=0$ if $\operatorname{deg}\left(F_{A}\right) \geq \operatorname{deg}(z)$ or $\operatorname{deg}\left(E_{A^{\prime}}\right) \leq-\operatorname{deg}(y)$.

By $(\dagger)$ we get

$$
y_{2} F_{l}=F_{l} y_{2}+\sum_{\substack{A, A^{\prime} \in \mathbb{Z}_{+}^{r} \\ A \neq l \\ H \in \mathbb{Z}_{+}^{n}, 21}} \eta\left(A, H, A^{\prime}\right) F_{A} K_{H} E_{A^{\prime}}, \quad \eta\left(A, H, A^{\prime}\right) \in \mathbb{Q}(\xi) .
$$

As in the proof of Lemma 2.5 we see $\theta\left(B, H, A^{\prime}\right) y_{2} E_{A^{\prime}} \neq 0$ implies that $A^{\prime}=B^{\prime}$ and $y_{2} E_{B^{\prime}}=\theta_{2} E_{l}$ for some nonzero number $\theta_{2} \in \mathbf{Q}(\xi)$. Thus

$$
y_{2} y_{1} x=\theta_{1} \theta_{2} \sum_{H \in \mathbb{Z}_{+}^{n}, 21} \theta\left(B, H, B^{\prime}\right) F_{l} K_{H} E_{l}+\sum_{\substack{A, A^{\prime} \in \mathbb{Z}_{+}^{r}, 1 \\ A \neq \neq \\ H \in \mathbf{Z}_{+, 21}^{n}}} \eta^{\prime}\left(A, H, A^{\prime}\right) F_{A} K_{H} E_{A^{\prime}},
$$

where $\eta^{\prime}\left(A, H, A^{\prime}\right) \in \mathbb{Q}(\xi)$. Let $I \in \mathbb{Z}_{+, 21}^{n}$ be such that $\theta\left(B, I, B^{\prime}\right) \neq 0$. By the definition of $\varphi$ we see $\varphi\left(K_{I}^{-1} y_{2} y_{1}, x\right) \neq 0$. We also have $\varphi\left(x, K_{I}^{-1} y_{2} y_{1}\right) \neq 0$ since $\varphi$ is symmetric by the following argument. 
Note that the elements $E_{A}^{\prime} K_{H} F_{A^{\prime}}^{\prime}\left(A, A^{\prime} \in \mathbf{Z}_{+, 1}^{r}, H \in \mathbf{Z}_{+, 21}^{n}\right)$ also form a $\mathbf{Q}(\xi)$-basis of $\mathbf{u}_{\xi}$. Let $A=\left(a_{k}\right), B=\left(b_{k}\right), P=\left(p_{k}\right), Q=\left(q_{k}\right)$ be elements in $\mathbf{Z}_{+, \mathbf{I}}^{r}$ and let $H=\left(h_{i}\right), H^{\prime}=\left(h_{i}^{\prime}\right)$ be elements in $\mathbf{Z}_{+, 21}^{n}$. Using commutation relations in [L4, 5.3-5.4, pp.95-97], Theorem 6.6 in [L4, pp. 103-104] and using ( $\dagger$ ), we see that $\varphi\left(F_{A} K_{H} E_{P}, E_{Q}^{\prime} K_{H^{\prime}} F_{B}^{\prime}\right)=\varphi\left(E_{Q}^{\prime} K_{H^{\prime}} F_{B}^{\prime}, F_{A} K_{H} E_{P}\right)=0$ if one of the following three cases happens: (a) $K_{H} K_{H^{\prime}} \neq 1$, (b) $\sum_{k=1}^{r}\left(a_{k}+b_{k}\right) \beta_{k} \neq 2 \kappa$, (c) $\sum_{k=1}^{r}\left(p_{k}+q_{k}\right) \beta_{k} \neq 2 \kappa$. Using [L4, Theorem 6.6, pp. 103-104] and commutation relations in $[\mathrm{L} 4,5.3-5.4$, pp.95-97] and induction on $Q$ (resp. $B$ ) we know that (d) $E_{P} E_{Q}^{\prime}=E_{Q}^{\prime} E_{P}$ (resp. $\left.F_{A} F_{B}^{\prime}=F_{B}^{\prime} F_{A}\right)$ if $\sum_{k=1}^{r}\left(p_{k}+q_{k}\right) \beta_{k}=2 \kappa$ (resp. $\left.\sum_{k=1}^{r}\left(a_{k}+b_{k}\right) \beta_{k}=2 \kappa\right)$. By this and $(\dagger)$, and noting that the coefficients of $E_{P} E_{Q}^{\prime}, F_{B}^{\prime} F_{A}$ in $K_{H} E_{P} E_{Q}^{\prime} K_{H}^{-1}, K_{H}^{-1} F_{B}^{\prime} F_{A} K_{H}$ are the same when $\sum_{k=1}^{r}\left(a_{k}+\right.$ $\left.b_{k}\right) \beta_{k}=\sum_{k=1}^{r}\left(p_{k}+q_{k}\right) \beta_{k}=2 \kappa$, we see that $\varphi\left(F_{A} K_{H} E_{P}, E_{Q}^{\prime} K_{H^{\prime}} F_{B}^{\prime}\right)=\varphi\left(E_{Q}^{\prime} K_{H^{\prime}} F_{B}^{\prime}\right.$, $\left.F_{A} K_{H} E_{P}\right)$ if $\sum_{k=1}^{r}\left(a_{k}+b_{k}\right) \beta_{k}=\sum_{k=1}^{r}\left(p_{k}+q_{k}\right) \beta_{k}=2 \kappa$ and $K_{H} K_{H^{\prime}}=1$. Therefore $\varphi$ is symmetric. Part (i) is proved.

(ii) Since all $K_{i}^{l_{2}}$ are central in $\mathbf{u}_{\xi}$, the images in $\mathbf{u}_{\xi}^{\prime}$ of the elements $F_{A} K_{H} E_{A^{\prime}}\left(A, A^{\prime} \in \mathbf{Z}_{+, 1}^{r}, H \in \mathbf{Z}_{+, 1}^{n}\right)$ form a $\mathbf{Q}(\xi)$-basis of $\mathbf{u}_{\xi}^{\prime}$, the proof of (i) is also valid to $\mathbf{u}_{\xi}^{\prime}$.

The theorem is proved.

\section{§3. Category of Finite Dimensional $U_{\xi}$-modules of Type 1}

3.1. Let $M$ be a finite dimensional $U_{\xi}$-module. For $\lambda=\left(\lambda_{1}, \ldots, \lambda_{n}\right) \in \mathbf{Z}^{n}$, $\sigma=\left(\sigma_{1}, \sigma_{2}, \ldots, \sigma_{n}\right)$ in $\{ \pm 1\}^{n}$, let $M_{(\lambda, \sigma)}$ be the set of all elements $m$ in $M$ satisfying

$$
K_{i} m=\sigma_{i} \xi^{d_{1} \lambda_{2}} m,\left(\left[\begin{array}{c}
K_{i}, c \\
a
\end{array}\right]-\sigma_{i}^{a}\left[\begin{array}{c}
\lambda_{i}+c \\
a
\end{array}\right]_{d_{l}, \xi}\right)^{k} m=0,
$$

for $i=1,2, \ldots, n, c \in \mathbb{Z}$ and some $k \in \mathbf{N}$. Then we have

(i) $M=\bigoplus_{\substack{\lambda \in \mathbf{Z}^{n} \\ \sigma \in\{ \pm 1\}^{n}}} M_{(\lambda, \sigma)}$, and $E_{i}^{(a)} M_{(\lambda, \sigma)} \subseteq M_{\left(\lambda+a \alpha_{1}, \sigma\right)}, \quad F_{i}^{(a)} M_{(\lambda, \sigma)} \subseteq M_{\left(\lambda-a \alpha_{1}, \sigma\right)}$. Therefore, for a fixed $\sigma \in\{ \pm 1\}^{n}$, the space $M^{\sigma}=\oplus_{\lambda \in \mathbf{Z}^{n}} M_{(\lambda, \sigma)}$ is a submodule of $M$ and $M=\oplus_{\sigma \in\{ \pm 1\}^{n}} M^{\sigma}$. [L2, Prop. 5.1 and its proof, pp.65-67].

(ii) Obviously, $M_{\lambda, \sigma} \neq 0$ if and only if $M_{(\lambda, \sigma)} \neq 0$. So the set $P(M):=\left\{\lambda \in \mathbf{Z}^{n} \mid\right.$ $M_{(\lambda, \sigma)} \neq 0$ for some $\left.\sigma \in\{ \pm 1\}^{n}\right\}$ is stable under the action of $W$ [L7, Prop. 5.2.7, p. 45].

In $U_{\xi}$ we have $K_{i}^{2 l_{2}}=1$. For each $\sigma=\left(\sigma_{1}, \sigma_{2}, \ldots, \sigma_{n}\right)$ in $\{ \pm 1\}^{n}$, set $\tilde{M}^{\sigma}:=\left\{m \in M \mid K_{i}^{l^{2}} m=\sigma_{i} m\right.$ for $\left.i=1,2, \ldots, n\right\}$. When all $K_{i}^{l_{1}}$ are central in $U_{\xi}$, $\tilde{M}^{\sigma}$ is a submodule of $M$ and $M=\bigoplus_{\sigma \in\{ \pm 1\}^{n}} \tilde{M}^{\sigma}$. If $l$ is odd (prime to 3 if there is type $G_{2}$ involved), all $l_{i}$ are equal to $l$ and all $K_{i}^{l}$ are central in $U_{\xi}$, in this case we have $M^{\sigma}=\tilde{M}^{\sigma}$. When some $K_{i}^{l_{2}}$ are not central in $U_{\xi}$, in general $\tilde{M}^{\sigma}$ is not a $U_{\xi}$-submodule of $M$, since we can find $j$ in $[1, n]$ such 
that $K_{i}^{l_{2}} E_{j}=\xi^{d_{2} a_{1} l_{l}} E_{j} K_{i}^{l_{2}}=-E_{j} K_{i}^{l_{2}}$ and $K_{i}^{l_{2}} F_{j}=\xi^{-d_{i} a_{1 \jmath} l_{2}} F_{j} K_{i}^{l_{2}}=-F_{j} K_{i}^{l_{2}}$.

We say that $M$ has type $\sigma$ if $M=M^{\sigma}$. All finite dimensional $U_{\xi}$-modules of type $\sigma$ with usual $U_{\xi}$-module homomorphisms form a category of $U_{\xi}$-modules, denoted by $\mathscr{C}_{\sigma}$. Clearly, the map $M \rightarrow M \otimes \mathbf{Q}(\xi)_{\sigma}$ gives rise to an isomorphism between the categories $\mathscr{C}_{1}$ and $\mathscr{C}_{\sigma}$ [APW, 1.6, pp.6-7]. What is more, the $\mathbf{Q}(\xi)$-algebra automorphism $U_{\xi} \rightarrow U_{\xi}$ defined by $E_{i}^{(a)} \rightarrow \sigma_{i}^{a} E_{i}^{(a)}$, $F_{i}^{(a)} \rightarrow F_{i}^{(a)}, K_{i} \rightarrow \sigma_{i} K_{i}(i=1,2, \ldots, n, a \geq 0)$ interchanges the $U_{\xi}$-modules of type 1 to those of type $\sigma[\mathrm{L} 2,4.6, \mathrm{p} .65]$.

Therefore, it suffices to work with the category $\mathscr{C}_{1}$ of $U_{\xi}$-modules. Note that $V_{\xi}(\lambda, \mathbb{1}), L_{\xi}(\lambda, \mathbb{1}) \in$ ob $\mathscr{C}_{1}$ for each $\lambda \in \mathbb{Z}_{+}^{n}$. We shall drop the index $\mathbb{1}$ in all notations involving it. So $\mathscr{C}, V_{\xi}(\lambda), L_{\xi}(\lambda), v_{\lambda}$, etc. will stand for $\mathscr{C}_{\mathbb{1}}, V_{\xi}(\lambda, \mathbb{1})$, $L_{\xi}(\lambda, 1), v_{\lambda, 1}$, etc. respectively. One main result of the section is the following, which will be proved after establishing Lemma 3.4.

Theorem 3.2. Let $\mu \in \mathbb{Z}_{+}^{n}$.

(i) The module $V_{\xi}(1 \mu+\kappa)$ is injective as well as projective in the category $\mathscr{C}$.

(ii) The category $\mathscr{C}$ has enough injective objects and enough projective objects as well.

(iii) In $\mathscr{C}$ each injective object is also a projective object and vice versa.

(iv) Every module $M$ in ob $\mathscr{C}$ is integrable (i.e. $M=\bigoplus_{\lambda \in \mathbf{Z}^{n}} M_{\lambda}$ and $E_{i}^{(a)}, F_{i}^{(a)}$ are locally nilpotent on $M$ for $i=1,2, \ldots, n, a \geq 1$ ).

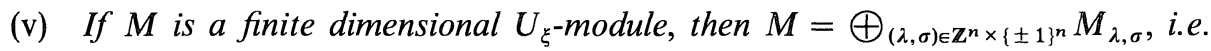
$M$ is integrable.

(vi) Let $E$ be an injective object in $\mathscr{C}$, then $E$ has a submodule filtration $0=E_{k} \subset E_{k-1} \subset \cdots \subset E_{2} \subset E_{1}=E$ such that $E_{a} / E_{a+1} \simeq V_{\xi}\left(v_{a}\right)$ for some $v_{a} \in \mathbb{Z}_{+}^{n}$, $a=1, \ldots, k-1$.

Remark. When $l$ is a power of a prime number, the theorem is proved in $[\mathrm{APW}, 9.8$, p. $44 ; 9.12$, p. 45$]$.

3.3. Let $M$ be a $U_{\xi}$-module of type 1. A nonzero element $m$ in $M$ is called primitive if $m \in M_{\lambda}$ for some $\lambda \in \mathbb{Z}^{n}$ and $E_{i}^{(a)} m=0$ for $i=1,2, \ldots, n, a \geq 1$. We have

(i) Let $M$ be an integrable or finite dimensional $U_{\xi}$-module of type 1. Assume that $m$ is a primitive element of weight $\lambda$. Then $\lambda \in \mathbb{Z}_{+}^{n}$ and there is a unique $U_{\xi}$-module homomorphism $V_{\xi}(\lambda) \rightarrow M$ which carries $v_{\lambda}$ to $m$. [L7, Prop. 3.5.8, p. 33].

Given a finite dimensional $U_{\xi}$-module $E$ of type $\mathbb{1}$, we define the dual modules $E^{*}, E^{\star}$ as in [APW, 1.18, p.9] by means of the antipode $S$ of $U_{\xi}$ and its inverse $S^{-1}$ respectively. Then [APW, 1.18, p.9-10]

(ii) We have $\left(E^{*}\right)^{\star} \simeq E \simeq\left(E^{\star}\right)^{*}$.

(iii) For any $U_{\xi}$-modules $M, N$, one has 


$$
\begin{aligned}
& \operatorname{Hom}_{U_{\xi}}(M, N \otimes E) \simeq \operatorname{Hom}_{U_{\xi}}\left(M \otimes E^{\star}, N\right), \\
& \operatorname{Hom}_{U_{\xi}}\left(E^{*} \otimes M, N\right) \simeq \operatorname{Hom}_{U_{\xi}}(M, E \otimes N) .
\end{aligned}
$$

The following assertion is well known.

(iv) For $\lambda \in \mathbb{Z}_{+}^{n}$ we have

$$
L_{\xi}(\lambda)^{*} \simeq L_{\xi}(\lambda)^{\star} \simeq L_{\xi}\left(-w_{0} \lambda\right)
$$

For later uses we prove that some $U_{\xi}$-modules admit filtrations of Weyl modules.

For $U$-modules we define weight spaces, primitive elements, and their types as usual. Here we only consider $U$-modules of type 1. For a $U$-module $M$ of type 1 we denote $M_{\lambda}$ the $\lambda$-weight space of $M$.

Let $\mathscr{A}=\mathbb{Z}\left[v, v^{-1}\right]$ and let $U_{\mathscr{A}}$ be the $\mathscr{A}$-subalgebra of $U$ generated by the elements $E_{i}^{(a)}, F_{i}^{(a)}, K_{i}, K_{i}^{-1}$ for $i=1, \ldots, n, a \in \mathbf{N}$. For $\lambda \in \mathbf{Z}_{+}^{n}$, let $V(\lambda)$ be an irreducible $U$-module of type $\mathbb{1}$ with highest weight $\lambda$, and let $v_{\lambda} \in V(\lambda)$ be a nonzero element of weight $\lambda$. Define $V(\lambda)_{\mathscr{A}}=U_{\mathscr{A}} v_{\lambda}$. Let $B(\lambda)$ be the canonical basis of $V(\lambda)$ containing $v_{\lambda}$, then $B(\lambda)$ is an $\mathscr{A}$-basis of $V(\lambda)_{\mathscr{A}}$ (see [K, Section 7.2 and Lemma 7.3.1; L5, Theorem 8.10; L7, 19.3.1, p. 170]).

We have

(v) Let $(M, B)$ be a based module of $U$ (see [L7, 27.1.2, p. 214] for definition) and let $M_{\mathscr{A}}$ be the $\mathscr{A}$-submodule of $M$ generated by $B$. (By definition $M_{\mathscr{A}}$ is stable under $U_{\mathscr{A}}$.) Then $M_{\mathscr{A}}$ has a filtration of $U_{\mathscr{A}}$-submodules

$$
0=M_{\mathscr{A}, 0} \subset M_{\mathscr{A}, 1} \subset M_{\mathscr{A}, 2} \subset \cdots \subset M_{\mathscr{A}, h}=M_{\mathscr{A}}
$$

such that all $M_{\mathscr{A}, 1}, \ldots, M_{\mathscr{A}, h}$ are free $\mathscr{A}$-modules and $M_{\mathscr{A}, a} / M_{\mathscr{A}, a-1} \simeq V\left(\delta_{a}\right)_{\mathscr{A}}$ for some $\delta_{a} \in \mathbb{Z}_{+}^{n}, a=1, \ldots, h$.

Proof. Let $\lambda \in \mathbb{Z}_{+}^{n}$ be such that $M_{\lambda} \neq 0$ and such that $\lambda$ is maximal with this property. Let $b \in B \cap M_{\lambda}$ and let $M_{1}=U b$ be the submodule of $M$ generated by $b$. We have $E_{i} b=0$ for all $i$ in $[1, n]$ by the maximality of $\lambda$. Hence there is a unique $U$-homomorphism $\phi: V(\lambda) \rightarrow M$ which carries $v_{\lambda}$ to $b$ [L7, Prop. 3.5.8, p. 33].

By [L7, Prop. 27.1.7, p. 215], $B_{1}=B \cap M_{1}$ is a basis of $M_{1}$ and $\phi$ defines an isomorphism $V(\lambda) \simeq M_{1}$ which carries $B(\lambda)$ to $B_{1}$. Since $B(\lambda)$ is an $\mathscr{A}$-basis of $V(\lambda)_{\mathscr{A}}$, we see $B_{1}$ is an $\mathscr{A}$-basis of $U_{\mathscr{A}} b$. Let $M_{\mathscr{A}, 1}=U_{\mathscr{A}} b$ and let $M^{\prime}=M / M_{1}$. Denote by $B^{\prime}$ the image in $M^{\prime}$ of $B-B_{1}$. Then $\left(M^{\prime}, B^{\prime}\right)$ is a based module [L7, 27.1.4, p. 215]. Since $M_{\mathscr{A}} / M_{\mathscr{A}, 1} \simeq M_{\mathscr{A}}^{\prime}$, using induction on $\operatorname{dim} M$ we see the required filtration exists.

The assertion is proved.

For $a=1,2, \ldots, h$, let $M_{\xi, a}=M_{\mathscr{A}, a} \otimes_{\mathscr{A}} \mathbf{Q}(\xi)$, and set $M_{\xi}=M_{\mathscr{A}} \otimes_{\mathscr{A}} \mathbf{Q}(\xi)$. Then $M_{\xi, a}$ is a $U_{\xi}$-module. Noting that $V_{\xi}(\lambda) \simeq V(\lambda)_{\mathscr{A}} \otimes_{\mathscr{A}} \mathrm{Q}(\xi)$, by (v) we see 
(vi) The $U_{\xi}$-module $M_{\xi}$ has a filtration of submodules

$$
0=M_{\xi, 0} \subset M_{\xi, 1} \subset M_{\xi, 2} \subset \cdots \subset M_{\xi, h}=M_{\xi}
$$

such that $M_{\xi, a} / M_{\xi, a-1} \simeq V_{\xi}\left(\delta_{a}\right)$ for some $\delta_{a} \in \mathbf{Z}_{+}^{n}, a=1, \ldots, h$.

Let $\lambda, \mu \in \mathbb{Z}_{+}^{n}$ and let $M=V(\lambda) \otimes V(\mu)$. According to [L7, 27.3.3, p. 221], there exists a basis $B$ of $M$ such that $(M, B)$ is a based module and such that the $\mathscr{A}$-submodule of $M$ generated by $B$ is equal to $V(\lambda)_{\mathscr{A}} \otimes_{\mathscr{A}} V(\mu)_{\mathscr{A}}$. (Note that $B(\lambda)$ and $B(\mu)$ are $\mathscr{A}$-bases of $V(\lambda)_{\mathscr{A}}$ and $V(\mu)_{\mathscr{A}}$ respectively.) Since $V_{\xi}(\lambda) \otimes V_{\xi}(\mu) \simeq\left(V(\lambda)_{\mathscr{A}} \otimes_{\mathscr{A}} V(\mu)_{\mathscr{A}}\right) \otimes_{\mathscr{A}} \mathbf{Q}(\xi)$, by (vi) we get

(vii) Let $\lambda, \mu \in \mathbb{Z}_{+}^{n}$. Then the $U_{\xi}$-module $V:=V_{\xi}(\lambda) \otimes V_{\xi}(\mu)$ has a filtration of submodules

$$
0=V_{0} \subset V_{1} \subset V_{2} \subset \cdots \subset V_{h}=V
$$

such that $V_{a} / V_{a-1} \simeq V_{\xi}\left(\delta_{a}\right)$ for some $\delta_{a} \in \mathbb{Z}_{+}^{n}, a=1, \ldots, h$.

Lemma 3.4. Let $M$ be a finite dimensional $U_{\xi}$-module of type $\mathbb{1}$ and let $\mu \in \mathbb{Z}_{+}^{n}$.

(i) Assume that $V_{\xi}(1 \mu+\kappa)$ is a submodule of $M$. Then $V_{\xi}(1 \mu+\kappa)$ is a direct summand of $M$, i.e. there exists a submodule $M^{\prime}$ of $M$ such that $M$ is isomorphic to $V_{\xi}(1 \mu+\kappa) \oplus M^{\prime}$.

(ii) Assume that $V_{\xi}(1 \mu+\kappa)$ is a quotient module of $M$. Then $\left.V_{\xi}(1)+\kappa\right)$ is a direct summand of $M$.

(iii) Assume that $V_{\xi}(1 \mu+\kappa)$ is a composition factor of $M$. Then $V_{\xi}(1 \mu+\kappa)$ is a direct summand of $M$.

Proof. Let $v=-w_{0}(\mu)$. By 3.2 (iv) we have $V_{\xi}(1 \mu+\kappa)^{*} \simeq V_{\xi}(\mathbb{l} \mu+\kappa)^{\star} \simeq$ $V_{\xi}(\mathbf{l} v+\kappa)$ since $V_{\xi}(\mathbf{l} \mu+\kappa)$ is irreducible (Prop. 2.8 (iii)) and $w_{0}(\mathbf{l} \mu+\kappa)=$ $-\mathbf{l} v-\kappa$. Thus part (i) and part (ii) are equivalent by 3.3 (ii). We give a proof of part (i).

(i) By induction on $\operatorname{dim}_{\mathbf{Q}(\xi)} M$ we may assume that $M / V_{\xi}(1 \mu+\kappa)$ is irreducible. One of the following three cases must happen.

(a) There is a maximal weight $\lambda$ in $P(M)$ such that $\lambda \neq 1 \mu+\kappa$.

(b) $\mathbb{l} \mu+\kappa$ is the unique maximal weight in $P(M)$ and $\operatorname{dim}_{\mathbf{Q}(\xi)} M_{(\mathbf{l} \mu+\kappa)}=1$.

(c) $\mathbf{l} \mu+\kappa$ is the unique maximal weight in $P(M)$ and $\operatorname{dim}_{\mathbf{Q}(\xi)} M_{(\mathbf{l} \mu+\kappa)}=2$.

Case (a). By 3.1 (ii), $M_{\lambda} \neq 0$. Choose a nonzero element $m$ in $M_{\lambda}$, then $m$ is a primitive element. Let $M^{\prime}$ be the submodule of $M$ generated by $m$. We claim that $M^{\prime} \cap V_{\xi}(\mathbf{l} \mu+\kappa)=\{0\}$. Otherwise, $M^{\prime} \cap V_{\xi}(1 \mu+\kappa)=V_{\xi}(\mathbb{l} \mu+\kappa)$. Then we can find an element $y$ in $U_{\xi}^{-}$such that $v_{\mathbf{l} \mu+\kappa}=y m$. Note that $F_{\kappa} v_{\mathbf{l} \mu+\kappa} \neq 0$. Using 2.2 (ii-iii) we see that $F_{\kappa} v_{\mathbf{1} \mu+\kappa}=F_{\kappa} y^{\prime} m$ for some element $y^{\prime}$ in $U_{\xi, l}^{-}$. Therefore $\lambda=\mathbf{l} \tau+\mathbb{l} \mu+\kappa$ for certain nonzero element $\tau$ in $\mathbb{Z}^{n}$. By Prop. 2.8 (iii) and 3.3 (i), $M^{\prime}$ is irreducible. A contradiction to the assumption $M^{\prime} \cap V_{\xi}(\mathbf{l} \mu+\kappa)=V_{\xi}(\mathbf{l} \mu+\kappa)$. Hence $M^{\prime} \cap V_{\xi}(\mathbb{I} \mu+\kappa)=\{0\}$. In addition we 
have $M \simeq V_{\xi}(\mathbf{l} \mu+\kappa) \oplus M^{\prime}$ and $M^{\prime}$ is irreducible.

Case (b). By 3.1 (ii), all the four spaces $M_{\mathbf{I} \mu+\kappa}, M_{(\mathbf{1} \mu+\kappa)}, M_{-1 v-\kappa}, M_{(-1 v-\kappa)}$ are of one dimension. So $M_{1 v+\kappa}^{*}$ is equal to $M_{(1 v+\kappa)}^{*}$ and is of one dimension. By 3.1 (ii), $\mathbf{l} v+\kappa$ is the unique maximal weight in $P\left(M^{*}\right)=$ $-P(M)$. Let $M_{1}$ be an irreducible submodule of $M^{*}$ such that $M^{*} / M_{1}$ is isomorphic to $V_{\xi}(1 \mu+\kappa)^{*}\left(\simeq V_{\xi}(1 v+\kappa)\right)$. By our assumptions on $M$ we have $\mathbf{l} v+\kappa \notin P\left(M_{1}\right)$. Choose a nonzero element $m$ in $M_{1 v+\kappa}^{*}$, then $m$ is a primitve element in $M^{*}$ and generates a submodule $M_{2}$ of $M^{*}$. By Prop. 2.8 (iii) and 3.3 (i), $M_{2}$ is isomorphic to $V_{\xi}(\mathrm{l} v+\kappa)\left(\simeq V_{\xi}(\mathbf{l} \mu+\kappa)^{*}\right)$. Hence $M^{*}$ is isomorphic to $V_{\xi}(\mathbf{l} v+\kappa) \oplus M_{1}$. Note that $V_{\xi}(\mathbf{l} v+\kappa)^{\star} \simeq V_{\xi}(\mathbf{l} \mu+\kappa)$, by 3.3 (ii) we see that $M$ is isomorphic to $V_{\xi}(l \mu+\kappa) \oplus M_{1}^{\star}$.

Case (c). Set $\mu_{i}:=\left\langle\mu, \alpha_{i}^{\vee}\right\rangle$ for $i=1,2, \ldots, n$. By 3.1 (ii) we have $F_{i}^{\left(l_{l} \mu_{l}+l_{2}\right)} M_{(\mathbf{l} \mu+\kappa)}=0$ for all $i$. Using 3.1 (i) and our assumption on $\mathbf{l} \mu+\kappa$ we see

$$
\left[\begin{array}{c}
K_{i}, 0 \\
\mu_{i} l_{i}+l_{i}
\end{array}\right] M_{(\mathbf{l} \mu+k)}=E_{i}^{\left(l_{\imath} \mu_{i}+l_{\imath}\right)} F_{i}^{\left(l_{\imath} \mu_{i}+l_{\imath}\right)} M_{(\mathbf{l} \mu+\kappa)}=0 .
$$

But in $U_{\xi}$ we have [L2, 4.2 (f), p.63; L7, Lemma 34.1 .2 (b), p. 265]

$$
\left[\begin{array}{c}
K_{i}, 0 \\
\mu_{i} l_{i}+l_{i}
\end{array}\right]=\frac{\xi^{-\frac{1}{2} d_{l} l_{1}^{2} \mu_{i}\left(\mu_{i}+1\right)}}{\left(\mu_{i}+1\right) !} \prod_{j=0}^{\mu_{2}}\left[\begin{array}{c}
K_{i},-j l_{i} \\
l_{i}
\end{array}\right] .
$$

By [L2, 4.2 (d), 4.2 (c), p. 63, L7, Lemma 34.1 .2 (b), p. 265] we see (*) On $M_{(1 \mu+\kappa)}$ the following equality holds

$$
\left[\begin{array}{c}
K_{i},-j l_{i} \\
l_{i}
\end{array}\right]=\xi^{-j l_{l}^{2} d_{\imath}}\left(\left[\begin{array}{c}
K_{i}, 0 \\
l_{i}
\end{array}\right]-\xi^{d_{1} l_{l}^{2}\left(\mu_{i}+1\right)+d_{l} l_{i}\left(l_{2}-1\right)} j\right) .
$$

$\left.\begin{array}{l}\text { By definition, } \\ {\left[K_{i},-j l_{i}\right]} \\ l_{i}, 0\end{array}\right]-\xi^{d_{1} l_{1}^{2}\left(\mu_{i}+1\right)+d_{i} l_{i}\left(l_{1}-1\right)} \mu_{i}$ is nilpotent on $M_{(\mathbf{l} \mu+\kappa)}$, so $\left[\begin{array}{c}K_{i},-j l_{i} \\ l_{i}\end{array}\right]$ is invertible on $M_{(\mathbf{l} \mu+\kappa)}$ for $j \neq \mu_{i}$. Thus we get

$$
\left(\left[\begin{array}{c}
K_{i}, 0 \\
l_{i}
\end{array}\right]-\xi^{d_{l} l_{l}^{2}\left(\mu_{i}+1\right)+d_{l} l_{l}\left(l_{l}-1\right)} \mu_{i}\right) M_{(1 \mu+\kappa)}=0 .
$$

So $M_{(\mathbf{l} \mu+\kappa)}=M_{\mathbf{l} \mu+\kappa}$ since all $K_{i},\left[\begin{array}{c}K_{i}, 0 \\ l_{i}\end{array}\right]$ generate the algebra $U_{\xi}^{0}$. Therefore $M$ is isomorphic to $V_{\xi}(\mathbf{l} \mu+\kappa) \oplus V_{\xi}(\mathbf{l} \mu+\kappa)$.

(iii) Let $M_{1}$ be a submodule of $M$ such that $V_{\xi}(1 \mu+\kappa)$ is a quotient module of $M_{1}$. By (ii), $V_{\xi}(l \mu+\kappa)$ is isomorphic to a submodule of $M_{1}$. Since $M_{1}$ is a submodule of $M$, by (i), $M$ is isomorphic to $V_{\xi}(l \mu+\kappa) \oplus M^{\prime}$ for some 
submodule $M^{\prime}$ of $M$.

The lemma is proved.

3.5. Now we prove Theorem 3.2. Part (i) is a trivial consequence of Lemma 3.4 (iii).

(ii) According to part (i) and 3.3 (iii), for any finite dimensional $U_{\xi}$-module $M$ of type $\mathbb{1}$, the modules $V_{\xi}(\mathbb{l} \mu+\kappa) \otimes M$ and $M \otimes \mathbb{V}_{\xi}(\mathbb{l} \mu+\kappa)$ are projevtive and injective as well in the category $\mathscr{C}$. For any $\lambda$ in $\mathbb{Z}_{+}^{n}$, choose $v=(a, a, \ldots, a)$ in $\mathbb{Z}_{+}^{n}$ such that $\mathbb{1} v+\kappa-\lambda \in \mathbb{Z}_{+}^{n} . \quad$ By 3.3 (i) we have a nonzero $U_{\xi}$-homomorphism $V_{\xi}(\mathbb{1} v+\kappa) \rightarrow L_{\xi}(\lambda) \otimes V_{\xi}(\mathbb{l} v+\kappa-\lambda)$. By 3.3 (iii), this gives rise to a nonzero $U_{\xi}$-homomorphism $V_{\xi}(\mathbb{l} v+\kappa) \otimes V_{\xi}(\mathbb{l} v+\kappa-\lambda)^{\star} \rightarrow L_{\xi}(\lambda)$, which is necessarily surjective. Further, this surjective gives rise to a nonzero $U_{\xi}$-homomorphism $\quad L_{\xi}\left(-w_{0} \lambda\right) \simeq L_{\xi}(\lambda)^{*} \rightarrow\left(V_{\xi}(\mathbb{l} v+\kappa) \otimes V_{\xi}(\mathbb{l} v+\kappa-\lambda)^{*}\right)^{*} \simeq$ $V_{\xi}(\mathbb{\|}+\kappa) \otimes V_{\xi}(\mathbb{\|} v+\kappa-\lambda)$. (Note that $w_{0}(v)=-v$ for our choice.) Therefore the category $\mathscr{C}$ has enough injective objects and enough projective objects as well. Part (ii) is proved.

(iii) The $U_{\xi}$-modules $V_{\xi}(\mathbb{l} \mu+\kappa)^{*}, V_{\xi}(\mathbb{l} \mu+\kappa)^{\star}$ are isomorphic to $V_{\xi}(\mathbb{l} v+\kappa)$, where $v=-w_{0}(\mu)$. So for each $M \in \mathrm{ob} \mathscr{C}$, the modules $V_{\xi}(\mathbb{R} \mu+\kappa) \otimes M$ and $\left(V_{\xi}(\mathbb{l} \mu+\kappa) \otimes M\right)^{*}=V_{\xi}(\mathbb{\|}+\kappa) \otimes M^{*}$ are projective and injective as well in the category $\mathscr{C}$. By the proof of (ii) we see that (iii) is true.

(iv) We have seen that each indecomposable injective object is a direct summand of $V_{\xi}(\mathbb{l} v+\kappa) \otimes V_{\xi}(\delta)$ for some $v, \delta \in \mathbb{Z}_{+}^{n}$. So each injective object in ob $\mathscr{C}$ is an integrable $U_{\xi}$-module. Let $M$ be a finite dimensional $U_{\xi}$-module of type $\mathbb{1}$ and let $M^{\prime}$ be the maximal completely reducible submodule of $M$. By (ii), we can find an injective object $E$ in ob $\mathscr{C}$ and an injective $U_{\xi}$-homomorphism $M^{\prime} \hookrightarrow E$. Since $E$ is injective in the category, the above injection can be extended to an injective $U_{\xi}$-homomorphism $M G E$. Therefore $M$ is integrable since $E$ is integrable.

According to the statements in 3.1 we see that (v) is an immediate consequence of (iv).

(vi) It is no harm to assume that $E$ is indecomposable, then $E$ is a direct summand of $V:=V_{\xi}(\mathrm{l} v+\kappa) \otimes V_{\xi}(\delta)$ for some $v, \delta \in \mathbb{Z}_{+}^{n}$. By 3.3 (vii), $V$ has a submodule filtration $0=V_{h} \subset V_{h-1} \subset \cdots \subset V_{2} \subset V_{1}=V$ such that $V_{a} / V_{a+1}$ $\simeq V_{\xi}\left(\delta_{a}\right)$ for some $\delta_{a} \in \mathbb{Z}_{+}^{n}, a=1, \ldots, h-1$. Since $E$ is a direct summand of $V$, according to the following Lemma 3.6, the required filtration exists.

The theorem is proved.

Lemma 3.6. Let $M$ be a finite dimensional $U_{\xi}$-module of type $\mathbb{1}$. Assume that $M$ has a submodule filtration $0=M_{h} \subset M_{h-1} \subset \cdots \subset M_{2} \subset M_{1}=M$ such that $M_{a} / M_{a+1} \simeq V_{\xi}\left(\delta_{a}\right)$ for some $\delta_{a} \in \mathbb{Z}_{+}^{n}, a=1, \ldots, h-1$. (We say that $M$ has a filtration of Weyl modules.)

(i) Let $\lambda$ be a maximal weight of $M$ and $m$ be a non-zero element in $M_{\lambda}$. Then the submodule $U_{\xi} m$ of $M$ generated by $m$ is isomorphic to $\mathbb{V}_{\xi}(\lambda)$. 
(ii) Let $M^{\prime}, M^{\prime \prime}$ be two $U_{\xi}$-modules. Assume that $M$ is isomorphic to $M^{\prime} \oplus M^{\prime \prime}$, then both $M^{\prime}$ and $M^{\prime \prime}$ have filtrations of Weyl modules.

Proof. We copy the arguments in [J3, 3.5 and 3.6, pp. 279-280].

(i) Choose $k$ such that $m$ is in $M_{k}$ but not in $M_{k-1}$. Then the image $\bar{m}$ in $M_{k} / M_{k-1} \simeq V_{\xi}\left(\delta_{k}\right)$ of $m$ is not zero. Since $\lambda$ is a maximal weight of $M$, we necessarily have $\delta_{k}=\lambda$. Thus we get a surjective $U_{\xi}$-homomorphism $U_{\xi} m \rightarrow$ $V_{\xi}(\lambda), m \rightarrow \bar{m}$. According to 3.3 (i), the homomorphism is an isomorphism.

(ii) Choose a maximal weight $\lambda$ of $M$. It is no harm to assume that $M_{\lambda}^{\prime} \neq 0$. Let $m$ be a non-zero element in $M_{\lambda}^{\prime}$. By (i), $U_{\xi} m$ is isomorphic to $V_{\xi}(\lambda)$. By the argument in (i), the module $M / U_{\xi} m$ has a filtration of Weyl modules. But $M / U_{\xi} m$ is isomorphic to $\left(M^{\prime} / U_{\xi} m\right) \oplus M^{\prime \prime}$. Using induction on $\operatorname{dim}_{\mathbf{Q}(\xi)} M$ we see that both $M^{\prime}$ and $M^{\prime \prime}$ have filtrations of Weyl modules.

The lemma is proved.

Another main result of the section is the following.

Theorem 3.7. Let $\lambda \in \mathbf{Z}_{+, 1}^{n}, \mu \in \mathbf{Z}_{+}^{n}$. Then

(i) The module $V_{\xi}(1 \mu+\kappa+\lambda)$ contains a unique irreducible submodule.

(ii) The irreducible submodule of $V_{\xi}(\mathbf{l} \mu+\kappa+\lambda)$ has highest weight $\mathbf{l} \mu+\kappa+w_{0} \lambda$ and is generated by $y_{\lambda}^{\prime} v_{1 \mu+\kappa+\lambda}$. (See Corollary 2.7 (ii) for the definition of $y_{\lambda}^{\prime}$.)

Proof. (i) In the proof of Theorem 3.5 (ii) we have seen that $V_{\xi}(1 \mu+\kappa) \otimes V_{\xi}(\lambda)$ is an injective object in the category $\mathscr{C}$. According to 3.3 (vii) and Lemma 3.6 (i), the submodule of $V_{\xi}(1 \mu+\kappa) \otimes V_{\xi}(\lambda)$ generated by $v_{\mathbf{l} \mu+\kappa} \otimes v_{\lambda}$ is isomorphic to $V_{\xi}(1 \mu+\kappa+\lambda)$. Let $E$ be the indecomposable direct summand of $V_{\xi}(\mathbf{l} \mu+\kappa) \otimes V_{\xi}(\lambda)$ containing $v_{\mathbf{l} \mu+\kappa} \otimes v_{\lambda}$, then $V_{\xi}(\mathbf{l} \mu+\kappa+\lambda)$ is isomorphic to a submodule of $E$. The module $E$ contains a unique irreducible submodule since $E$ is an indecomposable injective object in the category $\mathscr{C}$. This forces that $V_{\xi}(\mathbf{l} \mu+\kappa+\lambda)$ contains a unique irreducible submodule.

(ii) We need to prove that

(a) The element $y_{\lambda}^{\prime} v_{\mathbf{1} \mu+\kappa+\lambda}$ is a primitive element in $V_{\xi}(\mathbf{l} \mu+\kappa+\lambda)$.

(b) The element $y_{\lambda}^{\prime} v_{1 \mu+\kappa+\lambda}$ generates an irreducible submodule of $V_{\xi}(1 \mu+$ $\kappa+\lambda)$.

By Prop. 2.8 (ii), $F_{\kappa} v_{\kappa} \neq 0$, so $F_{\kappa} \notin I_{\kappa}^{-}$. But $I_{\kappa+\lambda}^{-} \subseteq I_{\kappa}^{-}$. Hence $F_{\kappa} \notin I_{\kappa+\lambda}^{-}$. This implies that in $V_{\xi}(\kappa+\lambda)$ we have $F_{\kappa} v_{\kappa+\lambda} \neq 0$. Since $F_{\alpha} F_{\kappa} \in \mathbf{u}_{\xi}^{-}$if $\alpha \in R_{l}^{+}$ and $F_{\kappa}$ has the maximal degree in $\mathbf{u}_{\xi}^{-}$(recall the definition of degree in 2.3), we see $F_{\alpha} F_{\kappa}=0$ in $U_{\xi}$ for all $\alpha \in R_{l}^{+}$. In particular, $F_{\alpha} F_{\kappa} v_{\kappa+\lambda}=0$ for all $\alpha \in R_{l}^{+}$. Noting that $F_{\kappa}$ has degree $2 \kappa$ and $\xi^{d_{1} l_{l}\left\langle\alpha_{1}^{\vee}, 2 \kappa\right\rangle}=1(i \in[1, n])$, by [L7, Theorem 35.4 .2 (a), p. 276], $F_{i}^{\left(l_{1}\right)} F_{\kappa}-F_{\kappa} F_{i}^{\left(l_{1}\right)} \in \mathbf{u}_{\xi}^{-}$. But $F_{i}^{\left(l_{1}\right)} F_{\kappa}-F_{\kappa} F_{i}^{\left(l_{l}\right)}$ has degree $2 \kappa+l_{i} \alpha_{i}>2 \kappa$, so $F_{i}^{\left(l_{l}\right)} F_{\kappa}=F_{\kappa} F_{i}^{\left(l_{l}\right)}$. Since $F_{\kappa}=x F_{i}^{\left(l_{i}-1\right)}$ for some $x$ in $\mathbf{u}_{\xi}^{-}$(see Lemma 2.5), we get $F_{i}^{\left(l_{l}\right)} F_{\kappa}=F_{\kappa} F_{i}^{\left(l_{l}\right)}=x F_{i}^{\left(l_{l}-1\right)} F_{i}^{\left(l_{2}\right)}=x F_{i}^{\left(2 l_{2}-1\right)}$ for $i=1,2, \ldots, n$ (cf. [L2, 3.2 (c), p. 62]). Thus $F_{i}^{\left(l_{2}\right)} F_{\kappa} v_{\kappa+\lambda}=x F_{i}^{\left(2 l_{1}-1\right)} v_{\kappa+\lambda}=0$ for $i=1,2, \ldots, n$. Therefore, $-\kappa+\lambda$ (the weight of $F_{\kappa} v_{\kappa+\lambda}$ ) is the lowest weight 
of the submodule $M^{\prime}$ of $V_{\xi}(\kappa+\lambda)$ generated by $F_{\kappa} v_{\kappa+\lambda}$.

By the proof of Prop. 2.6 (ii), the submodule $M^{\prime}$ of $V_{\xi}(\kappa+\lambda)$ is an irreducible module of highest weight $\kappa+w_{0} \lambda$. By Corollary 2.7 (ii), the irreducible module $M^{\prime}$ is generated by $y_{\lambda}^{\prime} v_{\kappa+\lambda}$. Thus

(c) $y_{\lambda}^{\prime} v_{\kappa+\lambda}$ is a primitive element in $V_{\xi}(\kappa+\lambda)$ (since it has weight $\kappa+w_{0} \lambda$ ) and generates the irreducible submodule of $V_{\xi}(\kappa+\lambda)$.

According to the proof of Theorem 3.2 (ii), $M:=V_{\xi}(\kappa) \otimes V_{\xi}(\lambda) \otimes L_{\xi}(\mathbb{l} \mu)$ is injective in $\mathscr{C}$. By Theorem 3.2 (vi) and Lemma 3.6 (i), the submodule $N$ of $M$ generated by $v_{\kappa} \otimes v_{\lambda} \otimes \bar{v}_{1 \mu}$ is isomorphic to $V_{\xi}(\mathbb{1} \mu+\kappa+\lambda)$. Since the submodule of $V_{\xi}(\kappa) \otimes V_{\xi}(\lambda)$ generated by $v_{\kappa} \otimes v_{\lambda}$ is isomorphic to $V_{\xi}(\kappa+\lambda)$ (see 3.3 (vii) and Lemma 3.6 (i)), by (c) and 2.2 (v), we get

(d) The element $m^{\prime}:=y_{\lambda}^{\prime}\left(v_{\kappa} \otimes v_{\lambda} \otimes \bar{v}_{1 \mu}\right)=\left(y_{\lambda}^{\prime}\left(v_{\kappa} \otimes v_{\lambda}\right)\right) \otimes \bar{v}_{1 \mu}$ is primitive in $N$.

Since $\kappa+w_{0} \lambda$ is I-restricted, by (c), (d) and 2.2 (iv), we see that $m^{\prime}$ generates an irreducible submodule of $N$. This completes the proof of (ii).

\section{§4. The Elements $\boldsymbol{x}_{\lambda}^{\prime}$}

4.1. Recall that in 1.4 we have defined the element $x_{\lambda}^{\prime} \in U_{\xi}^{-}$and in Corollary 2.7 (ii) defined the element $y_{\lambda}^{\prime} \in \mathbf{u}_{\xi}^{-}$for every $\lambda$ in $\mathbb{Z}_{+}^{n}$. The main result of this section is Theorem 4.2. We prove it after establishing several lemmas. It is a pity that the author could not find a simple proof of Theorem 4.2 except for type $A_{n}, B_{2}$ and could not prove it for type $G_{2}$. For convenience we say that the quantized hyperalgebra $U_{\xi}$ has no factors of type $G_{2}$ if any indecomposable component of the Cartan matrix $\left(a_{i j}\right)$ is not of type $G_{2}$.

Theorem 4.2. Assume that $U_{\xi}$ has no factors of type $G_{2}$. Let $\lambda \in \mathbb{Z}_{+, 1}^{n}$, $\mu \in \mathbf{Z}_{+}^{n}$. Then

(i) The element $x_{\lambda}^{\prime} v_{1 \mu+\kappa+\lambda}$ is a primitive element in $V_{\xi}(1 \mu+\kappa+\lambda)$.

(ii) We have $x_{\lambda}^{\prime}=\eta y_{\lambda}^{\prime}$ for some nonzero number $\eta \in \mathbb{Q}(\xi)$. In particular, $x_{\lambda}^{\prime}$ is in $\mathbf{u}_{\xi}^{-}$.

Lemma 4.3. Let $M$ be an integrable $U_{\xi}$-module of type $\mathbb{1}$ and let $m \in M_{\mu}\left(\mu \in \mathbb{Z}^{n}\right)$. Let $i, j$ be integers in $[1, n]$ and let $a, b, c$ be non-negative integers.

(i) Assume that $E_{i}^{(h)} m=0$ for $h \geq 1$. Then $F_{i}^{(a)} F_{j}^{(b)} F_{i}^{(c)} m=0$ if $a+\left\langle\alpha_{j}, \alpha_{i}^{\vee}\right\rangle b$ $+c\rangle\left\langle\mu, \alpha_{i}^{\vee}\right\rangle$. In particular, $F_{i}^{(a)} F_{j}^{(b)} m=0$ if $\left.a+\left\langle\alpha_{j}, \alpha_{i}^{\vee}\right\rangle b\right\rangle\left\langle\mu, \alpha_{i}^{\vee}\right\rangle$.

(ii) Assume that $E_{i}^{(h)} m=0, E_{j}^{(h)} m=0$ for $h \geq 1$. Then $F_{i}^{(a)} E_{j}^{(b)} F_{j}^{(c)} m=0$ if $a+$ $\left.\left\langle\alpha_{j}, \alpha_{i}^{\vee}\right\rangle(c-b)\right\rangle\left\langle\mu, \alpha_{i}^{\vee}\right\rangle$.

Proof. (i) By the commutation relations in [L4, 5.3-5.4, pp.95-97], the element $F_{i}^{(a)} F_{j}^{(b)} F_{i}^{(c)}$ is in the left ideal of $U_{\xi}^{-}$generated by $F_{i}^{(h)}, h \geq a+$ $\left.\left\langle\alpha_{j}, \alpha_{i}^{\vee}\right\rangle b+c\right\rangle\left\langle\mu, \alpha_{i}^{\vee}\right\rangle$. Now applying 3.3 (i) to the subalgebra of $U_{\xi}$ generated by the elements $E_{i}^{(h)}, F_{i}^{(h)}, K_{i}, K_{i}^{-1}(h \geq 0)$, we see that (i) is true. 
(ii) If $b>c$, then

$$
F_{i}^{(a)} E_{j}^{(b)} F_{j}^{(c)} m=F_{i}^{(a)} \sum_{0 \leq h \leq c} F_{j}^{(c-h)}\left[\begin{array}{c}
K_{j}, 2 h-c-b \\
h
\end{array}\right] E_{j}^{(b-h)} m=0 .
$$

If $b \leq c$, using (i), we see

$$
F_{i}^{(a)} E_{j}^{(b)} F_{j}^{(c)} m=F_{i}^{(a)} F_{j}^{(c-b)}\left[\begin{array}{c}
K_{j}, b-c \\
b
\end{array}\right] m=\left[\begin{array}{c}
\left\langle\mu, \alpha_{i}^{\vee}\right\rangle+b-c \\
b
\end{array}\right]_{d_{j}, \xi} F_{i}^{(a)} F_{j}^{(c-b)} m=0 .
$$

The lemma is proved.

Lemma 4.4. Let $\lambda \in \mathbf{Z}_{+, 1}^{n}, \mu \in \mathbf{Z}_{+}^{n}, w \in W$. Then

(i) In $V_{\xi}(\mathbf{l} \mu+\kappa+\lambda)$ we have $x_{\lambda, w}^{\prime} v_{1 \mu+\kappa+\lambda} \neq 0$.

(ii) If $l_{i} \geq 2$, then $E_{i} x_{\lambda, w}^{\prime} v_{1 \mu+\kappa+\lambda}=0$.

(iii) If $l_{\alpha} \geq 2$, then $E_{\alpha} x_{\lambda, w}^{\prime} v_{1 \mu+\kappa+\lambda}=0$.

(iv) Assume that $x_{\lambda, w}^{\prime}=F_{i_{1}}^{\left(a_{1}\right)} F_{i_{2}}^{\left(a_{2}\right)} \cdots F_{i_{k}}^{\left(a_{k}\right)}$. Given non negative integers $b_{1}$, $b_{2}, \ldots, b_{k}$, if $a_{h}-b_{h} \in l_{i_{h}} \mathbf{Z}$ for $h=1, \ldots, k$, then $E_{i} F_{i_{1}}^{\left(b_{1}\right)} F_{i_{2}}^{\left(b_{2}\right)} \cdots F_{i_{k}}^{\left(b_{k}\right)} v_{\mathbf{l}_{\mu+\kappa+\lambda}}=0$ when $l_{i} \geq 2$.

Proof. Set $m:=v_{\mathbf{l} \mu+\kappa+\lambda}$.

(i) According to $\left[\mathrm{L} 7\right.$, Lemma 39.1 .2$, p. 304], in $V_{\xi}(\lambda)$ we have $x_{\lambda, w}^{\prime} v_{\lambda} \neq 0$. By 1.2 (i), this implies that $x_{\lambda, w}^{\prime} m \neq 0$.

(ii) According to $\left[\mathrm{L} 7\right.$, Lemma 39.1.4, p.305], there exists $z$ in $U_{\xi}^{-}$such that

$$
E_{i} x_{\lambda, w}^{\prime}=x_{\lambda, w}^{\prime} E_{i}+z\left[\begin{array}{c}
K_{i}, 1-\left\langle\lambda, \alpha_{i}^{\vee}\right\rangle \\
1
\end{array}\right]
$$

Since $\quad l_{i} \geq 2, \quad\left[\begin{array}{c}K_{i}, 1-\left\langle\lambda, \alpha_{i}^{\vee}\right\rangle \\ 1\end{array}\right] m=\left[\begin{array}{c}l_{i}\left\langle\mu, \alpha_{i}^{\vee}\right\rangle+l_{i} \\ 1\end{array}\right]_{d_{1} . \xi^{z}} m=0 . \quad$ Therefore $E_{i} x_{\lambda, w}^{\prime} m=0$.

(iii) It is no harm to assume that the Cartan matrix $\left(a_{i j}\right)$ is indecomposable. When $\mathbf{u}_{\xi}^{-}$is generated by the elements $1, F_{i}(i=1,2, \ldots, n)$, the assertion is a simple consequence of (ii). When $\mathbf{u}_{\xi}^{-}$is not generated by the elements $1, F_{i}(i=1,2, \ldots, n)$, one of the following cases must happen,

(a) The Cartan matrix $\left(a_{i j}\right)$ is of type $B_{n}, C_{n}, F_{4}$ and $l=4$.

(b) The Cartan matrix $\left(a_{i j}\right)$ is of type $G_{2}$ and $l=3,4,6$.

The generators of $\mathbf{u}_{\xi}^{-}$are described in [L4, 8.3, pp. 107-108] explicitly. Using induction on the height of $\alpha$ and using Theorem 6.6 in [L4, pp. 103-104], one can prove that $E_{\alpha} F_{i}^{(a)}-F_{i}^{(a)} E_{\alpha} \in U_{\xi}^{-} \mathbf{u}_{\xi}^{0} \mathbf{u}_{\xi}^{+}$for all $i \in[1, n]$, $a \in \mathbf{N}$. Using (ii), we then can prove (iii) by induction on $l(w)$ and on height of $\alpha$.

Part (iv) is a simple consequence of (ii).

Lemma 4.5. Let $\lambda \in \mathbb{Z}_{+, 1}^{n}, w \in W$. Assume that the Cartan matrix $\left(a_{i j}\right)$ is 
symmetric. If $s_{j} w \geq w$, then $E_{j}^{(a)} x_{\lambda, w}^{\prime} v_{\kappa+\lambda}=0$ for all $a \geq 1$. (We also use " $\geq$ " for the Bruhat order on $W$.)

Proof. Set $m:=v_{\kappa+\lambda}$. Noting that all $l_{1}, l_{2}, \ldots, l_{n}$ are equal, we simply write $l^{\prime}$ for any one of them. Since $U_{\xi}^{+}$is generated by the elements $E_{i}, E_{i}^{\left(l^{\prime}\right)}$ for $i=1,2, \ldots, n$, [L2, Prop. 3.2 (b), p. 62], by Lemma 4.4 (ii), it suffices to prove that $E_{j}^{\left(l^{\prime}\right)} x_{\lambda, w}^{\prime} m=0$. We use induction on the length $l(w)$ of $w$. Let $s_{i_{1}} s_{i_{2}} \cdots s_{i_{k}}$ be a reduced expression of $w$. We shall write $a_{h}$ instead of $\left\langle s_{i_{h+1}} \cdots s_{i_{k}} \lambda, \alpha_{i_{h}}^{v}\right\rangle$ for $h=1, \ldots, k$. When $k=0,1$, nothing need to be proved. Now assume that $k \geq 2$. Set $i:=i_{1}$ and let $u$ be the shortest element of the coset $\left\langle s_{i}, s_{j}\right\rangle w$ (here $\left\langle s_{i}, s_{j}\right\rangle$ denotes the subgroup of $W$ generated by $\left.s_{i}, s_{j}\right)$. Since the Cartan matrix is symmetric, $k-1 \geq l(u) \geq k-2$.

If $l(u)=k-1$, then $u=s_{i_{2}} \cdots s_{i_{k}}$ and $s_{j} u \geq u$. Note that $i \neq j$, using induction hypothesis, we see $E_{j}^{\left(l^{\prime}\right)} x_{\lambda, w}^{\prime} m=F_{i}^{\left(a_{1}\right)} E_{j}^{\left(l^{\prime}\right)} x_{\lambda, u}^{\prime} m=0$.

If $l(u)=k-2$, we may assume that $i_{2}=j$ and $u=s_{i_{3}} \cdots s_{i_{k}}$. Then $s_{i} u \geq u$, $s_{j} u \geq u$ and $E_{i}^{(a)} x_{\lambda, u}^{\prime} m=0, \quad E_{j}^{(a)} x_{\lambda, u}^{\prime} m=0$ for all $a \geq 1$. So $E_{j}^{\left(l^{\prime}\right)} x_{\lambda, w}^{\prime} m=$ $F_{i}^{\left(a_{1}\right)} E_{j}^{\left(l^{\prime}\right)} F_{j}^{\left(a_{2}\right)} x_{\lambda, u}^{\prime} m$. Noting that $a_{1}=\left\langle s_{j} u \lambda, \alpha_{i}^{\vee}\right\rangle=\left\langle u \lambda, \alpha_{i}^{\vee}+\alpha_{j}^{\vee}\right\rangle=\left\langle u \lambda, \alpha_{i}^{\vee}\right\rangle$ $+a_{2}$ and $x_{\lambda, u}^{\prime} m$ has weight $\kappa+u \lambda$, by Lemma 4.3 (ii) we see $E_{j}^{\left(l^{\prime}\right)} x_{\lambda, w}^{\prime} m=0$.

The lemma is proved.

Lemma 4.6. Let $\lambda \in \mathbb{Z}_{+, 1}^{n}$. Assume that $U_{\xi}$ has no factors of type $G_{2}$. Then in $V_{\xi}(\kappa+\lambda)$ the element $x_{\lambda}^{\prime} v_{\kappa+\lambda}$ is primitive.

Proof. Set $m:=v_{\kappa+\lambda}$. Since $U_{\xi}^{+}$is generated by the elements $E_{i}, E_{i}^{\left(l_{l}\right)}$ for $i=1,2, \ldots, n$, by Lemma 4.4 (ii), it suffices to prove that $E_{i}^{\left(l_{l}\right)} x_{\lambda}^{\prime} m=0$ for all $i$. Set $\lambda_{i}=\left\langle\lambda, \alpha_{i}^{\vee}\right\rangle$ for $i=1,2, \ldots, n$.

(a) Assume that $\left(a_{i j}\right)$ is symmetric. Choose a reduced expression $s_{i_{1}} s_{i_{2}} \cdots s_{i_{r}}$ of the longest element $w_{0}$ of $W$ such that $i_{1}=i$. Note that $a:=\left\langle s_{i_{2}} \cdots s_{i_{r}} \lambda, \alpha_{i}^{\vee}\right\rangle<l_{i}$, so

$$
E_{i}^{\left(l_{2}\right)} x_{\lambda}^{\prime} m=\sum_{0 \leq h \leq a} F_{i}^{(a-h)}\left[\begin{array}{c}
K_{i}, 2 h-a-l_{i} \\
h
\end{array}\right] E_{i}^{\left(l_{l}-h\right)} x_{\lambda, u}^{\prime} m,
$$

where $u=s_{i_{2}} \cdots s_{i_{r}}$. By Lemma 4.5, $E_{i}^{\left(l_{x}-h\right)} x_{\lambda, u}^{\prime} m=0$ for $h=0,1, \ldots, a$. Therefore $E_{i}^{\left(l_{2}\right)} x_{\lambda}^{\prime} m=0$ for $i=1,2, \ldots, n$.

(b) Assume that $\left(a_{i j}\right)$ is of type $B_{n}$. We number the simple roots in $R^{+}$ so that $\left\langle\alpha_{2}, \alpha_{1}^{v}\right\rangle=-2,\left\langle\alpha_{1}, \alpha_{2}^{v}\right\rangle=\left\langle\alpha_{2}, \alpha_{3}^{v}\right\rangle=\cdots=\left\langle\alpha_{n-1}, \alpha_{n}^{v}\right\rangle=-1$. We have $d_{1}=1, d_{2}=\cdots=d_{n}=2, l_{2}=\cdots=l_{n}$, and $2 l_{j} \geq l_{1} \geq l_{j}$ for $j=2, \ldots, n$. We use induction on $n$.

When $n=2$, write $a:=\left\langle\lambda, \alpha_{1}^{\vee}\right\rangle, b:=\left\langle\lambda, \alpha_{2}^{\vee}\right\rangle$. Then

$$
x_{\lambda}^{\prime}=F_{1}^{(a)} F_{2}^{(a+b)} F_{1}^{(a+2 b)} F_{2}^{(b)}=F_{2}^{(b)} F_{1}^{(a+2 b)} F_{2}^{(a+b)} F_{1}^{(a)} .
$$

Since $l_{1}>a$, using Lemma 4.4 (ii) we see

$$
E_{1}^{\left(l_{1}\right)} x_{\lambda}^{\prime} m=F_{1}^{(a)} F_{2}^{(a+b)} E_{1}^{\left(l_{1}\right)} F_{1}^{(a+2 b)} F_{2}^{(b)} m .
$$


Note that $F_{2}^{(b)} m$ is a primitive element of weight $\kappa+\lambda-b \alpha_{2}$. Now

$$
\left.a+b-\left\langle\alpha_{1}, \alpha_{2}^{\vee}\right\rangle\left(l_{1}-a-2 b\right)=l_{1}-b\right\rangle l_{2}-1-b=\left\langle\kappa+\lambda-b \alpha_{2}, \alpha_{2}^{\vee}\right\rangle .
$$

By Lemma 4.3 (ii) we have $E_{1}^{\left(l_{1}\right)} x_{\lambda}^{\prime} m=0$. Similarly we have

$$
E_{2}^{\left(l_{2}\right)} x_{\lambda}^{\prime} m=F_{2}^{(b)} F_{1}^{(a+2 b)} E_{2}^{\left(l_{2}\right)} F_{2}^{(a+b)} F_{1}^{(a)} m=0 .
$$

Now suppose that the lemma is true for type $B_{n-1}$. Let $w$ be the longest element in $\left\langle s_{1}, s_{2}, \ldots, s_{n-1}\right\rangle$ (the subgroup of $W$ generated by $s_{1}, s_{2}, \ldots, s_{n-1}$ ). Then

$$
w_{0}=s_{n} s_{n-1} \cdots s_{2} s_{1} s_{2} \cdots s_{n-1} s_{n} w=w s_{n} s_{n-1} \cdots s_{2} s_{1} s_{2} \cdots s_{n-1} s_{n} .
$$

Set

$$
a_{h}:=\left\langle s_{h-1} \cdots s_{2} s_{1} s_{2} \cdots s_{n-1} s_{n} w \lambda, \alpha_{h}^{\vee}\right\rangle=\lambda_{h}+\lambda_{h+1}+\cdots+\lambda_{n}, \quad h=2, \ldots, n,
$$

and

$$
\begin{gathered}
b_{1}:=\left\langle s_{2} \cdots s_{n-1} s_{n} w \lambda, \alpha_{1}^{\vee}\right\rangle=\lambda_{1}+2 \lambda_{2}+\cdots+2 \lambda_{n}, \\
b_{2}:=\left\langle s_{3} \cdots s_{n-1} s_{n} w \lambda, \alpha_{2}^{\vee}\right\rangle=\lambda_{1}+\lambda_{2}+\cdots+\lambda_{n}, \\
b_{h}:=\left\langle s_{h+1} \cdots s_{n-1} s_{n} w \lambda, \alpha_{h}^{\vee}\right\rangle=\lambda_{1}+\lambda_{2}+\cdots+\lambda_{n}+\lambda_{2}+\cdots+\lambda_{h-1}, \quad h=3, \ldots, n .
\end{gathered}
$$

Then we have

(b1) $x_{\lambda}^{\prime}=F_{n}^{\left(a_{n}\right)} \cdots F_{2}^{\left(a_{2}\right)} F_{1}^{\left(b_{1}\right)} F_{2}^{\left(b_{2}\right)} \cdots F_{n}^{\left(b_{n}\right)} x_{\lambda, w}^{\prime}$.

By induction hypothesis, we have

(b2) The element $x_{\lambda, w}^{\prime} m$ is primitive in $V_{\xi}(\kappa+\lambda)$ of weight $\kappa+w \lambda=\left(l_{1}-1-\right.$ $\left.\lambda_{1}, l_{2}-1-\lambda_{2}, \ldots, l_{n-1}-1-\lambda_{n-1}, l_{n}-1+b_{n}\right)$.

Using Lemma 4.3 (ii) and Lemma 4.4 (ii) repeatedly we see

(b3) $E_{i}^{\left(l_{1}\right)} F_{h}^{\left(b_{h}\right)} F_{h+1}^{\left(b_{h}+1\right)} \cdots F_{n}^{\left(b_{n}\right)} x_{\lambda, w}^{\prime} m=0$ for $h=1,2, \ldots, n$ and $i \neq h$.

(b4) $E_{i}^{\left(l_{1}\right)} F_{h}^{\left(a_{h}\right)} \cdots F_{2}^{\left(a_{2}\right)} F_{1}^{\left(b_{1}\right)} F_{2}^{\left(b_{2}\right)} \cdots F_{n}^{\left(b_{n}\right)} x_{\lambda, w}^{\prime} m=0$ for $i, h=2, \ldots, n$ and $i \neq h$.

Since $a_{n}=\lambda_{n}<l_{n}$, by (b4) and Lemma 4.4 (ii) we know that $E_{i}^{\left(l_{2}\right)} x_{\lambda}^{\prime} m=0$ for $i=2, \ldots, n$.

We need to do a little more to see that $E_{1}^{\left(l_{1}\right)} x_{\lambda}^{\prime} m=0$. Let $u$ be the longest element of $\left\langle s_{2}, \ldots, s_{n}\right\rangle$. Then

$$
w_{0}=s_{1} s_{2} s_{1} s_{3} s_{2} s_{1} \cdots s_{n} \cdots s_{2} s_{1} u .
$$

For $n \geq i \geq j \geq 1$, set

$$
\begin{aligned}
c_{i j} & :=\left\langle s_{j-1} \cdots s_{1} s_{i+1} \cdots s_{1} s_{i+2} \cdots s_{1} \cdots s_{n} \cdots s_{2} s_{1} u \lambda, \alpha_{j}^{\vee}\right\rangle \\
& =\lambda_{1}+\cdots+\lambda_{i}+\lambda_{j+1}+\cdots+\lambda_{i} .
\end{aligned}
$$

(Convention: $\lambda_{i+1}+\cdots+\lambda_{i}=0$ ). Then we have

(b5) $x_{\lambda}^{\prime}=F_{1}^{\left(c_{11}\right)} F_{2}^{\left(c_{22}\right)} F_{1}^{\left(c_{21}\right)} \cdots F_{n}^{\left(c_{n n}\right)} \cdots F_{1}^{\left(c_{n 1}\right)} x_{\lambda, u}^{\prime}$. 
By (a), we have

(b6) The element $x_{\lambda, u}^{\prime} m$ is primitive in $V_{\xi}(\kappa+\lambda)$ of weight $\kappa+u \lambda=\left(l_{1}-1+\right.$ $\left.c_{n 1}, l_{2}-1-\lambda_{n}, \ldots, l_{n}-1-\lambda_{2}\right)$.

Note that $c_{11}=\lambda_{1}<l_{1}$. Now we can see $E_{1}^{\left(l_{1}\right)} x_{\lambda}^{\prime} m=0$ by repeatedly using Lemma 4.3 (ii) and Lemma 4.4 (ii). Thus we complete the proof type $B_{n}$.

(c) Similarly, we prove the lemma for type $C_{n}$.

(d) Similarly we prove the lemma for type $F_{4}$ but need a little patience. We number the simple roots so that $\left\langle\alpha_{i}, \alpha_{i+1}^{v}\right\rangle=-1$ for $i=1,2,3$. Then $d_{1}=d_{2}=1, d_{3}=d_{4}=2$. Moreover, $\alpha_{1}, \alpha_{2}, \alpha_{3}$ generate a root system of type $C_{3}$ and $\alpha_{2}, \alpha_{3}, \alpha_{4}$ generate a root system of type $B_{3}$. Let $w$ be the longest element of the group $\left\langle s_{1}, s_{2}, s_{3}\right\rangle$. Then the longest element of $W$ is $s_{4} s_{3} s_{2} s_{3} s_{4} s_{1} s_{2} s_{3} s_{4} s_{2} s_{3} s_{1} s_{2} s_{3} s_{4} w$. We shall also write $\lambda_{a, b, c, d}$ for $a \lambda_{1}+b \lambda_{2}+c \lambda_{3}+d \lambda_{4}$. For non-negative integers $\theta_{1}, \ldots, \theta_{4}$, define

$$
\begin{aligned}
& Y_{1}\left(\theta_{1}, \ldots, \theta_{4}\right) \\
& \quad:=F_{4}^{\left(\lambda_{4}\right)} F_{3}^{\left(\lambda_{3}+\lambda_{4}-\theta_{1} l_{3}\right)} F_{2}^{\left(\lambda_{2}+2 \lambda_{3}+2 \lambda_{4}-\theta_{2} l_{2}\right)} F_{3}^{\left(\lambda_{2}+\lambda_{3}+\lambda_{4}-\theta_{3} l_{3}\right)} F_{4}^{\left(\lambda_{2}+2 \lambda_{3}+\lambda_{4}-\theta_{4} l_{4}\right)}, \\
& Y_{2}\left(\theta_{1}, \ldots, \theta_{4}\right):=F_{1}^{\left(\lambda_{1,1,2,2}-\theta_{1} l_{1}\right)} F_{2}^{\left(\lambda_{1,2,2,2}-\theta_{2} l_{2}\right)} F_{3}^{\left(\lambda_{1,2,3,2}-\theta_{3} l_{3}\right)} F_{4}^{\left(\lambda_{1,1,1,1}-\theta_{4} l_{4}\right)}, \\
& Y_{3}\left(\theta_{2}, \theta_{3}\right):=F_{2}^{\left(\lambda_{1,2,4,2}-\theta_{2} l_{2}\right)} F_{3}^{\left(\lambda_{1,1,2,1}-\theta_{3} l_{3}\right)}, \\
& Y_{4}\left(\theta_{1}, \ldots, \theta_{4}\right):=F_{1}^{\left(\lambda_{1,3,4,2}-\theta_{1} l_{1}\right)} F_{2}^{\left(\lambda_{2,3,4,2}-\theta_{2} l_{2}\right)} F_{3}^{\left(\lambda_{1,2,2,1}-\theta_{3} l_{3}\right)} F_{4}^{\left(\lambda_{1,2,3,1}-\theta_{4} l_{4}\right)} .
\end{aligned}
$$

We simply write $Y_{i}$ for $Y_{i}(0, \ldots, 0), i=1,2,3,4$.

Then we have

(d1) $x_{\lambda}^{\prime}=Y_{1} Y_{2} Y_{3} Y_{4} x_{\lambda, w}^{\prime}$.

According to (c), we get

(d2) The element $m^{\prime}:=x_{\lambda, w}^{\prime} m$ is primitive in $V_{\xi}(\kappa+\lambda)$ and has weight $\left(l_{1}-1-\lambda_{1}, l_{2}-1-\lambda_{2}, l_{3}-1-\lambda_{3}, l_{4}-1+\lambda_{1,2,3,1}\right)$.

Using (d2), Lemma 4.3 (ii) and Lemma 4.4 (ii) repeatedly, step by step, we obtain

(d3) $\quad E_{i}^{\left(l_{2}\right)} Y_{4} x_{\lambda, w}^{\prime} m$ for $i=2,3,4$.

(d4) $E_{i}^{\left(l_{2}\right)} Y_{3} Y_{4} x_{\lambda, w}^{\prime} m=0$ for $i=1,3,4$.

(d5) $E_{2}^{\left(l_{2}\right)} Y_{2} Y_{3} Y_{4} x_{\lambda, w}^{\prime} m=0$.

(d*) $\quad E_{1}^{\left(l_{1}\right)} Y_{1} Y_{2} Y_{3} Y_{4} x_{\lambda, w}^{\prime} m=0$.

To avoid more troubles we use the following consequence of Theorem 4.2 for type $B_{3}$ (cf. Corollary 4.10 (ii)).

(d6) $Y_{1}(0,1,0,0) F_{2}^{\left(\lambda_{2}\right)} F_{3}^{\left(\lambda_{2}+\lambda_{3}\right)} F_{2}^{\left(\lambda_{2}+2 \lambda_{3}\right)} F_{3}^{\left(\lambda_{3}\right)}=0$.

Obviously, (d6) implies the following

(d7) $Y_{1}(0,1,0,0) Y_{2} Y_{3} Y_{4} x_{\lambda, w}^{\prime}=0$.

Combining (d5) and (d7), also using Lemma 4.4 (ii) we see (d $\star)_{2}^{\left(l_{2}\right)} Y_{1} Y_{2} Y_{3} Y_{4} x_{\lambda, w}^{\prime} m=0$.

Let $u$ be the longest element of $\left\langle s_{2}, s_{3}, s_{4}\right\rangle$. We may write down a 
presentation for $x_{\lambda}^{\prime}$ according to the reduced expression $s_{1} s_{2} s_{3} s_{2} s_{1} s_{4} s_{3} s_{2} s_{1} s_{3} s_{2}$ $s_{4} s_{3} s_{2} s_{1} u$. Using an argument similar to that for $(\mathrm{d} *)$ and $(\mathrm{d} \star)$ we obtain (d†) $E_{i}^{\left(l_{2}\right)} Y_{1} Y_{2} Y_{3} Y_{4} x_{\lambda, w}^{\prime} m=0$ for $i=3,4$.

The lemma is proved for type $F_{4}$ and the proof is completed.

Lemma 4.7. Assume that $\left(a_{i j}\right)$ is symmetric and $\lambda \in \mathbf{Z}_{+}^{n}$ is l-restricted. Let $s_{i_{1}} s_{i_{2}} \cdots s_{i_{r}}$ be a reduced expression of the longest element $w_{0}$. Set $a_{h}:=$ $\left\langle s_{i_{h+1}} s_{i_{h+2}} \cdots s_{i_{r}} \lambda, \alpha_{i_{h}}^{\vee}\right\rangle$ for $h=1,2, \ldots, r$. Given non-negative integers $\theta_{1}, \theta_{2}, \ldots$, $\theta_{r}$, we define

$$
Y_{\mathbf{i}}\left(\theta_{1}, \ldots, \theta_{r}\right):=F_{i_{1}}^{\left(a_{1}-\theta_{1} l^{\prime}\right)} F_{i_{2}}^{\left(a_{2}-\theta_{2} l^{\prime}\right)} \ldots F_{i_{r}}^{\left(a_{r}-\theta_{r} l^{\prime}\right)} .
$$

Then in $V_{\xi}(\kappa+\lambda)$ we have $Y_{\mathbf{i}}\left(\theta_{1}, \ldots, \theta_{r}\right) v_{\kappa+\lambda}=0$ if $\theta_{1}+\cdots+\theta_{r} \geq 1$. Here $\mathbf{i}=\left(i_{1}, i_{2}, \ldots, i_{r}\right)$ and $l^{\prime}:=l_{1}=\cdots=l_{r} . \quad\left(\right.$ Recall that $F_{i}^{(a)}=0$ for all $i$ and $a<0$, see 1.1.)

Proof. We use induction on $h$ to prove that

(a) $Y_{\mathbf{i}}\left(\theta_{1}, \ldots, \theta_{h}, 0, \ldots, 0\right) v_{\kappa+\lambda}=0$ if $\theta_{1}+\cdots+\theta_{h} \geq 1$.

Since $a_{1}<l^{\prime}$, the assertion (a) is obvious when $h=1$. Now assume that

(a) is true for $h-1$. That is, we assume the following is true.

(b) $Y_{\mathbf{i}}\left(\theta_{1}, \ldots, \theta_{h-1}, 0, \ldots, 0\right) v_{\kappa+\lambda}=0$ if $\theta_{1}+\cdots+\theta_{h-1} \geq 1$.

Set $m_{h+1}:=F_{i_{h+1}}^{\left(a_{h+1}\right)} \cdots F_{i_{r}}^{\left(a_{r}\right)} v_{\kappa+\lambda}$, then we have

(c) $\left[\begin{array}{c}K_{i_{h}}, \theta l^{\prime}-\left(a_{h}-\theta^{\prime} l^{\prime}\right) \\ \theta l^{\prime}\end{array}\right] m_{h+1}=\left(\begin{array}{c}\theta^{\prime}+\theta \\ \theta\end{array}\right) m_{h+1} \quad$ for $\quad \theta, \theta^{\prime} \geq 0$. (Where $\left(\begin{array}{c}\theta^{\prime}+\theta \\ \theta\end{array}\right)$ is the ordinary binomial coefficient.)

Using (c), Lemma 4.4 (iv), Lemma 4.5 and Lemma 4.6, we get

(d) $\quad E_{i_{h}}^{\left(l^{\prime}\right)} Y_{\mathbf{i}}\left(\theta_{1}, \ldots, \theta_{h-1}, 0, \ldots, 0\right) v_{\kappa+\lambda}=Y_{\mathbf{i}}\left(\theta_{1}, \ldots, \theta_{h-1}, 1,0, \ldots, 0\right) v_{\kappa+\lambda}=0$.

Assume that $\theta_{h} \geq 1$ and we have

(e) $Y_{\mathbf{i}}\left(\theta_{1}, \ldots, \theta_{h-1}, \theta_{h}-1,0, \ldots, 0\right) v_{\kappa+\lambda}=0$ if $\theta_{1}+\cdots+\theta_{h-1}+\theta_{h}-1 \geq 1$.

Using (c), (e), Lemma 4.4 (iv), Lemma 4.5 and Lemma 4.6, we get (f) $\quad E_{i_{h}\left(I^{\prime}\right)} Y_{\mathbf{i}}\left(\theta_{1}, \ldots, \theta_{h-1}, \quad \theta_{h}-1, \quad 0, \ldots, 0\right) v_{\kappa+\lambda}=\left(\begin{array}{c}2 \theta_{h}-1 \\ \theta_{h}\end{array}\right) Y_{\mathbf{i}}\left(\theta_{1}, \ldots, \theta_{h-1}, \quad \theta_{h}\right.$,
$0, \ldots, 0) v_{\kappa+\lambda}=0$.

Thus we have proved the assertion (a) by using induction on $h$ as well as on $\theta_{h}$. Take $h=r$, we obtain the lemma.

Lemma 4.8. Let $\mu \in \mathbf{Z}_{+}^{n}$ and $\lambda \in \mathbf{Z}_{+, 1}^{n}$. Then the submodule of $L_{\xi}(\mathbf{l} \mu) \otimes$ $V_{\xi}(\kappa+\lambda)$ generated by $\bar{v}_{\mathbf{1} \mu} \otimes v_{\kappa+\lambda}$ is isomorphic to $V_{\xi}(\mathbf{l} \mu+\kappa+\lambda)$.

Proof. By 3.3 (i), we have a $U_{\xi}$-homomorphism

$$
V_{1}:=V_{\xi}(\mathbf{l} \mu+\kappa+\lambda) \rightarrow V:=L_{\xi}(\mathbf{l} \mu) \otimes V_{\xi}(\kappa+\lambda),
$$

which carries $m_{1}:=v_{\mathbf{1} \mu+\kappa+\lambda}$ to $m:=\bar{v}_{\mathbf{1} \mu} \otimes v_{\kappa+\lambda}$. By $2.2(\mathrm{v}), y_{\lambda}^{\prime} m=\bar{v}_{\mathbf{1} \mu} \otimes y_{\lambda}^{\prime} v_{\kappa+\lambda}$ $\neq 0$. But $y_{\lambda}^{\prime} m_{1}$ generates the unique irreducible submodule of $V_{1}$ (Theorem 
3.7 (ii)). Therefore, the submodule of $V$ generated by $m$ is isomorphic to $V_{1}$. The lemma is proved.

4.9. Proof of Theroem 4.2. (A). We first prove that part (i) implies part (ii). Such an implication will be needed to simplify the induction proof of (i) for type $B_{n}, C_{n}, F_{4}$.

Assume that (i) is true. Since $x_{\lambda}^{\prime} v_{1 \mu+\kappa+\lambda} \neq 0$ and has the same weight with $y_{\lambda}^{\prime} v_{1 \mu+\kappa+\lambda}$, by (i) and Theorem 3.7, we can find a non-zero number $\eta \in \mathbf{Q}(\xi)$ such that $x_{\lambda}^{\prime}-\eta y_{\lambda}^{\prime} \in I_{1 \mu}^{-}+\kappa+\lambda$. Because of $\alpha<6\left(\alpha_{1}+\alpha_{2}+\cdots+\alpha_{n}\right)$ and $l_{\alpha} \leq l$ for every $\alpha \in R^{+}$, we see

(a) $\lambda-w_{0} \lambda \leq \kappa-w_{0} \kappa=2 \kappa=\sum_{\alpha \in R^{+}}\left(l_{\alpha}-1\right) \alpha<6 \operatorname{lr}\left(\alpha_{1}+\alpha_{2}+\cdots+\alpha_{n}\right)$,

where $r=\left|R^{+}\right|$.

Choose $\mu \in \mathbb{Z}_{+}^{n}$ such that $\left\langle\mu, \alpha_{i}^{\vee}\right\rangle>6 l r$ for all $i$. According to the definition of $I_{1 \mu+\kappa+\lambda}^{-}$(see 1.2) we see that $x_{\lambda}^{\prime}-\eta y_{\lambda}^{\prime} \in I_{1 \mu+\kappa+\lambda}^{-}$is equivalent to $x_{\lambda}^{\prime}-\eta y_{\lambda}^{\prime}=0$. So part (i) implies part (ii).

Now we prove (i) case by case. According to Lemma 4.6 and Lemma 4.8 , it suffices to prove the following assertion.

$(*) \quad$ In $L_{\xi}(1 \mu) \otimes V_{\xi}(\kappa+\lambda)$ we have $x_{\lambda}^{\prime}\left(\bar{v}_{\mathbb{1} \mu} \otimes v_{\kappa+\lambda}\right)=\bar{v}_{\mathbb{1} \mu} \otimes x_{\lambda}^{\prime} v_{\kappa+\lambda}$.

We need the following formula [L4, 1.3 (b) p.91].

(†) In $U_{\xi}$ we have $\Delta\left(F_{i}^{(a)}\right)=\sum_{h=0}^{a} \xi^{-d_{i} h(a-h)} F_{i}^{(h)} \otimes K_{i}^{-h} F_{i}^{(a-h)}$ for $i=1,2, \ldots, n$,
$a \geq 0$.

Recall that we have (see $2.2(\mathrm{v})$ )

(†) In $L_{\xi}(\mathbb{R} \mu), F_{\alpha} \bar{v}_{\mathbf{I}}=0$ if $l_{\alpha} \geq 2$.

(B) Using $(\dagger),(\ddagger)$, and Lemma 4.7 we see that $(*)$ is true when the Cartan matrix $\left(a_{i j}\right)$ is symmetric.

(C) Assume that the Cartan matrix $\left(a_{i j}\right)$ is of type $B_{n}$. Keep the notations in (b). of the proof of Lemma 4.6. Given non-negative integers $\theta_{n}, \ldots, \theta_{1}, \theta_{2}^{\prime}, \ldots, \theta_{n}^{\prime}$, set

$Y\left(\theta_{n}, \ldots, \theta_{1}, \theta_{2}^{\prime}, \ldots, \theta_{n}^{\prime}\right):=F_{n}^{\left(a_{n}-\theta_{n} l_{n}\right)} \cdots F_{2}^{\left(a_{2}-\theta_{2} l_{2}\right)} F_{1}^{\left(b_{1}-\theta_{1} l_{1}\right)} F_{2}^{\left(b_{2}-\theta_{2}^{\prime} l_{2}\right)} \cdots F_{n}^{\left(b_{n}-\theta_{n}^{\prime} l_{n}\right)} x_{\lambda, w}^{\prime}$.

Using (b3) and (b4) of the proof of Lemma 4.6, completely as the argument for Lemma 4.7 we get

(C1) $Y\left(\theta_{n}, \ldots, \theta_{1}, \theta_{2}^{\prime}, \ldots, \theta_{n}^{\prime}\right) v_{\kappa+\lambda}=0$ if $\theta_{n}+\cdots+\theta_{1}+\theta_{2}^{\prime}+\cdots+\theta_{n}^{\prime} \geq 1$.

Regard $\left\{\alpha_{1},-\alpha_{1}\right\}$ as a root system of type $B_{1}$, then obviously (i) is true for type $B_{1}$. Assume that (i) is true for type $B_{n-1}$. Then according to (A) we have $x_{\lambda, w}^{\prime} \in \mathrm{dg}_{\xi}^{-}$. Now using $(\dagger),(\ddagger)$, and $(\mathrm{C} 1)$ we see that $(*)$ is true for type $B_{n}$. 
(D) Similarly, we prove $(*)$ for type $C_{n}$.

(E) Assume that the Cartan matrix $\left(a_{i j}\right)$ is of type $F_{4}$. Keep the notations in (d) of the proof of Lemma 4.6. Given non-negatives integers $\theta_{1}, \ldots, \theta_{14}$, set

$$
Y\left(\theta_{1}, \ldots, \theta_{14}\right):=Y_{1}\left(\theta_{1}, \ldots, \theta_{4}\right) Y_{2}\left(\theta_{5}, \ldots, \theta_{8}\right) Y_{3}\left(\theta_{9}, \theta_{10}\right) Y_{4}\left(\theta_{11}, \ldots, \theta_{14}\right) x_{\lambda, w}^{\prime} .
$$

For simplicity, we use the following consequence of Theorem 4.2 for type $B_{3}$ (cf. Corollary 4.10 (ii)).

(E1) $Y_{1}\left(\theta_{1}, \ldots, \theta_{4}\right) F_{2}^{\left(\lambda_{2}\right)} F_{3}^{\left(\lambda_{2}+\lambda_{3}\right)} F_{2}^{\left(\lambda_{2}+2 \lambda_{3}\right)} F_{3}^{\left(\lambda_{3}\right)}=0$ if $\theta_{1}+\cdots+\theta_{4} \geq 1$.

Using (d3-d5) of the proof of Lemma 4.6 as well as (E1), completely as the argument for Lemma 4.7 we get

(E2) $Y\left(\theta_{1}, \ldots, \theta_{14}\right) v_{\kappa+\lambda}=0$ if $\theta_{1}+\cdots+\theta_{14} \geq 1$.

By (D) and (A) we know that $x_{\lambda, w}^{\prime} \in \mathbf{u}_{\xi}^{-}$. Now using (†), (†), and (E2) we see that $(*)$ is true for type $F_{4}$.

The theorem is proved.

Corollary 4.10. Keep the notations in 4.7 and 4.9.

(i) Assume that $\left(a_{i j}\right)$ is symmetric. Then $Y_{\mathbf{i}}\left(\theta_{1}, \ldots, \theta_{r}\right)=0$ if $\theta_{1}+\cdots+\theta_{r} \geq 1$.

(ii) Assume that $\left(a_{i j}\right)$ is of type $B_{n}$. Then $Y\left(\theta_{n}, \ldots, \theta_{1}, \theta_{2}^{\prime}, \ldots, \theta_{n}^{\prime}\right)=0$ if $\theta_{n}+\cdots+\theta_{1}+\theta_{2}^{\prime}+\cdots+\theta_{n}^{\prime} \geq 1$. A similar result holds for type $C_{n}$.

(iii) Assume that $\left(a_{i j}\right)$ is of type $F_{4}$. Then $Y\left(\theta_{1}, \ldots, \theta_{14}\right)=0$ if $\theta_{1}+\cdots+\theta_{14}$ $\geq 1$.

Where $Y_{\mathbf{i}}\left(\theta_{1}, \ldots, \theta_{r}\right), Y\left(\theta_{n}, \ldots, \theta_{1}, \theta_{2}^{\prime}, \ldots, \theta_{n}^{\prime}\right), Y\left(\theta_{1}, \ldots, \theta_{14}\right)$ are elements in $U_{\xi}^{-}$. Recall that $F_{i}^{(a)}=0$ for all $i$ and $a<0$, see 1.1 .

Proof. We give a proof of (i). The proofs of (ii) and (iii) are similar. Using Lemma $4.7,(\dagger)$ and $(\$)$ in 4.9 we see that

$$
Y_{\mathbf{i}}\left(\theta_{1}, \ldots, \theta_{r}\right)\left(\bar{v}_{\mathbf{1} \mu} \otimes v_{\kappa+\lambda}\right)=0, \quad \text { if } \theta_{1}+\cdots+\theta_{r} \geq 1 .
$$

Using Lemma 4.8 and an argument as in 4.9 (A) we know that (i) is true.

4.11. By Lemma 4.4 (iii), Theorem 4.2 is actually equivalent to the assertion $x_{\lambda}^{\prime} \in \mathbf{u}_{\xi}^{-}$when $\lambda$ is I-restricted. For type $B_{2}$, using the commutation relation in $\left[\mathrm{L} 4,5.3\right.$ (i), p.96] we see easily that if $\lambda$ is l-restricted then $x_{\lambda}^{\prime} \in \mathbf{u}_{\xi}^{-}$. For type $A_{n}$ there is a naive argument for the fact, which is based on the following Lemma 4.12. We need a notation. Given $i \in[1, n]$, let $\mathscr{H}_{i}$ be the $\mathbf{Q}(\xi)$-subspace of $U_{\xi}^{-}$spanned by the elements $F_{\beta_{1}}^{\left(a_{1}\right)} F_{\beta_{2}}^{\left(a_{2}\right)} \ldots F_{\beta_{r}}^{\left(a_{r}\right)}$ for $a_{1}, \ldots, a_{r} \in \mathbf{N}$ satisfying $a_{h} \leq l_{\beta_{h}}-1$ whenever $\beta_{h}-\alpha_{i} \in \mathbf{N} R^{+}(h=1, \ldots, r)$. Obviously, $\bigcap_{i=1}^{n}$ $\mathscr{H}_{i}=\mathbf{u}_{\xi}^{-}$.

Lemma 4.12. Let $x$ be an element in $U_{\xi}$. Assume that $x$ is expressed as a $\mathbf{Q}(\xi)$-linear combination of some monomials $z_{1}, \ldots, z_{h}$ of $F_{\alpha}^{(a)}\left(\alpha \in R^{+}, a \in \mathbf{N}\right)$. 
Given $i \in[1, n]$. If $a \leq l_{\alpha}-1$ whenever $F_{\alpha}^{(a)}$ appears in some monomial $z_{k}$ and $\alpha-\alpha_{i} \in \mathbf{N} R^{+}$, then $x \in \mathscr{H}_{i}$.

Proof. Using commutation relations in [L4, 5.3-5.4, pp.95-97] and [L4, Theorem 6.6, pp. 103-104].

4.13. Now we give a simple proof of Theorem 4.2 for type $A_{n}$ by using Lemma 4.12. By Lemma 4.4 (ii), it suffices to prove that $x_{\lambda}^{\prime} \in \mathbf{u}_{\xi}^{-}$when $\lambda$ is l-restricted. We use induction on $n$. Set $\lambda_{i}:=\left\langle\lambda, \alpha_{i}^{\vee}\right\rangle$ for $i=1,2, \ldots, n$. When $1 \leq i \leq j \leq n$ we also write $\lambda_{i, j}$ for $\lambda_{i}+\lambda_{i+1}+\cdots+\lambda_{j}$. Then

$$
x_{\lambda}^{\prime}=F_{1}^{\left(\lambda_{n}\right)} F_{2}^{\left(\lambda_{n-1, n}\right)} \cdots F_{n}^{\left(\lambda_{1, n}\right)} F_{1}^{\left(\lambda_{n-1}\right)} F_{2}^{\left(\lambda_{n-2, n-1}\right)} \cdots F_{n-1}^{\left(\lambda_{1}, n-1\right)} \cdots F_{1}^{\left(\lambda_{2}\right)} F_{2}^{\left(\lambda_{1,2}\right)} F_{1}^{\left(\lambda_{1}\right)} .
$$

Note that $l_{1}=\cdots=l_{n}$, by Lemma 4.12 we get (a) $x_{\lambda}^{\prime} \in \mathscr{H}_{1}$. Symmetrically, we have $x_{\lambda}^{\prime} \in \mathscr{H}_{n}$.

Let $w=s_{1} s_{2} s_{1} s_{3} s_{2} s_{1} \cdots s_{n-1} \cdots s_{2} s_{1}$ (the longest element of the group generated by $\left.s_{1}, \ldots, s_{n-1}\right)$. Set

$$
\begin{gathered}
y:=F_{1}^{\left(\lambda_{n-1}\right)} F_{2}^{\left(\lambda_{n-2, n-1}\right)} F_{1}^{\left(\lambda_{n-2}\right)} F_{3}^{\left(\lambda_{n-3, n-1}\right)} F_{2}^{\left(\lambda_{n-3, n-2}\right)} F_{1}^{\left(\lambda_{n-3}\right)} \cdots F_{n-2}^{\left(\lambda_{2}, n-1\right)} \cdots F_{1}^{\left(\lambda_{2}\right)}, \\
y^{\prime}:=F_{n-1}^{\left(\lambda_{1, n-1}\right)} \cdots F_{2}^{\left(\lambda_{1,2}\right)} F_{1}^{\left(\lambda_{1}\right)} .
\end{gathered}
$$

Then $x_{\lambda, w}^{\prime}=y y^{\prime}$. By induction hypothesis, $y, x_{\lambda, w}^{\prime} \in \mathbf{u}_{\xi}^{-}$. By 2.2 (ii), then $x_{\lambda, w}^{\prime}=y z$ for some $z \in \mathbf{u}_{\xi}^{-}$. Note that

$$
x_{\lambda}^{\prime}=F_{1}^{\left(\lambda_{n}\right)} F_{2}^{\left(\lambda_{n-1, n}\right)} \cdots F_{n-1}^{\left(\lambda_{2, n}\right)} y F_{n}^{\left(\lambda_{1, n}\right)} z \quad \text { and } \quad F_{1}^{\left(\lambda_{n}\right)} F_{2}^{\left(\lambda_{n-1, n}\right)} \cdots F_{n-1}^{\left(\lambda_{2, n}\right)} y=x_{v, w}^{\prime},
$$

where $v:=\left(\lambda_{2}, \ldots, \lambda_{n}, \lambda_{1}\right)$. According to induction hypothesis, $x_{v, w}^{\prime} \in \mathbf{u}_{\xi}^{-}$. Now by Lemma 4.12, $x_{\lambda}^{\prime}=x_{v, w}^{\prime} F_{n}^{\left(\lambda_{1, n}\right)} z \in \bigcap_{i=1}^{n-1} \mathscr{H}_{i}$. Combine this and (a) we see $x_{\lambda}^{\prime} \in \bigcap_{i=1}^{n} \mathscr{H}_{i}=\mathbf{u}_{\xi}^{-}$.

\section{§5. Main Results}

5. 1. In this section we give the main results of the paper. Essentially, they re-express some results in previous sections. Recall that in 1.4 we have defined the element $x_{\lambda} \in U_{\xi}^{-}$for every $\lambda$ in $\mathbb{Z}_{+}^{n}$.

Theorem 5.2. Assume that $U_{\xi}$ has no factors of type $G_{2}$. If $\lambda$ is I-restricted, then $x_{\lambda}$ and $x_{\lambda}^{\prime}$ are elements in $\mathbf{u}_{\xi}^{-}$.

Proof. By Theorem 4.2 (ii), $x_{\lambda}^{\prime}$ is an element in $\mathbf{u}_{\xi}^{-}$. We have $x_{\lambda}=x_{-w_{0} \lambda}^{\prime}$. Note that $-w_{0} \lambda$ is also $\mathbb{l}$-restricted, by Theorem 4.2 (ii), $x_{\lambda} \in \mathbb{Q}_{\xi}^{-}$.

Theorem 5.3. Assume that $U_{\xi}$ has no factors of type $G_{2}$. Let $\lambda=$ $\left(\lambda_{1}, \lambda_{2}, \ldots, \lambda_{n}\right) \in \mathbb{Z}_{+}^{n}$ be l-restricted and let $\sigma=\left(\sigma_{1}, \sigma_{2}, \ldots, \sigma_{n}\right) \in\{ \pm 1\}^{n}$. Denote by $\mathbf{u}_{\xi}(\lambda, \sigma)$ the left ideal of $\mathbb{u}_{\xi}$ generated by the elements $E_{\alpha}, K_{i}-\sigma_{i} \xi^{\lambda_{1} d_{1}}$ $\left(\alpha \in R_{l}^{+}, i=1,2, \ldots, n\right)$ and elements $F \in \mathbf{u}_{\xi}^{-}$such that $F x_{\kappa-\lambda}=0$. Then 
(i) $\mathbf{u}_{\xi} / \mathbf{u}_{\xi}(\lambda, \sigma)$ is an irreducible $\mathbf{u}_{\xi}$-module. Moreover, as $\mathbf{u}_{\xi}$-modules, $L_{\xi}(\lambda, \sigma)$ is isomorphic to $\mathbf{u}_{\xi} / \mathbf{u}_{\xi}(\lambda, \sigma)$.

(ii) For any $\gamma \in \mathbf{N} R^{+}$, denote $\mathbf{u}_{\xi, \gamma}^{-}$the set of all elements in $\mathbf{u}_{\xi}^{-}$of degree $\gamma$, and set $\mathbf{n}_{\xi}(\lambda, \gamma):=\left\{F \in \mathbf{u}_{\xi, \gamma}^{-} \mid F x_{\kappa-\lambda}=0\right\}$. Then

$$
\operatorname{dim}_{\mathbf{Q}(\xi)} L_{\xi}(\lambda, \sigma)_{\lambda-\gamma, \sigma}=\operatorname{dim}_{\mathbf{Q}(\xi)} \mathbf{u}_{\xi, \gamma}^{-}-\operatorname{dim}_{\mathbf{Q}(\xi)} \mathbf{n}_{\xi}(\lambda, \gamma) .
$$

In particular, we have

$$
\operatorname{dim}_{\mathbf{Q}(\xi)} L_{\xi}(\lambda, \sigma)=\prod_{\alpha \in R^{+}} l_{\alpha}-\operatorname{dim}_{\mathbf{Q}(\xi)}\left\{F \in \mathbf{u}_{\xi}^{-} \mid F x_{\kappa-\lambda}=0\right\} .
$$

Proof. (i) Let $\tilde{J}_{\lambda, \sigma}$ be the left ideal of $\tilde{\mathbf{u}}_{\xi}$ generated by the elements $E_{\alpha}, K_{i}-\sigma_{i} \xi^{\lambda_{1} d_{2}},\left[\begin{array}{c}K_{i}, c \\ a\end{array}\right]-\sigma_{i}^{a}\left[\begin{array}{c}\lambda_{i}+c \\ a\end{array}\right]_{d_{l}, \xi}\left(\alpha \in R_{l}^{+}, i=1,2, \ldots, n, c \in \mathbf{Z}, a \in \mathbf{N}\right)$ and elements $F \in \mathbf{u}_{\xi}^{-}$such that $F x_{\kappa-\lambda}=0$. Since $x_{\kappa-\lambda}=x_{\kappa+w_{0} \lambda}^{\prime}$, by Theorem 4.2 (ii), Corollary 2.7 (ii) and Prop. 2.6 (ii) we see that $\tilde{\mathbf{u}}_{\xi} / \tilde{J}_{\lambda, \sigma} \simeq \widetilde{L}_{\xi}(\lambda, \sigma)$. But $\lambda$ is I-restricted, so the restriction to $\mathbf{u}_{\xi}$ of $\tilde{L}_{\xi}(\lambda, \sigma)$ is an irreducible $\mathbf{u}_{\xi}$-module. Obviously, the restriction is isomorphic to $\mathbf{u}_{\xi} / \mathbf{u}_{\xi}(\lambda, \sigma)$. Since $\tilde{L}_{\xi}(\lambda, \sigma)$ is the restriction to $\tilde{\mathbf{u}}_{\xi}$ of the irreducible $U_{\xi}$-module $L_{\xi}(\lambda, \sigma)$, so as $\mathbf{u}_{\xi}$-modules, $L_{\xi}(\lambda, \sigma)$ is isomorphic to $\mathbf{u}_{\xi} / \mathbf{u}_{\xi}(\lambda, \sigma)$.

Part (ii) is an immediate consequence of part (i).

The theorem is proved.

Theorem 5.4. Assume that $U_{\xi}$ has no factors of type $G_{2}$. Let $\lambda, \mu \in \mathbf{Z}_{+}^{n}$ and let $\sigma=\left(\sigma_{1}, \sigma_{2}, \ldots, \sigma_{n}\right) \in\{ \pm 1\}^{n}$. Assume that $\lambda$ is l-restricted. Denote by $J_{1 \mu+\lambda, \sigma}$ the left ideal of $U_{\xi}$ generated by all elements in $I_{1 \mu+\lambda, \sigma}$ (see 1.2 for the definition) and elements $F \in \mathbf{u}_{\xi}^{-}$such that $F x_{\kappa-\lambda}=0$. Then $U_{\xi} / J_{1 \mu+\lambda, \sigma} \simeq$ $L_{\xi}(\mathbf{l} \mu+\lambda, \sigma)$.

Proof. Since $L_{\xi}(\mathbf{l} \mu+\lambda, \sigma)$ is a quotient module of $V_{\xi}(1 \mu+\lambda, \sigma)$, we have $I_{\mathbf{1} \mu+\lambda, \sigma} \bar{v}_{\mathbf{l} \mu+\lambda, \sigma}=0$. Noting that $\left.L_{\xi}(\mathbf{l} \mu+\lambda, \sigma) \simeq L_{\xi}(\mathbf{l} \mu, \mathbf{1}) \otimes L_{\xi}(\lambda, \sigma)\right)$ (see 2.2 (iv)), by 2.2 (v) and Theorem 5.3 (i) we know that $F \bar{v}_{1 \mu+\lambda, \sigma}=0$ if $F \in \mathbf{u}_{\xi}^{-}$and $F x_{\kappa-\lambda}=0$. Therefore we have $J_{1 \mu+\lambda, \sigma} \bar{v}_{1 \mu+\lambda, \sigma}=0$. Note that

(a) $L_{\xi}(\mathbf{l} \mu, \mathbf{1}) \otimes L_{\xi}(\kappa, \sigma) \simeq L_{\xi}(\mathbf{l} \mu, \mathbf{1}) \otimes V_{\xi}(\kappa, \sigma) \simeq V_{\xi}(\mathbf{l} \mu+\kappa, \sigma)$.

Let $z_{1}, z_{2}, \ldots, z_{k}, \ldots$, be a $\mathbf{Q}(\xi)$-basis of $U_{\xi, l}^{-}$such that

(b) The elements $z_{b} \bar{v}_{\mathbf{l} \mu}(b=1,2, \ldots, k)$ form a $\mathbf{Q}(\xi)$-basis of the irreducible module $L_{\xi}(\mathbf{l} \mu)$, and $z_{b} \bar{v}_{\mathbf{l} \mu}=0$ for $b=k+1, k+2 \ldots$.

Let $\bar{I}$ be the $\mathbf{Q}(\xi)$-space spanned by the elements $z_{h} F\left(1 \leq h \leq k, F \in \mathbf{u}_{\xi}^{-}\right)$. According to (a) and (b) we have $\bar{I}+I_{1 \mu+\kappa, \sigma}^{-}=U_{\xi}^{-}$. Since $I_{1 \mu+\kappa, \sigma}^{-} \subseteq I_{1 \mu+\lambda, \sigma}$, as $\mathbf{Q}(\xi)$-spaces we have

$$
U_{\xi} / J_{\mathbf{1} \mu+\lambda, \sigma} \simeq U_{\xi}^{-} / U_{\xi}^{-} \cap J_{\mathbf{1} \mu+\lambda, \sigma} \simeq \bar{I} / \bar{I} \cap J_{\mathbf{1} \mu+\lambda, \sigma} .
$$

By Theorem 5.3, $\operatorname{dim}_{\mathbf{Q}(\xi)} \bar{I} \cap J_{\mathbf{1} \mu+\lambda, \sigma} \geq k\left(\operatorname{dim}_{\mathbf{Q}(\xi)} \mathbf{u}_{\xi}^{-}-\operatorname{dim}_{\mathbf{Q}(\xi)} L_{\xi}(\lambda, \sigma)\right)$. Since 
$\operatorname{dim}_{\mathbf{Q}(\xi)} \bar{I}=k \operatorname{dim}_{\mathbf{Q}(\xi)} \mathbf{u}_{\xi}^{-}$, we have

$$
\operatorname{dim}_{\mathbf{Q}(\xi)} U_{\xi} / J_{\mathbf{1} \mu+\lambda, \sigma} \leq k \operatorname{dim}_{\mathbf{Q}(\xi)} L_{\xi}(\lambda, \sigma)=\operatorname{dim}_{\mathbf{Q}(\xi)} L_{\xi}(\mathbb{l} \mu+\lambda, \sigma) .
$$

This forces that $U_{\xi} / J_{1 \mu+\lambda, \sigma}$ and $L_{\xi}(1 \mu+\lambda, \sigma)$ have the same dimension and as $U_{\xi}$-modules, they are isomorphic.

The theorem is proved

From the above proof we get the following result.

Corollary 5.5. Keep the setup in Theorem 5.4. Then the left ideal $J_{\mathbf{1} \mu+\lambda, \sigma} \cap U_{\xi}^{-}$of $U_{\xi}^{-}$is generated by the elements $F_{i}^{\left(l_{2} \mu_{1}+l_{\mathbf{l}}\right)}(i=1,2, \ldots, n)$ and elements $F \in \mathbf{u}_{\xi}^{-}$such that $F x_{\kappa-\lambda}=0$.

\section{§6. Hyperalgebra}

6.1. In this section we consider the hyperalgebra $\mathfrak{U}_{\mathfrak{f}}$ along the same line in the previous sections, the discussion will be brief. We often omit those proofs which are essentially the same as in the previous sections.

Recall that $\mathfrak{g}$ is a semisimple Lie algebra over $\mathbf{C}$ and $\mathfrak{U}$ is the universal enveloping algebra of $g$. Let $e_{\alpha}, f_{\alpha}, h_{i}\left(\alpha \in R^{+}, i=1,2, \ldots, n\right)$ be a Chevalley basis of g. We also write $e_{i}, f_{i}$ for $e_{\alpha_{1}}, f_{\alpha_{i}}(i=1,2, \ldots, n)$. The Kostant $\mathbb{Z}$-form $\mathfrak{U}_{\mathbf{Z}}$ of $\mathfrak{U}$ is the $\mathbb{Z}$-subalgebra of $\mathfrak{U}$ generated by the elements $e_{\alpha}^{(k)}:=e_{\alpha}^{k} / k !, f_{\alpha}^{(k)}:=$ $f_{\alpha}^{k} / k$ ! for $\alpha \in R^{+}$and $k \in \mathbb{N}$. Set

$$
\left(\begin{array}{c}
h_{i}+c \\
k
\end{array}\right):=\frac{\left(h_{i}+c\right)\left(h_{i}+c-1\right) \cdots\left(h_{i}+c-k+1\right)}{k !},
$$

then $\left(\begin{array}{c}h_{\mathbf{z}}+c \\ k\end{array}\right) \in \mathfrak{U}_{\mathbf{Z}}$, for $i=1,2, \ldots, n, c \in \mathbb{Z}, k \in \mathbf{N}$. Let $\mathfrak{f}$ be an algebraically closed field of prime characteristic $p$. Define $\mathfrak{U}_{\mathfrak{f}}:=\mathfrak{U}_{\mathbb{Z}} \otimes \mathfrak{f}$ and call $\mathfrak{U}_{\mathfrak{f}}$ the hyperalgebra associated to $\mathfrak{g}$ and $\mathfrak{f}$. Let $\mathfrak{U}_{\mathfrak{f}}^{+}, \mathfrak{U}_{\mathfrak{f}}^{-}, \mathfrak{U}_{\mathfrak{f}}^{0}$ be the positive part, negative part, zero part of $\mathfrak{U}_{\mathfrak{i}}$ respectively. To simplify notation, the images in $\mathfrak{U}_{\mathfrak{f}}$ of $e_{\alpha}^{(k)}, f_{\alpha}^{(k)},\left(\begin{array}{c}h_{i}+c \\ k\end{array}\right)$, etc. will be denoted by the same notations respectively. The algebra $\mathfrak{U}_{\mathfrak{t}}$ is a Hopf algebra, the coproduct, denoted also by $\Delta$, is defined as follows:

$$
\Delta\left(e_{\alpha}^{(k)}\right)=\sum_{q=0}^{k} e_{\alpha}^{(q)} \otimes e_{\alpha}^{(k-q)}, \quad \Delta\left(f_{\alpha}^{(k)}\right)=\sum_{q=0}^{k} f_{\alpha}^{(q)} \otimes f_{\alpha}^{(k-q)} .
$$

The tensor product of two $\mathfrak{U}_{\mathfrak{f}}$-modules then has a natural $\mathfrak{U}_{\mathfrak{f}}$-module structure by means of the coproduct, and the antipode can be used to define the dual module of a $\mathfrak{U}_{\mathfrak{t}}$-module.

Given a positive integer $a$, let $\mathfrak{u}_{a}$ be the $a$-th Frobenius kernel of $\mathfrak{U}_{\mathfrak{f}}$. By definition, $\mathfrak{u}_{a}$ is the subalgebra of $\mathfrak{U}_{\mathfrak{f}}$ generated by the elements $e_{\alpha}^{(k)}, f_{\alpha}^{(k)},\left(\begin{array}{c}h_{k} \\ k\end{array}\right)$ for $\alpha \in R^{+}, i=1,2, \ldots, n, 0 \leq k<p^{a}$. Denote by $\mathfrak{u}_{a}^{+}, \mathfrak{u}_{a}^{-}, \mathfrak{u}_{a}^{0}$ the positive part, 
negative part, zero part of $\mathfrak{u}_{a}$ respectively. Let $\tilde{\mathfrak{u}}_{a}$ be the subalgebra of $\mathfrak{U}_{\mathfrak{f}}$ generated by all elements in $\mathfrak{u}_{a} \cup \mathfrak{U}_{\mathfrak{f}}^{0}$, then $\tilde{\mathfrak{u}}_{a}=\mathfrak{u}_{a}^{-} \mathfrak{U}_{\mathfrak{f}}^{0} \mathfrak{u}_{a}^{+}$. Let $\mathfrak{U}_{\mathfrak{f}, a}$ be the subalgebra of $\mathfrak{U}_{\mathfrak{f}}$ generated by the elements $1, e_{\alpha}^{\left(p^{b}\right)}, f_{\alpha}^{\left(p^{b}\right)},\left(\begin{array}{c}h_{\mathfrak{l}} \\ p^{b}\end{array}\right)$ for $\alpha \in R^{+}$, $i=1,2, \ldots, n, b \geq a$. Let $\mathfrak{U}_{\mathfrak{f}, a}^{+}, \mathfrak{U}_{\mathfrak{f}, a}^{-}, \mathfrak{U}_{\mathfrak{t}, a}^{0}$ be the positive part, negative part, zero part of $\mathfrak{U}_{\mathfrak{t}, a}$ respectively. The following results are easy to check.

(i) Let $g \in \mathfrak{u}_{a}$. We have $e_{i}^{\left(p^{a}\right)} g-g e_{i}^{\left(p^{a}\right)} \in \mathfrak{u}_{a}$ and $f_{i}^{\left(p^{a}\right)} g-g f_{i}^{\left(p^{a}\right)} \in \mathfrak{u}_{a}$.

(ii) Let $\left\{g_{k}\right\}$ be a basis of $\mathfrak{u}_{a}^{-}$and $\left\{G_{q}\right\}$ be a basis of $\mathfrak{U}_{\mathfrak{i}, a}^{-}$, then $\left\{g_{k} G_{q}\right\}$ is a basis of $\mathfrak{U}_{\mathfrak{i}}^{-}$, so is $\left\{G_{q} g_{k}\right\}$.

(iii) There exists a unique surjective $\mathfrak{f}$-algebra homomorphism $\mathfrak{U}_{\mathfrak{f}} \rightarrow \mathfrak{U}_{\mathfrak{f}}$ such that $e_{\alpha}^{\left(k p^{a}\right)} \rightarrow e_{\alpha}^{(k)}, f_{\alpha}^{\left(k p^{a}\right)} \rightarrow f_{\alpha}^{(k)},\left(\begin{array}{c}h_{i} \\ k p^{a}\end{array}\right) \rightarrow\left(\begin{array}{c}h_{i} \\ k\end{array}\right)$ for $\alpha \in R^{+}, i=1,2, \ldots, n, k \in \mathbf{N}$, and such that $e_{\alpha}^{(k)} \rightarrow 0, f_{\alpha}^{(k)} \rightarrow 0,\left(\begin{array}{c}h_{1} \\ k\end{array}\right) \rightarrow 0$ if $k$ is not divisible by $p^{a}$. In particular $\mathfrak{U}_{\mathfrak{f}, a}$ is isomorphic to $\mathfrak{U}_{\mathfrak{f}}$.

Proof. The $\mathrm{f}$-algebra homomorphism is obtained from the $a$-th Frobenius map of the simply connected, semisimple algebraic group (associated to $\mathfrak{g}$ ) over $\mathfrak{f}$. One also can see (iii) by using the commutation relations among the generators of $\mathfrak{U}_{\mathfrak{f}}$.

We order $R^{+}$so that $R^{+}=\left\{\beta_{1}, \beta_{2}, \ldots, \beta_{r}\right\}$ where $\beta_{i} \leq \beta_{j}$ implies that $i \geq j$. For $a \in \mathbb{N}$, set $f_{\left(p^{a}-1\right) \rho}=f_{\beta_{1}}^{\left(p^{a}-1\right)} f_{\beta_{2}}^{\left(p^{a}-1\right)} \cdots f_{\beta_{r}}^{\left(p^{a}-1\right)}$. For $b \geq a$ we set $f_{p^{a}\left(p^{b-a}-1\right) \rho}^{\prime}=f_{\beta_{r}}^{\left(p^{b}-p^{a}\right)} f_{\beta_{r}-1}^{\left(p^{b}-p^{a}\right)} \cdots f_{\beta_{1}}^{\left(p^{b}-p^{a}\right)}$. Since in $\mathfrak{f}$ we have $\left(\begin{array}{c}p^{b}-1 \\ p^{a}-1\end{array}\right)=1$ and $f_{p^{a}\left(p^{b-a}-1\right) \rho}^{\prime} f_{\left(p^{a}-1\right) \rho} \in \mathfrak{u}_{b}^{-}$, we get

(iv) Let $a, b \in \mathbf{N}$ with $b \geq a$. Then

$$
f_{\left(p^{b}-1\right) \rho}=f_{p^{a}\left(p^{b-a}-1\right) \rho}^{\prime} f_{\left(p^{a}-1\right) \rho} .
$$

Using commutation relations among $f_{\alpha}^{(k)}\left(\alpha \in R^{+}, k \in \mathbf{N}\right)$ and using induction on $j \in[1, r]$ we get

(v) Let $a, k \in \mathbb{N}$ with $k \geq p^{a}$ and let $j \in[1, r]$. Then for each $i$ in $[1, n]$ we have

$$
f_{i}^{(k)} f_{\beta_{1}}^{\left(p^{a}-1\right)} \cdots f_{\beta_{J}}^{\left(p^{a}-1\right)}=f_{\beta_{1}}^{\left(p^{a}-1\right)} \cdots f_{\beta_{j}}^{\left(p^{a}-1\right)} f_{i}^{(k)}
$$

In particular,

$$
f_{i}^{(k)} f_{\left(p^{a}-1\right) \rho}=f_{\left(p^{a}-1\right) \rho} f_{i}^{(k)} .
$$

6.2. Let $\mathfrak{U}_{\mathfrak{i}}^{\prime}$ be a subalgebra of $\mathfrak{U}_{\mathfrak{f}}$ containing $\mathfrak{U}_{\mathfrak{i}}^{0}$ and let $M$ be a $\mathfrak{U}_{\mathfrak{f}}^{\prime}$-module. Let $\lambda=\left(\lambda_{1}, \lambda_{2}, \ldots, \lambda_{n}\right) \in \mathbb{Z}^{n}$. An element $m \in M$ is called to have weight $\lambda$ if $\left(\begin{array}{c}h_{l} \\ k\end{array}\right) m=\left(\begin{array}{c}\lambda_{1} \\ k\end{array}\right) m$ for $i=1,2, \ldots, n, k \in \mathbb{N}$. Denote by $M_{\lambda}$ the set of all elements in $M$ of weight $\lambda$. We call $\lambda$ a weight of $M$ if $M_{\lambda}$ is not zero. An element

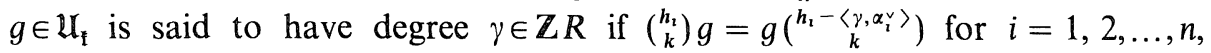
$k \geq 0$. If an element $g$ in $\mathfrak{U}_{\mathfrak{i}}^{\prime}$ has degree $\gamma$, then obviously $g M_{\lambda} \subseteq M_{\lambda-\gamma}$. We list some well known properties and supply proofs for a few of them. The letters $a, b$ will stand for positive integers. 
(i) If $M$ is a finite dimensional $\mathfrak{U}_{\mathfrak{f}}$-module, then $\operatorname{dim} M_{\lambda}=\operatorname{dim} M_{w \lambda}$ for all $\lambda \in \mathbb{Z}^{n}, w \in W$.

Given $\lambda=\left(\lambda_{1}, \lambda_{2}, \ldots, \lambda_{n}\right) \in \mathbb{Z}_{+}^{n}$, let $\mathfrak{I}_{\lambda}^{+}$be the left ideal of $\mathfrak{U}_{\mathfrak{f}}$ generated by the elements $e_{i}^{(k)},\left(\begin{array}{c}h_{1} \\ k\end{array}\right)-\left(\begin{array}{c}\lambda_{1} \\ k\end{array}\right)$ for $i=1,2, \ldots, n, k \geq 1$, and let $\mathfrak{J}_{\lambda}^{-}$be the left ideal of $\mathfrak{U}_{\mathfrak{f}}^{-}$generated by the elements $f_{i}^{\left(k_{i}\right)}$ for $i=1,2, \ldots, n, k_{i} \geq \lambda_{i}+1$. Then let $\mathfrak{I}_{\lambda}$ be the left ideal of $\mathfrak{U}_{\mathfrak{i}}$ generated by all elements in $\mathfrak{I}_{\lambda}^{+} \cup \mathfrak{I}_{\lambda}^{-}$. Then

(ii) The $\mathfrak{U}_{\mathfrak{f}}$-module $V_{\mathfrak{f}}(\lambda):=\mathfrak{U}_{\mathfrak{f}} / \mathfrak{I}_{\lambda}$ is of finite dimension and has a unique irreducible quotient module, denoted by $L_{\mathfrak{i}}(\lambda)$. The dimension of $V_{\mathfrak{i}}(\lambda)$ is given by Weyl's character formula. By abuse of notations, we also denote $v_{\lambda}$ the image in $V_{\mathfrak{f}}(\lambda)$ of the neutral element $1 \in \mathfrak{U}_{\mathfrak{f}}$, and denote $\bar{v}_{\lambda}$ the image in $L_{\mathfrak{f}}(\lambda)$ of $v_{\lambda}$. The map $\lambda \rightarrow L_{\mathfrak{k}}(\lambda)$ defines a bijection between the set $\mathbb{Z}_{+}^{n}$ and the set of isomorphism classes of irreducible $\mathfrak{U}_{\mathfrak{f}}$-modules of finite dimensions.

(iii) When $\lambda$ is $p^{a}$-restricted (i.e. $0 \leq\left\langle\lambda, \alpha_{i}^{v}\right\rangle\left\langle p^{a}\right.$ for $i=1,2, \ldots, n$ ), the restriction to $\mathfrak{u}_{a}$ (resp. $\tilde{\mathfrak{u}}_{a}$ ) of $L_{\mathfrak{f}}(\lambda)$ is an irreducible $\mathfrak{u}_{a}$-module (resp. $\tilde{\mathfrak{u}}_{a}$-module), denote the restriction by $L_{\mathfrak{f}, a}(\lambda)$ (resp. $\tilde{L}_{\mathfrak{f}, a}(\lambda)$ ).

(iv) Assume $\lambda \in p^{a} \mathbb{Z}_{+}^{n}$, then $e_{\alpha}^{(k)} m=f_{\alpha}^{(k)} m=0$ for all $m \in L_{\mathfrak{t}}(\lambda), \alpha \in R^{+}, 1 \leq k<p^{a}$.

Proof. Use 6.1 (iii) and 6.2 (ii).

(v) Assume that $\delta_{0}, \delta_{1}, \ldots, \delta_{b} \in \mathbb{Z}_{+}^{n}$ are $p$-restricted and set $\lambda:=p^{b} \delta_{b}+\cdots+$ $p \delta_{1}+\delta_{0}$. Then (Steinberg's tensor product theorem)

$$
L_{\mathfrak{f}}(\lambda) \simeq L_{\mathfrak{t}}\left(p^{b} \delta_{b}\right) \otimes \cdots \otimes L_{\mathfrak{t}}\left(p \delta_{1}\right) \otimes L_{\mathfrak{t}}\left(\delta_{0}\right) .
$$

Proof. Use (iv) and the trick in the proof of [L2, Theorem 7.4, p.73].

Let $M$ be a $\mathfrak{U}_{\mathfrak{i}}$-module (resp. $\tilde{\mathfrak{u}}_{a}$-module). A nonzero element $m$ in $M$ is called primitive if $m \in M_{\lambda}$ for some $\lambda \in \mathbb{Z}^{n}$ and $e_{i}^{(k)} m=0$ for $i=1,2, \ldots, n$, $k \geq 1$ (resp. $e_{\alpha}^{(k)} m=0$ for all $\alpha \in R^{+}, 1 \leq k \leq p^{a}-1$ ).

(vi) Let $M$ be a finite dimensional $\mathfrak{U}_{t}$-module. Assume that $m \in M$ is a primitive element of weight $\lambda$. Then $\lambda \in \mathbb{Z}_{+}^{n}$ and there is a unique $\mathfrak{U}_{\mathfrak{f}}$-module homomorphism $V_{\mathrm{f}}(\lambda) \rightarrow M$ which carries $v_{\lambda}$ to $m$.

Proof. By (i) we see $s_{i} \lambda \leq \lambda$ for $i=1,2, \ldots, n$, that is $\lambda \in \mathbb{Z}_{+}^{n}$. Assume that $f_{i}^{(k)} m$ is not zero, again by (i) we see $s_{i}\left(\lambda-k \alpha_{i}\right) \leq \lambda$, i.e., $k \leq\left\langle\lambda, \alpha_{i}^{v}\right\rangle$. According to the definition of $V_{\mathfrak{t}}(\lambda)$ we know that the required $\mathfrak{U}_{\mathfrak{t}^{-}}$ homomorphism exists.

Given $\mu=\left(\mu_{1}, \mu_{2}, \ldots, \mu_{n}\right) \in \mathbb{Z}^{n}$, let $\tilde{\mathfrak{J}}_{\mu, a}^{+}$be the left ideal of $\tilde{\mathfrak{u}}_{a}$ generated by the elements $e_{\alpha}^{(k)},\left(\begin{array}{c}h_{t} \\ k^{\prime}\end{array}\right)-\left(\begin{array}{c}\lambda_{t} \\ k^{\prime}\end{array}\right)$ for $\alpha \in R^{+}, i=1,2, \ldots, n, 1 \leq k<p^{a}, k^{\prime} \in \mathbf{N}$. Denote by $\tilde{Z}_{\mathfrak{f}, a}(\mu)$ the Verma module $\tilde{\mathfrak{u}}_{a} / \tilde{\mathfrak{I}}_{\mu, a}^{+}$of $\tilde{\mathfrak{u}}_{a}$ with highest weight $\mu$. We shall denote $\tilde{1}_{\mu, a}$ the image in $\tilde{Z}_{\mathfrak{t}, a}(\mu)$ of the neutral element $1 \in \tilde{\mathfrak{u}}_{a}$. We have (vii) Each Verma module of $\tilde{\mathfrak{u}}_{a}$ has a unique irreducible submodule. Assume that $\lambda$ is $p^{a}$-restricted. Then the irreducible $\tilde{\mathfrak{u}}_{a}$-submodule of $\tilde{Z}_{\mathfrak{i}, a}\left(\left(p^{a}-1\right) \rho+\lambda\right)$ (resp. $\left.\tilde{Z}_{\mathfrak{f}, a}\left(2\left(p^{a}-1\right) \rho+w_{0} \lambda\right)\right)$ is isomorphic to $\tilde{L}_{\mathfrak{f}, a}\left(\left(p^{a}-1\right) \rho+w_{0} \lambda\right)$ (resp. $\left.\tilde{L}_{\mathrm{f}, a}(\lambda)\right)$ and is generated by $f_{\left(p^{a}-1\right) \rho} \tilde{1}_{\left(p^{a}-1\right) \rho+\lambda, a}\left(\operatorname{resp} . f_{\left(p^{a}-1\right) \rho} \tilde{1}_{2\left(p^{a}-1\right) \rho+w_{0} \lambda, a}\right)$, where $\rho=(1, \ldots, 1) \in \mathbb{Z}_{+}^{n}$. In particular, $\tilde{Z}_{\mathfrak{t}, a}\left(\left(p^{a}-1\right) \rho\right)$ is isomorphic to 
$\tilde{L}_{\mathrm{f}, a}\left(\left(p^{q}-1\right) \rho\right) . \quad[\mathrm{J} 4,6.2(1)$ p. 190].

One also can see (vii) as the same way of proving Prop. 2.6.

(viii) The module $V_{\mathfrak{t}}\left(\left(p^{a}-1\right) \rho\right)$ is irreducible. And as a $\tilde{\mathfrak{u}}_{a}$-module, $V_{\mathfrak{t}}\left(\left(p^{a}-1\right) \rho\right)$ is isomorphic to $\tilde{Z}_{\mathfrak{t}, a}\left(\left(p^{a}-1\right) \rho\right)$.

Proof. Use (iii) and (vii).

By (vii) we get

(ix) Assume that $\lambda \in \mathbf{Z}_{+}^{n}$ is $p^{a}$-restricted. Then there exists a nozero element $\mathfrak{y}_{\lambda}^{\prime}$ in $\mathfrak{u}_{a}^{-}$(unique up to a scalar) of degree $\lambda-w_{0} \lambda$ such that $\mathfrak{y}_{\lambda}^{\prime} \tilde{1}_{\mu, a}$ is primitive in $\tilde{Z}_{\mathfrak{f}, a}\left(\left(p^{a}-1\right) \rho+\lambda\right)$, where $\mu=\left(p^{a}-1\right) \rho+\lambda$. Necessarily, $\mathfrak{y}_{\lambda}^{\prime} \tilde{1}_{\mu, a}$ generates the unique irreducible submodule of $\tilde{Z}_{\mathfrak{i}, a}\left(\left(p^{a}-1\right) \rho+\lambda\right)$.

Using $6.1(\mathrm{v})$, in the same way as the proof of Theorem 3.7 (ii) we get (x) Assume that $\lambda$ is $p^{a}$-restricted. Then $\mathfrak{y}_{\lambda}^{\prime} v_{\mu}$ is primitive in $V_{\mathbf{t}}(\mu)$, where $\mu=\left(p^{a}-1\right) \rho+\lambda$ and $\mathfrak{y}_{\lambda}^{\prime} v_{\mu}$ generates an irreducible submodule of $V_{\mathrm{t}}(\mu)$, which is isomorphic to $L_{\mathrm{f}}\left(\left(p^{a}-1\right) \rho+w_{0} \lambda\right)$. (Cf. [J4, Section 6.3, p. 191].)

Assume that $\lambda \in \mathbf{Z}_{+}^{n}$ is $p^{a}$-restricted and $b \geq a$. Let $M:=L_{\mathfrak{f}}\left(p^{a}\left(p^{b-a}-1\right) \rho\right)$ $\otimes V_{t}\left(\left(p^{a}-1\right) \rho+\lambda\right)$ and $m:=\bar{v}_{p^{a}\left(p^{b-a}-1\right) \rho} \otimes v_{\left(p^{a}-1\right) \rho+\lambda}$.

(xi) Keep the notation above: Regarding $M$ as a $\tilde{\mathfrak{u}}_{b}$-module, we have a unique $\tilde{\mathfrak{u}}_{b}$-homomorphism $\tilde{Z}_{\mathfrak{t}, b}\left(\left(p^{b}-1\right) \rho+\lambda\right) \rightarrow M$ which carries $\tilde{1}_{\left(p^{b}-1\right) \rho+\lambda, b}$ to $m$. We claim the homomorphism is injective.

Proof. By (viii) and 6.1 (iii), the elements $f_{\beta_{r}}^{\left(k_{r} p^{a}\right)} f_{\beta_{r-1}}^{\left(k_{r}-1 p^{a}\right)} \cdots f_{\beta_{1}}^{\left(k_{1} p^{a}\right)} \bar{v}_{p^{a}\left(p^{b-a}-1\right) \rho}$ $\left(0 \leq k_{r}, \ldots, k_{1} \leq p^{b-a}-1\right)$ form a basis of $L_{\mathfrak{t}}\left(p^{a}\left(p^{b-a}-1\right) \rho\right)$. Since $\mathfrak{I}_{\left(p^{a}-1\right) \rho+\lambda}^{-}$ $\subseteq \mathfrak{I}_{\left(p^{a}-1\right) \rho}^{-}$, by (vii) and (viii), the elements $f_{\beta_{1}}^{\left(k_{1}\right)} f_{\beta_{2}}^{\left(k_{2}\right)} \ldots f_{\beta_{r}}^{\left(k_{r}\right)} v_{\left(p^{a}-1\right) \rho+\lambda}\left(0 \leq k_{1}, \ldots\right.$, $\left.k_{r} \leq p^{a}-1\right)$ are linearly independent in $V_{\mathrm{f}}\left(\left(p^{a}-1\right) \rho+\lambda\right)$. Combining these and using (iv) and 6.1 (iv), we see

$$
f_{\left(p^{b}-1\right) \rho} m=f_{p^{a}\left(p^{b-a}-1\right) \rho}^{\prime}\left(\bar{v}_{p^{a}\left(p^{b-a}-1\right) \rho} \otimes f_{\left(p^{a}-1\right) \rho} v_{\left(p^{a}-1\right) \rho+\lambda}\right) \neq 0 .
$$

By (vii) we see the homomorphism is injective.

Since $\mathfrak{y}_{\lambda}^{\prime} m=\bar{v}_{p^{a}\left(p^{b-a}-1\right) \rho} \otimes \mathfrak{y}_{\lambda}^{\prime} v_{\left(p^{a}-1\right) \rho+\lambda}$ is primitive in $M$ (see $\left.(\mathrm{x})\right)$ and $\mathfrak{y}_{\lambda}^{\prime} \in \mathfrak{u}_{a}^{-} \subseteq \mathfrak{u}_{b}^{-}$, using (xi) we see $\mathfrak{y}_{\lambda}^{\prime} \tilde{1}_{\left(p^{b}-1\right) \rho+\lambda, b}$ is primitive in $\tilde{Z}_{\mathfrak{t}, b}\left(\left(p^{b}-1\right) \rho+\lambda\right)$. Applying (ix) we get

(xii) Assume that $\lambda \in \mathbf{Z}_{+}^{n}$ is $p^{a}$-restricted and $b \geq a$. Let $\mathfrak{y}_{\lambda}^{\prime}$ be as in (ix). Then $\mathfrak{y}_{\lambda}^{\prime} \tilde{1}_{\left(p^{b}-1\right) \rho+\lambda, b}$ is primitive in $\tilde{Z}_{\mathfrak{f}, b}\left(\left(p^{b}-1\right) \rho+\lambda\right)$ and generates the unique irreducible submodule of $\tilde{Z}_{\mathfrak{t}, b}\left(\left(p^{b}-1\right) \rho+\lambda\right)$ which is isomorphic to $\tilde{L}_{\mathfrak{t}, b}\left(\left(p^{b}-1\right) \rho+w_{0} \lambda\right)$.

Applying (x) we get

(xiii) Assume that $\lambda \in \mathbf{Z}_{+}^{n}$ is $p^{a}$-restricted and $b \geq a$. Let $\mathfrak{y}_{\lambda}^{\prime}$ be as in (ix). Then $\mathfrak{y}_{\lambda}^{\prime} v_{\left(p^{b}-1\right) \rho+\lambda}$ is primitive in $V_{\mathfrak{t}}\left(\left(p^{b}-1\right) \rho+\lambda\right)$ and generates an irreducible submodule of $V_{\mathfrak{t}}\left(\left(p^{b}-1\right) \rho+\lambda\right)$ with highest weight $\left(p^{b}-1\right) \rho+w_{0} \lambda$.

Remark. Let $\lambda$ be $p^{a}$-restricted. According to 3.3 (viii), $M^{\prime}:=V_{\mathbf{f}}\left(\left(p^{a}-1\right) \rho\right.$ ) $\otimes V_{\mathfrak{t}}(\lambda)$ has a filtration of Weyl modules. So $V_{\mathfrak{t}}\left(\left(p^{a}-1\right) \rho+\lambda\right)$ is isomorphic to 
a submodule of $M^{\prime}$ (cf. Lemma 3.6 (i)). It is well known that as a $\tilde{\mathfrak{u}}_{a}$-module, $M^{\prime}$ is projective and injective. Therefore $V_{t}\left(\left(p^{a}-1\right) \rho+\lambda\right)$ has a unique irreducible $\mathfrak{U}_{\mathfrak{t}}$-module if as a $\tilde{\mathfrak{u}}_{a}$-module $V_{\mathfrak{t}}\left(\left(p^{a}-1\right) \rho+\lambda\right)$ is indecomposable.

6.3. Given $\lambda \in \mathbb{Z}_{+}^{n}, w \in W$, define the monomials $\mathfrak{r}_{\lambda, w}, \mathfrak{r}_{\lambda, w}^{\prime}, \mathfrak{r}_{\lambda}, \mathfrak{r}_{\lambda}^{\prime}$ of $f_{i}^{(k)}$ $(i=1,2, \ldots, n, k \geq 0)$ in the same way as 1.4. Depending on the contexts, the monomials will be regarded as elements in $\mathfrak{U}_{\mathfrak{t}}$ or elements in $\mathfrak{U}$. We state the analogues of a few results in Section 4 and Section 5. The letters $a, b$ will stand for positive integers.

Lemma 6.4. Assume that $\lambda \in \mathbb{Z}_{+}^{n}$ is $p^{a}$-restricted and $b>6 a\left|R^{+}\right|$. Let $w \in W$ and $\mu=\left(p^{b}-1\right) \rho+\lambda$. Then

(i) In $V_{\mathbf{i}}(\mu)$ we have $\mathfrak{r}_{\lambda, w}^{\prime} v_{\mu} \neq 0$.

(ii) If $k \geq 1$, then in $V_{\mathrm{i}}(\mu)$ we have $e_{i}^{(k)} \mathfrak{r}_{\lambda, w}^{\prime} u_{\mu}=0$ for $i=1,2, \ldots, n$. That is, $\mathfrak{r}_{\lambda, w}^{\prime} v_{\mu}$ is primitive in $V_{\mathbf{f}}(\mu)$. In particular we have

(iii) The element $\mathfrak{r}_{\lambda}^{\prime} v_{\mu}$ is primitive in $V_{\mathbf{i}}(\mu)$.

Proof. Part (i) is obvious (cf. Lemma 4.4 (i) and its proof). Now we prove (ii). Set $\lambda_{i}:=\left\langle\lambda, \alpha_{i}^{\vee}\right\rangle$ for $i=1,2, \ldots, n$. Use induction on $l(w)$ we see that

(a) There exist $g_{1}, g_{2}, \ldots, g_{k}$ in $\mathfrak{U}_{\mathfrak{f}}$ such that

$$
e_{i}^{(k)} \mathfrak{r}_{\lambda, w}^{\prime}=\mathfrak{r}_{\lambda, w}^{\prime} e_{i}^{(k)}+g_{1}\left(\begin{array}{c}
h_{i}+1-\lambda_{i} \\
1
\end{array}\right)+g_{2}\left(\begin{array}{c}
h_{i}+2-\lambda_{i} \\
2
\end{array}\right)+\cdots+g_{k}\left(\begin{array}{c}
h_{i}+k-\lambda_{i} \\
k
\end{array}\right) .
$$

When $b>6 a \mid R^{+}$, we must have $\left\langle u \lambda, \alpha_{j}^{\vee}\right\rangle\left\langle p^{b}\right.$ for all $u \in W$ and $j=1,2, \ldots, n$. According to the definition of $\mathfrak{r}_{\lambda, w}^{\prime}$, we may require that $g_{k^{\prime}}=0$ when $k^{\prime} \geq p^{b}$. Note that $g_{k^{\prime}}\left(\begin{array}{c}h_{1}+k^{\prime}-\lambda_{2} \\ k^{\prime}\end{array}\right) v_{\mu}=0$ if $1 \leq k^{\prime}<p^{b}$. Now according to (a) we get (ii).

The lemma is proved.

Theorem 6.5. (i) Assume that $\lambda \in \mathbb{Z}_{+}^{n}$ is $p^{a}$-restricted. Then $\mathfrak{r}_{\lambda}$ and $\mathfrak{r}_{\lambda}^{\prime}$ are elements in $\mathfrak{u}_{a}^{-}$.

(ii) $\mathfrak{r}_{\lambda}^{\prime} \tilde{1}_{\left(p^{a}-1\right) \rho+\lambda}$ is primitive in $\tilde{Z}_{\mathfrak{t}, a}\left(\left(p^{a}-1\right) \rho+\lambda\right)$ and generates the unique irreducible submodule of $\tilde{Z}_{\mathfrak{i}, a}\left(\left(p^{a}-1\right) \rho+\lambda\right)$, which is isomorphic to $\tilde{\mathbb{L}}_{\mathfrak{i}, a}\left(\left(p^{a}-1\right) \rho\right.$ $\left.+w_{0} \lambda\right)$.

Proof. Let $b>6 a\left|\mathbb{R}^{+}\right|$. Since $\mathfrak{\Im}_{\left(p^{b}-1\right) \rho+\lambda}^{-} \subseteq \mathfrak{\Im}_{\left(p^{b}-1\right) \rho}^{-}$, we see the $\tilde{\mathfrak{u}}_{b^{-}}$ homomorphism $\tilde{Z}_{\mathrm{f}, b}\left(\left(p^{b}-1\right) \rho+\lambda\right) \rightarrow \mathbb{V}_{\mathrm{f}}\left(\left(p^{b}-1\right) \rho+\lambda\right), \tilde{1}_{\left(p^{b}-1\right) \rho+\lambda, b} \rightarrow v_{\left(p^{b}-1\right) \rho+\lambda}$, is injective. By our choice of $b$ we see $\mathfrak{r}_{\lambda}^{\prime} \in \tilde{\mathfrak{u}}_{b}^{-}$. By Lemma 6.4 (iii) we see $\mathfrak{r}_{\lambda}^{\prime} \tilde{1}_{\left(p^{b}-1\right) \rho+\lambda, b}$ is primitive in $\tilde{Z}_{\mathfrak{i}, b}\left(\left(p^{b}-1\right) \rho+\lambda\right)$. Since $\mathfrak{r}_{\lambda}^{\prime}$ has degree $\lambda-w_{0} \lambda$, by 6.2 (xii), 6.2 (vii) and 6.2 (ix) (replacing $a$ by $b$ ), we see $\mathfrak{r}_{\lambda}^{\prime}=\theta \mathfrak{y}_{\lambda}^{\prime} \in \tilde{\mathfrak{u}}_{a}^{-}$for some nonzero $\theta \in \mathfrak{F}$. We have $\mathfrak{r}_{\lambda}=\mathfrak{r}_{-w_{0} \lambda}^{\prime}$, since $-w_{0} \lambda$ is also $p^{a}$-restricted, so $\mathfrak{r}_{\lambda} \in \mathfrak{u}_{a}^{-}$. (i) is proved.

(ii) follows from 6.2 (xii) and the proof of (i). 
6.6. Remark. We also can prove Theorem 6.5 (i) by using Theorem 5.2 provided that every simple component of $g$ is not of type $G_{2}$.

If $p$ is odd, choose a $p^{a}$-th primitive root $\xi$ of 1 . If $p=2$, choose a $2^{a+1}$-th primitive root $\xi$ of 1 . Let $U_{\xi}^{\prime}$ be the $Z[\xi]$-subalgebra of $U_{\xi}$ generated by the elements $E_{i}^{(k)}, F_{i}^{(k)}, K_{i}, K_{i}^{-1}$ for $i=1,2, \ldots, n, k \geq 0$. Consider the $\mathfrak{f}$-algebra $\mathscr{U}_{\mathfrak{i}}^{\prime}:=U_{\xi}^{\prime} \otimes_{\mathbf{Z}[\xi]} \mathfrak{f}$, where $\mathfrak{f}$ is regarded as a $\mathbb{Z}[\xi]$-algebra through the ring homomorphism $\mathbb{Z}[\xi] \rightarrow \mathfrak{f}, \xi \rightarrow 1$. For simplicity, the images in $\mathscr{U}_{\mathfrak{t}}^{\prime}$ of $E_{i}^{(k)}, F_{i}^{(k)}, K_{i}, K_{i}^{-1}$, etc. will be denoted by the same notations respectively.

Let $\mathscr{K}^{\prime}$ be the two-sided ideal of $\mathscr{U}_{\mathrm{i}}^{\prime}$ generated by $K_{1}-1, \ldots, K_{n}-1$. Set $\mathscr{U}_{\mathfrak{f}}:=\mathscr{U}_{\mathfrak{i}}^{\prime} / \mathscr{K}^{\prime}$. Again for simplicity, the images in $\mathscr{U}_{\mathrm{f}}$ of $E_{i}^{(k)}, F_{i}^{(k)}, K_{i}, K_{i}^{-1}$, etc. will be denoted by the same notations respectively. The following result is due to Lusztig [L3, 6.7 (d), p. 295] (cf. 1.6).

(i) There is a unique $\mathfrak{f}$-algebra isomorphism $\mathscr{U}_{\mathfrak{f}} \rightarrow \mathfrak{U}_{\mathfrak{f}}$ such that $E_{i}^{(k)}$ maps to $e_{i}^{(k)}, F_{i}^{(k)}$ maps to $f_{i}^{(k)},\left[\begin{array}{c}K_{1}, 0 \\ k\end{array}\right]$ maps to $\left(\begin{array}{c}h_{1} \\ k\end{array}\right)$, for $i=1,2, \ldots, n, k \in \mathbf{N}$.

When $g$ is of type $A_{n}, D_{n}, E_{n}$; or $B_{n}, C_{n}, F_{4}$ and $p$ is odd, Theorem 6.5 (i) is a simple consequence of (i) and Theorem 5.2. When $\mathfrak{g}$ is of type $B_{n}, C_{n}, F_{4}$ and $p=2$, one may prove Theorem 6.5 (i) by direct calculations.

Theorem 6.7. Assume that $\lambda \in \mathbf{Z}_{+}^{n}$ is $p^{a}$-restricted.

(i) Let $\mathfrak{I}_{\lambda}^{\prime}$ be the left ideal of $\mathfrak{U}_{\mathfrak{f}}$ generated by the elements $e_{i}^{(k)},\left(\begin{array}{c}h_{i} \\ k\end{array}\right)-\left(\begin{array}{c}\left\langle\lambda, \alpha_{i}^{\vee}\right\rangle \\ k\end{array}\right)$, $f_{i}^{\left(k_{2}\right)}\left(i=1,2, \ldots, n, k \geq 1, k_{i} \geq p^{a}\right)$ and elements $f \in \mathfrak{u}_{a}^{-}$such that $f \mathfrak{r}_{\left(p^{a}-1\right) \rho-\lambda}=0$, then $\mathfrak{U}_{\mathfrak{i}} / \mathfrak{I}_{\lambda}^{\prime} \simeq L_{\mathfrak{i}}(\lambda)$.

(ii) Let $\mathfrak{u}_{a}(\lambda)$ be the left ideal of $\mathfrak{u}_{a}$ generated by the elements $e_{\alpha}^{(k)},\left(\begin{array}{c}h_{l} \\ k\end{array}\right)-\left(\begin{array}{c}\left\langle\lambda, \alpha_{i}^{\vee}\right\rangle \\ k\end{array}\right)$ $\left(\alpha \in R^{+}, i=1,2, \ldots, n, 1 \leq k \leq p^{a}-1\right)$ and elements $f \in \mathfrak{u}_{a}^{-}$such that $f \mathfrak{r}_{\left(p^{a}-1\right) \rho-\lambda}$ $=0$, then $\mathfrak{u}_{a} / \mathfrak{u}_{\mathfrak{a}}(\lambda) \simeq L_{\mathfrak{f}, a}(\lambda)$.

(iii) For any $\gamma \in \mathbf{N} R^{+}$, denote $\mathfrak{u}_{a, \gamma}^{-}$the set of all elements in $\mathfrak{u}_{a}^{-}$of degree $\gamma$ and denote $\mathfrak{n}_{a}(\lambda, \gamma)$ the set $\left\{f \in \mathfrak{u}_{a, \gamma}^{-} \mid f \mathfrak{r}_{\left(p^{a}-1\right) \rho-\lambda}=0\right\}$, then

$$
\operatorname{dim} L_{\mathfrak{f}}(\lambda)_{\lambda-\gamma}=\operatorname{dim} \mathfrak{u}_{a, \gamma}^{-}-\operatorname{dim} \mathfrak{n}_{a}(\lambda, \gamma) .
$$

In particular, we have

$$
\operatorname{dim} L_{\mathfrak{f}}(\lambda)=p^{a\left|R^{+}\right|}-\operatorname{dim}\left\{f \in \mathfrak{u}_{a}^{-} \mid f \mathfrak{r}_{\left(p^{a}-1\right) \rho-\lambda}=0\right\} .
$$

Proof. Since $\mathfrak{r}_{\left(p^{a}-1\right) \rho-\lambda}=\mathfrak{r}_{\left(p^{a}-1\right) \rho+w_{0} \lambda}^{\prime}$, (ii) follows from Theorem 6.5 (ii) and 6.2 (iii). (i) and (iii) follow from (ii).

\section{§7. Questions}

7.1. Recall that $\xi$ is a root of 1 of $\operatorname{order} l \geq 3$. For $i \in[1, n], k \in \mathbf{N}$, denote $\Theta_{i, k}$ the $\mathbf{Q}(\xi)$-linear homomorphism $U_{\xi} \rightarrow U_{\xi}, x \rightarrow x F_{i}^{(k)}$. The kernel and the image of $\Theta_{i, k}$ are easily described by means of PBW Theorem. Assume that $\lambda \in \mathbb{Z}_{+}^{n}$ is I-restricted. Let $s_{i_{1}} s_{i_{2}} \cdots s_{i_{r}}$ be a reduced expression of the longest element of $W$. Set $k_{h}:=\left\langle s_{i_{h-1}} \cdots s_{i_{1}}(\kappa-\lambda), \alpha_{i_{h}}^{\vee}\right\rangle, \quad \delta_{h}:=k_{1} \alpha_{i_{1}}+\cdots+k_{h} \alpha_{i_{h}}$, 
$h=1, \ldots, r . \quad$ Recall that for any $\gamma \in \mathbf{N} R^{+}$we denote $\mathbf{u}_{\xi, \gamma}^{-}$the set of all elements in $\mathbf{u}_{\xi}^{-}$of degree $\gamma$. Given $\beta \in \mathbf{N} R^{+}$, set

$$
\begin{aligned}
& D_{0, \beta}=\operatorname{dim}_{\mathbf{Q}(\xi)} \mathbf{u}_{\xi, \beta}^{-}, \\
& D_{1, \beta}=\operatorname{dim}_{\mathbf{Q}(\xi)} \Theta_{i_{1}, k_{1}}\left(\mathbf{u}_{\xi, \beta}^{-}\right), \\
& D_{2, \beta}=\min \left\{D_{1, \beta}, \operatorname{dim}_{\mathbf{Q}(\xi)} \Theta_{i_{2}, k_{2}}\left(\mathbf{u}_{\xi, \beta}^{-}-\delta_{1}\right)\right\}, \\
& \ldots \ldots \\
& D_{h, \beta}=\min \left\{D_{h-1, \beta}, \operatorname{dim}_{\mathbf{Q}(\xi)} \Theta_{i_{h}, k_{h}}\left(\mathbf{u}_{\xi, \beta+\delta_{h-1}}^{-}\right)\right\}, \\
& \ldots \ldots \\
& D_{r, \beta}=\min \left\{D_{r-1, \beta}, \operatorname{dim}_{\mathbf{Q}(\xi)} \Theta_{i_{r}, k_{r}}\left(\mathbf{u}_{\xi, \beta+\delta_{r-1}}^{-}\right)\right\} .
\end{aligned}
$$

Conjecture A. The number $D_{r, \beta}$ is independent of the choice of the reduced expression of $w_{0}$ and $\operatorname{dim}_{\mathbf{Q}(\xi)} L_{\xi}(\lambda)_{\lambda-\beta}=D_{r, \beta}$.

7.2. For $i \in[1, n], k \in \mathbb{N}$, denote $\theta_{i, k}$ the $\mathfrak{f}$-linear homomorphism $\mathfrak{U}_{\mathfrak{f}} \rightarrow \mathfrak{U}_{\mathfrak{f}}$, $x \rightarrow x f_{i}^{(k)}$. The kernel and the image of $\theta_{i, k}$ are easily described by means of PBW Theorem. Assume that $\lambda \in \mathbb{Z}_{+}^{n}$ is $p^{a}$-restricted. Let $s_{i_{1}} s_{i_{2}} \cdots s_{i_{r}}$ be a reduced expression of the longest element of $W$. Set $k_{h}:=\left\langle s_{i_{h-1}} \cdots s_{i_{1}}\left(\left(p^{a}-1\right) \rho\right.\right.$ $\left.-\lambda), \alpha_{i_{h}}^{v}\right\rangle, \delta_{h}:=k_{1} \alpha_{i_{1}}+\cdots+k_{h} \alpha_{i_{h}}, h=1, \ldots, r$. Recall that for any $\gamma \in \mathbb{N}^{+}$ we denote $\mathfrak{u}_{a, \gamma}^{-}$the set of all elements in $\mathfrak{u}_{a}^{-}$of degree $\gamma$. Given $\beta \in \mathbb{N} R^{+}$, set

$$
\begin{aligned}
& \mathfrak{d}_{0, \beta}=\operatorname{dim} \mathfrak{u}_{\xi, \beta}^{-}, \\
& \mathfrak{d}_{1, \beta}=\operatorname{dim} \theta_{i_{1}, k_{1}}\left(\mathfrak{u}_{\xi, \beta}^{-}\right), \\
& \mathfrak{d}_{2, \beta}=\min \left\{\mathfrak{d}_{1, \beta}, \operatorname{dim} \theta_{i_{2}, k_{2}}\left(\mathfrak{u}_{\xi, \beta}^{-}+\delta_{1}\right)\right\}, \\
& \ldots \ldots \\
& \mathfrak{D}_{h, \beta}=\min \left\{\mathfrak{d}_{h-1, \beta}, \operatorname{dim} \theta_{i_{h}, k_{h}}\left(\mathfrak{u}_{\xi, \beta+\delta_{h-1}}^{-}\right)\right\}, \\
& \ldots \ldots \\
& \mathfrak{b}_{r, \beta}=\min \left\{\mathfrak{d}_{r-1, \beta}, \operatorname{dim} \theta_{i_{r}, k_{r}}\left(\mathfrak{u}_{\xi, \beta+\delta_{r-1}}^{-}\right)\right\} .
\end{aligned}
$$

Conjecture $\mathbb{B}$. The number $\mathfrak{D}_{r, \beta}$ is independent of the choice of the reduced expression of $w_{0}$ and $\operatorname{dim} L_{\mathfrak{i}, a}(\lambda)_{\lambda-\beta}=\mathfrak{D}_{r, \beta}$ provided that $p \geq$ the Coxeter number of the root system $R$ associated to $\mathrm{g}$.

7.3. Let $\phi_{l}$ be the $l$-th cyclomatic polynomial (i.e. the minimal polynomial of $\xi$ ). Denote by $\mathscr{A}$ the localization of $\mathbb{Q}\left[v, v^{-1}\right]$ at its prime ideal generated by $\phi_{l}$. Let $U_{\mathscr{A}}$ be the $\mathscr{A}$-subalgebra of $U$ generated by the elements $E_{i}^{(a)}, F_{i}^{(a)}, K_{i}, K_{i}^{-1}$ for $i=1,2, \ldots, n, a \geq 0$ and let $U_{\mathscr{A}}^{b}$ be the $\mathscr{A}$-subalgebra of $U_{\mathscr{A}}$ generated by the elements $F_{i}^{(a)}, K_{i}, K_{i}^{-1},\left[\begin{array}{c}K_{i}, c \\ a\end{array}\right]$ for $i=1,2, \ldots, n, c \in \mathbb{Z}$, 
$a \in \mathbf{N}$. Define the category $\mathscr{C}$ (resp. $\mathscr{C}^{b}$ ) of $U_{\mathscr{A}}$-modules (resp. $U_{\mathscr{A}}^{b}$-modules) as in [APW, 2.2, p.17]. Then define the induction functor $H^{0}: \mathscr{C}^{b} \rightarrow \mathscr{C}$ as in [APW, 2.8, p. 19]. For each $k \in \mathbf{N}$ we then have a derived functor $H^{k}: \mathscr{C}^{b} \rightarrow \mathscr{C}$.

Given $\lambda=\left(\lambda_{1}, \lambda_{2}, \ldots, \lambda_{n}\right) \in \mathbb{Z}^{n}$, let $F_{i}^{(a)}$ acts on $\mathscr{A}$ by scalar zero and let $K_{i},\left[\begin{array}{c}K_{i}, c \\ a\end{array}\right]$ act on $\mathscr{A}$ by scalar $v^{d_{i} \lambda_{2}},\left[\begin{array}{c}\lambda_{i}+c \\ a\end{array}\right]_{d_{i}}$ respectively, $i=1,2, \ldots, n$, $c \in \mathbb{Z}, a \geq 1$. This defines a $U_{\mathscr{A}}^{b}$-module structure on $\mathscr{A}$. We denote the $U_{\mathscr{A}}^{b}$-module by $\mathscr{A}_{\lambda}$. We shall simply write $H^{k}(\lambda)$ for $H^{k}\left(\mathscr{A}_{\lambda}\right)$.

Recall that $U_{v}=U$. We drop the index $v$ and the index 1 in all notations involving them. So $V(\lambda)$ will stand for $V_{v}(\lambda)$. Let $\lambda, \mu \in \mathbb{Z}_{+}^{n}$. Assume that $\lambda \in \mathbb{Z}_{+}^{n}$ is 1-restricted. Given $w \in W$, set

$$
H_{w}(\mathbf{l} \mu+\lambda):=\left\{y v_{\mathbf{l} \mu+\lambda} \mid y \in U \text { and } y x_{\kappa-\lambda, w} \in U_{\mathscr{A}}\right\} .
$$

Then $H_{w}(l \mu+\lambda)$ is a free $\mathscr{A}$-submodule of $V(\mathbf{l} \mu+\lambda)$.

Conjecture C. The $U_{\mathscr{A}}$-module $H_{w}(1 \mu+\lambda)$ is the free part of the cohomology group $H^{l\left(w^{-1} w_{0}\right)}\left(w^{-1} w_{0}(l \mu+\lambda+\rho)-\rho\right)$.

7.4. Keep the notations in Section 6. Denote by $A$ the localization of $\mathbf{Z}$ at its prime ideal generated by $p$. Let $\mathfrak{U}_{A}$ be the $A$-subalgebra of $\mathfrak{U}$ generated by the elements $e_{i}^{(k)}, f_{i}^{(k)}$ for $i=1,2, \ldots, n, k \geq 0$ and let $\mathfrak{U}_{A}^{b}$ be the $A$-subalgebra of $\mathfrak{U}_{A}$ generated by the elements $f_{i}^{(k)},\left(\begin{array}{c}h_{2}+c \\ k\end{array}\right)$ for $i=1,2, \ldots, n, c \in \mathbf{Z}, k \in \mathbf{N}$. Define the category $\mathfrak{C}$ (resp. $\mathfrak{C}^{b}$ ) of $\mathfrak{U}_{A}$-modules (resp. $\mathfrak{U}_{A}^{b}$-modules) in a similar way of $[\mathrm{APW}, 2.2, \mathrm{p} .17]$. Then define the induction functor $\mathscr{H}^{0}: \mathbb{C}^{b} \rightarrow \mathbb{C}$ as in $[\mathrm{APW}, 2.8$, p. 19]. For each $k \in \mathbf{N}$ we then have a derived functor $\mathscr{H}^{k}: \mathbb{C}^{b} \rightarrow \mathfrak{C}$.

Given $\lambda=\left(\lambda_{1}, \lambda_{2}, \ldots, \lambda_{n}\right) \in \mathbb{Z}^{n}$, let $f_{i}^{(k)}$ acts on $A$ by scalar zero and let $\left(\begin{array}{c}h_{1}+c \\ k\end{array}\right)$ acts on $A$ by scalar $\left(\begin{array}{c}\lambda_{3}+c \\ k\end{array}\right)$ for $i=1,2, \ldots, n, c \in \mathbb{Z}, k \geq 1$. This defines a $\mathfrak{U}_{A}^{b}$-module structure on $A$. We denote the $\mathfrak{U}_{A}^{b}$-module by $A_{\lambda}$. We shall simply write $\mathscr{H}^{k}(\lambda)$ for $\mathscr{H}^{k}\left(A_{\lambda}\right)$.

For every $\lambda \in \mathbb{Z}_{+}^{n}$, denote $M(\lambda)$ an irreducible $\mathfrak{U}$-module of highest weight $\lambda$. Let $m_{\lambda}$ be a nonzero element in $M(\lambda)$ of weight $\lambda$. Assume that $\lambda \in \mathbf{Z}_{+}^{n}$ is $p^{a}$-restricted. Given $w \in W$, set

$$
\mathscr{H}_{w}(\lambda):=\left\{y m_{\lambda} \mid y \in \mathfrak{U} \text { and } y \mathfrak{r}_{\left(p^{a}-1\right) \rho-\lambda, w} \in \mathfrak{U}_{A}\right\} .
$$

Then $\mathscr{H}_{w}(\lambda)$ is a free $A$-submodule of $M(\lambda)$.

Conjecture D. (i) The $\mathfrak{U}_{A}$-module $\mathscr{H}_{w}(\lambda)$ is well defined and is the free part of the cohomology group $\mathscr{H}^{l\left(w^{-1} w_{0}\right)}\left(w^{-1} w_{0}(\lambda+\rho)-\rho\right)$.

(ii) The module $\mathscr{H}^{l(w)}(w(\lambda+\rho)-\rho)$ is isomorphic to the cohomology group $H^{l(w)}\left(G_{A} / B_{A}, w(\lambda+\rho)-\rho\right)$ defined in $[A$, Section 2, p. 501] (which has a natural 


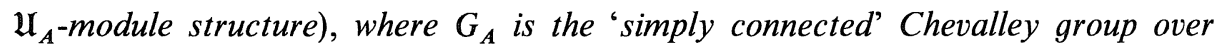
$A$ and associated to $\mathrm{g}$ and $B_{A}$ is a suitable 'Borel subgroup' of $G_{A}$.

7.5. Keep the notations in 7.3. Let $s_{i_{k}} \cdots s_{i_{2}} s_{i_{1}}$ be a reduced expression of $w$. For $h=1, \ldots, k$, set $a_{h}:=\left\langle s_{i_{h-1}} s_{i_{h-2}} \cdots s_{i_{1}} \lambda, \alpha_{i_{h}}^{\vee}\right\rangle, v_{h}:=\left\langle\mathbf{l} \mu+\kappa, \alpha_{i_{h}}^{\vee}\right\rangle+a_{h}$, $d_{h}^{\prime}=d_{i_{h}}$. Then define

$$
a_{\lambda, w}:=\left[\begin{array}{l}
v_{1} \\
a_{1}
\end{array}\right]_{d_{1}^{\prime}}\left[\begin{array}{l}
v_{2} \\
a_{2}
\end{array}\right]_{d_{2}^{\prime}} \ldots\left[\begin{array}{c}
v_{k} \\
a_{k}
\end{array}\right]_{d_{k}^{\prime}} .
$$

Conjecture E. As $U_{\mathscr{A}}$-modules, $U_{\mathscr{A}} x_{\lambda, w}^{\prime} v_{\mathbf{l} \mu+\kappa+\lambda} / a_{\lambda, w}$ is isomorphic to $H_{w}(\mathbf{l} \mu+\kappa+\lambda)$.

7.6. Keep the notations in 7.4. Let $s_{i_{k}} \cdots s_{i_{2}} s_{i_{1}}$ be a reduced expression of $w$. For $h=1, \ldots, k$, set $a_{h}:=\left\langle s_{i_{h-1}} s_{i_{h-2}} \cdots s_{i_{1}} \lambda, \alpha_{i_{h}}^{\vee}\right\rangle, v_{h}:=p^{a}-1+a_{h}$. And define

$$
\tilde{a}_{\lambda, w}:=\left(\begin{array}{c}
v_{1} \\
a_{1}
\end{array}\right)\left(\begin{array}{l}
v_{2} \\
a_{2}
\end{array}\right) \cdots\left(\begin{array}{c}
v_{k} \\
a_{k}
\end{array}\right) .
$$

Conjecture F. As $\mathfrak{U}_{A}$-modules, $\mathfrak{U}_{A} \mathfrak{r}_{\lambda, w}^{\prime} v_{\mu} / \tilde{a}_{\lambda, w}$ is isomorphic to $\mathscr{H}_{w}(\mu)$, where $\mu=\left(p^{a}-1\right) \rho+\lambda$.

7.7. Keep the notations in 7.3. Let $\Omega: U_{\mathscr{A}} \rightarrow U_{\mathscr{A}}^{o p p}$ be the Q-algebra homomorphism defined by (cf. [L4, 1.1 (d1), p.91])

$$
\Omega E_{i}^{(a)}=F_{i}^{(a)}, \Omega F_{i}^{(a)}=E_{i}^{(a)}, \Omega K_{i}=K_{i}^{-1}, \Omega v=v^{-1} .
$$

Given $\lambda \in \mathbb{Z}_{+}^{n}$, we define $V_{\mathscr{A}}(\lambda):=U_{\mathscr{A}} v_{\lambda}$. There exists a unique $\mathbf{Q}$-bilinear form ( , ): $V_{\mathscr{A}}(\lambda) \times V_{\mathscr{A}}(\lambda) \rightarrow \mathscr{A}$ such that (cf. [CK, 1.9, p. 482])

(a) $\left(\varphi u, u^{\prime}\right)=\varphi\left(u, u^{\prime}\right),\left(u, \varphi u^{\prime}\right)=\bar{\varphi}\left(u, u^{\prime}\right)$,

(b) $\left(u, u^{\prime}\right)=\overline{\left(u^{\prime}, u\right)}$,

(c) $\left(v_{\lambda}, v_{\lambda}\right)=1,\left(x u, u^{\prime}\right)=\left(u, \Omega(x) u^{\prime}\right)$,

where $\varphi=\varphi(v) \in \mathscr{A}$ and $\bar{\varphi}=\varphi\left(v^{-1}\right)$ (that is, - denotes the $\mathbf{Q}$-algebra homomorphism $\mathscr{A} \rightarrow \mathscr{A}, v \rightarrow v^{-1}$ ); $u, u^{\prime}$ are elements in $V_{\mathscr{A}}(\lambda)$ and $x \in U_{\mathscr{A}}$.

Assume that $\lambda$ is l-restricted and $\mu \in \mathbb{Z}_{+}^{n}$. For each integer $k \in \mathbb{N}$, set $V_{\mathscr{A}}(\mathbf{l} \mu+\lambda)_{k}:=\left\{u \in V_{\mathscr{A}}(\mathbf{l} \mu+\lambda) \mid\left(u, u^{\prime}\right) \in \phi_{l}^{k} \mathscr{A}\right.$ for all elements $u^{\prime}$ in $\left.V_{\mathscr{A}}(\mathbf{l} \mu+\lambda)\right\}$,

$$
V_{\mathscr{A}}(\mathrm{l} \mu+\lambda)_{k}^{\prime}:=\left\{y v_{\mathbf{1} \mu+\lambda} \mid y \in U_{\mathscr{A}} \text { and } y x_{\kappa-\lambda} \in \phi_{l}^{k} U_{\mathscr{A}}\right\} \text {. }
$$

Assertion. We have $V_{\mathscr{A}}(\mathbf{l} \mu+\lambda)_{q+1}^{\prime} \subseteq \phi_{l} V_{\mathscr{A}}(\mathbb{l} \mu+\lambda)$, where $q=\#\left\{\alpha \in R^{+} \mid\left\langle\kappa-\lambda, \alpha^{v}\right\rangle\right.$ is not divisible by $\left.l_{\alpha}\right\}$. In particular we have $V_{\mathscr{A}}(\mathbb{l} \mu+\lambda)_{\mathbf{r}+1}^{\prime} \subseteq \phi_{l} V_{\mathscr{A}}(\mathbb{l} \mu+\lambda)$ (recall that $\left.r=\left|R^{+}\right|\right)$.

Proof. For each $x \in U_{\mathscr{A}}, x F_{i}^{(k)}$ is not in $\phi_{l} U_{\mathscr{A}}$ if $x \notin \phi_{l} U_{\mathscr{A}}$ and $k$ is divisible 
by $l_{i}$. Now the assertion follows from Prop. 41.1.7 in [L7, p. 326] and the definition of $x_{\kappa-\lambda}$.

Conjecture G. $\quad V_{\mathscr{A}}(\mathbf{l} \mu+\lambda)_{k}=V_{\mathscr{A}}(\mathbf{l} \mu+\lambda)_{k}^{\prime}$ for $k=0,1,2, \ldots, r, r+1$.

7.8. Keep the notations in 7.4. Let $\omega: \mathfrak{U}_{A} \rightarrow \mathfrak{U}_{A}^{\text {opp }}$ be the $A$-algebra homomorphism defined by

$$
\omega e_{\alpha}^{(k)}=f_{\alpha}^{(k)}, \quad \omega f_{\alpha}^{(k)}=e_{\alpha}^{(k)} .
$$

For each $\lambda \in \mathbf{Z}_{+}^{n}$, we set $M_{A}(\lambda):=\mathfrak{U}_{A} m_{\lambda}$. There exists a unique $A$-bilinear form (, ): $M_{A}(\lambda) \times M_{A}(\lambda) \rightarrow A$ such that (cf. [J2, Section 2, p. 56])

(a) $\left(\theta m, m^{\prime}\right)=\left(m, \theta m^{\prime}\right)=\theta\left(m, m^{\prime}\right)$,

(b) $\left(m, m^{\prime}\right)=\left(m^{\prime}, m\right)$,

(c) $\left(m_{\lambda}, m_{\lambda}\right)=1,\left(g m, m^{\prime}\right)=\left(m, \omega(g) m^{\prime}\right)$,

where $\theta \in A, m, m^{\prime}$ are elements in $M_{A}(\lambda)$ and $g \in \mathfrak{U}_{A}$.

Assume that $\lambda$ is $p^{a}$-restricted. Following Jantzen [J2, Lemma 3, p. 56], for each integer $k \in \mathbf{N}$, set

$$
\begin{aligned}
& M_{A}(\lambda)_{k}:=\left\{m \in M_{A}(\lambda) \mid\left(m, m^{\prime}\right) \in p^{k} A \text { for all elements } m^{\prime} \text { in } M_{A}(\lambda)\right\}, \\
& M_{A}(\lambda)_{k}^{\prime}:=\left\{g m_{\lambda} \mid g \in \mathfrak{U}_{A} \text { and } g^{\mathfrak{r}}\left(p^{a-1) \rho-\lambda} \in p^{k} \mathfrak{U}_{A}\right\} .\right.
\end{aligned}
$$

Conjecture H. $M_{A}(\lambda)_{k}=M_{A}(\lambda)_{k}^{\prime}$ for every $k \in \mathbf{N}$.

7.9. Recall that in $U^{-}$a monomial of $F_{i}^{(k)}(i=1,2, \ldots, n, k \geq 0)$ is called to be tight (resp. semi-tight) [L8, Section 1, p. 108] if the monomial is an element of the canonical basis of $U^{-}$(resp. a $\mathbf{Z}$-linear combination of elements in the canonical basis of $U^{-}$).

It was hoped that for each $\lambda \in \mathbb{Z}_{+}^{n}$ and $w \in W$, the monomials $x_{\lambda, w}, x_{\lambda, w}^{\prime} \in U^{-}$are tight. This is true for type $A_{1}, A_{2}, A_{3}, B_{2}$ and $A_{4}$ (see [L5, 3.4; L8, Prop. 13; L6, 12.8, p. 64; X2]). But in general this is not true. For example, for type $G_{2}$, let $\lambda=(1,0)$, then $x_{\lambda}$ is semi-tight but not tight (see [X2]). I donot know whether all $x_{\lambda, w}, x_{\lambda, w}^{\prime}$ are semi-tight, or equivalently all $x_{\lambda}^{\prime}$ are semi-tight.

We may express the elements $x_{\lambda}$ as $\mathbf{Q}(v)$-linear combinations of various Poincare-Birkhoff-Witt Bases. It is rather difficult to see relations between the coefficients and Kazhdan-Lusztig polynomials for affine Weyl groups, even for type $A_{2}$.

7.10. Recall that in 2.1 we have defined the integer $l_{\alpha}$ for each $\alpha \in R^{+}$. Assume that $\mathfrak{g}$ is simple. In $\mathbb{R}^{n}$, consider the hyperplanes

$$
H_{\alpha, k}:=\left\{e \in \mathbb{R}^{n} \mid\left\langle e+\rho, \alpha^{\vee}\right\rangle=k l_{\alpha}\right\}, \quad \alpha \in R^{+}, k \in \mathbb{Z} .
$$

Denote by $s_{\alpha, k}$ the corresponding reflections of $\mathbb{R}^{n}$, that is 


$$
s_{\alpha, k}(e)=e-\left(\left\langle e+\rho, \alpha^{\vee}\right\rangle-k l_{\alpha}\right) \alpha, \quad e \in \mathbb{R}^{n} .
$$

These reflections generate an affine Weyl group $W_{1}$, which is the affine Weyl group associated to the Cartan matrix $\left(a_{i j}\right)$ when $l_{1}=\cdots=l_{n}$, the affine Weyl group associated to the transpose matrix of the Cartan matrix $\left(a_{i j}\right)$ when $l_{i} \neq l_{j}$ for some $i, j$.

Conjecture I. The Conjecture 8.2 in $[\mathrm{L} 2, \mathrm{p} .75]$ is true in terms of $W_{1}$ and $U_{\xi}$

7.11. It would be interesting to describe clearly the injective hull (or projective cover) in $\mathscr{C}$ of $L_{\xi}(\lambda)\left(\lambda \in \mathbb{Z}_{+}^{n}\right)$.

It is known that the category $\tilde{\mathfrak{C}}_{a}$ of finite dimensional $\tilde{\mathfrak{u}}_{a}$-modules has enough injective and projective objects. Question: describe clearly the injective hulls (or projective covers) in $\widetilde{\mathfrak{C}}_{a}$ of irreducible $\tilde{\mathfrak{u}}_{a}$-modules of finite dimension.

7.12. We give some indication of evidence and motivations for the conjectures above. All conjectures are true for type $A_{1}$. Conjectures $\mathrm{C}$ and $\mathrm{G}$ are true for $\lambda=\kappa$, Conjectures $\mathrm{D}$ and $\mathrm{H}$ are true for $\lambda=\left(p^{a}-1\right) \rho$, Conjectures $\mathbb{E}$ and $\mathrm{F}$ are true for $\lambda=0$.

For an irreducible $U$-module $L$ of finite dimension, one may compute the character ch $(L)$ of $L$ through Weyl's character formula. In [L5, Theorem 8.13 ; L6, 12.5, p. 63], an effective algorithm for computing $\operatorname{ch}(L)$ has been established (except for type $G_{2}$ ). It would be interesting to find an effective algorithm for computing the character $\operatorname{ch}\left(L_{\xi}\right)$ (resp. ch $\left(L_{\mathrm{f}}\right)$ ) of an irreducible $U_{\xi}$-module $L_{\xi}$ (resp. $\mathfrak{U}_{\mathfrak{f}}$-module) of finite dimension. For types $A_{2}, B_{2}$, the author also checked some cases for Conjectures A and B. In Conjecture B there is a restriction on $p$, which is based on the following example due to Andersen and Jantzen.

Assume that $l$ is a prime number $\geq 3$ and $0<a<l-1$. Let $\lambda=$ $(a, l-1, l-1, \ldots, l-1, l-a-2)$ and $\lambda^{\prime}=(l-a-2, a, l-1, \ldots, l-1, l-a-2, a)$ be elements in $\mathbb{Z}_{+}^{l+2}$. If char $\mathfrak{f}=l$, then for type $A_{l+2}$ one has $\operatorname{ch} L_{\xi}(\lambda)=\operatorname{ch} V_{\xi}(\lambda)$ and $\operatorname{ch} L_{\mathfrak{t}}(\lambda)=\operatorname{ch} V_{\mathfrak{t}}(\lambda)-\operatorname{ch} V_{\mathfrak{t}}\left(\lambda^{\prime}\right)$.

The $U_{\xi}$-module $V_{\xi}\left(\mathbb{\mu} \mu+2 \kappa+w_{0} \lambda\right)$ has a unique irreducible submodule which is isomorphic to $L_{\xi}(1 \mu+\lambda)$ and is generated by $x_{\kappa-\lambda} v_{1 \mu+2 \kappa+w_{0} \lambda}$ (see Theorems 3.7 and 4.2). From this one should be able to show $H_{w_{0}}(1 \mu+\lambda)$ $\otimes_{\mathscr{A}} \mathbf{Q}(\xi)$ has a unique irreducible submodule which is isomorphic to $L_{\xi}(\mathbb{l} \mu+\lambda)$. Thus $H_{w_{0}}(\mathbb{l} \mu+\lambda)$ is isomorphic to $H^{0}(\mathbb{l} \mu+\lambda)$. Of course we should have $H_{e}(l \mu+\lambda) \simeq H^{l\left(w_{0}\right)}\left(w_{0}(l \mu+\lambda+\rho)-\rho\right)$ (this is true when $l$ is a prime number $>3$, see [APW, Theorem 7.3, p.39]). Another evidence is the comparison between the natural homomorphisms

$$
H_{w}(\mathbb{l} \mu+\lambda) \rightarrow H_{w w^{\prime}}(\mathbb{l} \mu+\lambda),
$$


$H^{l\left(w^{-1} w_{0}\right)}\left(w^{-1} w_{0}(1 \mu+\lambda+\rho)-\rho\right) \rightarrow H^{l\left(w^{\prime-1} w^{-1} w_{0}\right)}\left(w^{-1} w^{-1} w_{0}(1 \mu+\lambda+\rho)-\rho\right)$, where $w, w^{\prime} \in W$ and $l\left(w w^{\prime}\right)=l(w)+l\left(w^{\prime}\right)$. For Conjecture $\mathrm{D}$, the motivation is similar.

Conjectures $\mathrm{E}$ and $\mathrm{F}$ are true if $l(w) \leq 1$. I hope that it is not difficult to prove them for $w=w_{0}$.

It should not be difficult to prove that if $y v_{\mathbf{l} \mu+\lambda} \neq 0$ in $V(\mathbf{l} \mu+\lambda)$, then $y x_{\kappa-\lambda} v_{\mathbf{l} \mu+2 \kappa+w_{0} \lambda} \neq 0$ in $V\left(\mathbf{l} \mu+2 \kappa+w_{0} \lambda\right)$. Then one may prove Conjecture $\mathrm{G}$ for $k=1$ by using Theorems 4.2 and 3.7. The consideration for Conjecture $\mathrm{H}$ is similar.

The Conjecture I is a natural extension of Conjecture 8.2 in [L2, p. 75], which is proved (see [KL, Theorem 38.1, p.438; KT, Theorem 4.1.2]). For type $B_{2}, G_{2}$, maybe Conjecture I could be proved in a similar way as [APW, Section 11, pp. 52-54]. The linkage principal is known (see [L9, 8.3, p. 244). One may try to compute the determinant of the contrivariant form of $V_{\mathscr{A}}(\lambda)$ in a similar way as [J1, Teil II, Satz 1, p.48] (cf. [KC, Prop. 1.9, p. 483]), then get a sum formula. It would be more interesting to eliminate the restriction on $l$ in [APW].

\section{References}

[A] Andersen, H. H., Filtrations of cohomology modules for Chevalley groups, Ann. Sci. E.N.S., 16 (1983), 495-528.

[APW] Andersen, H. H., Polo, P., Wen, K., Representations of quantum algebras, Invent. Math., 104 (1991), 1-59.

[CK] De Concini, C., Kac, V. G., Representations of quantum groups at roots of 1 , in Operator algebras, Unitary Representations, Enveloping Algerbas and Invariant Theory, Actes du colloque en l'honneur de Jacques Dixmier, edited by A. Connes, M. Duflo, A. Joseph, R. Rentschler, Progr. in Math. Birkháuser, Boston · Basel - Berlin, 92 (1990), 471-506.

[HC] Harish-Chandra, Some applications of the universal enveloping algebra of a semisimple Lie algebra, Trans. Amer. Math. Soc., 70 (1951), 28-96.

[J1] Jantzen, J. C., Darstellung halbeinfacher Gruppen und zugeordnete knotravariant Formen, Bonner Math. Schriften, 67 (1973).

[J2] - Kontravariante Formen auf induzierten Darstellungen halbeinfacher Gruppen Lie-Algebren, Math. Ann., 226 (1977), 53-65.

[J3] - Über Darstellungen höherer Frobenius-Kerne halbeinfacher algebraischer Gruppen, Math. Z., 164 (1979), 271-292.

[J4] - Darstellungen halbeinfacher Gruppen und Ihrer Frobenius-Kerne, J. Reine und Angew. Math., 317 (1980), 157-199.

[K] Kashiwara, M., On crystal bases of the $q$-analogue of universal enveloping algerbas, Duke Math. J., 63 (1991), 465-516.

[KL] Kazhdan, D., Lusztig, G., Tensor structures arising from affine Lie algebras, IV, Jour. Amer. Math. Soc., 7 (1994), 383-453.

[KT] Kashiwara, M., Tanisaki, T., Kazhdan-Lusztig conjecture for affine Lie algebras with 
negative level, Duke Math. J., 77 (1995), 21-62.

[L1] Lusztig, G., Quantum deformations of certain simple modules over enveloping algebras, Adv. in Math., 70 (1988), 237-249.

[L2] — Modular representations and quantum groups, Contemp. Math. 82 (1989), $59-77$.

[L3] - Finite dimensional Hopf algebras arising from quantized universal enveloping algebras, Jour. Amer. Math. Soc., 3 (1990), 257-296.

[L4] - Q Quantum groups at roots of 1, Geom. Ded., 35 (1990), 89-114.

[L5] - Canonical bases arising from quantized enveloping algerbas, Jour. Amer. Math. Soc., 3 (1990), 447-498.

[L6] - Introduction to quantized enveloping algebras, in New Developments in Lie Theory and Their Applications, edited by Juna Tirao and Nolan Wallach, Progr. in Math. Birkháuser, Boston · Basel · Berlin, 105 (1992), 49-65.

[L7] — - Introduction to quantum groups, Progress in Mathematics 110, (Birkháuser, Boston · Basel $\cdot$ Berlin, 1993).

[L8] - Tight monomials in quantized enveloping algebras, in Quantum Deformations of Algebras and Their Representations, edited by A. Joseph and S. Schnider, Israel Mathematical Conference Proceedings, Bar-Ilan University 7 (1993), 117-132.

[L9] — Monodromic systems on affine flag manifolds, Proc. R. Soc. Lond. A, 445 (1994), 231-246.

[V] Verma, D.-N., Structure of certain induced representations of complex semisimple Lie algebras, Bull. Amer. Math. Soc. 74 (1968), 160-166.

[X1] Xi, N., Representations of finite dimensional Hopf algebras arising from quantum groups, unpublished preprint, revised version, 1990.

[X2] — Bases of quantized enveloping algebras, preprint, 1995. 